Olaf Köller, Johannes Magenheim, Heike Molitor, Uwe Pfenning, Jörg Ramseger, Mirjam Steffensky, Rudolf Tippelt, Christian Wiesmüller, Esther Winther, Bernd Wollring

\title{
Zieldimensionen für Multiplikatorinnen und Multiplikatoren früher MINT-Bildung
}

Wissenschaftliche Untersuchungen zur Arbeit der Stiftung „Haus der kleinen Forscher"

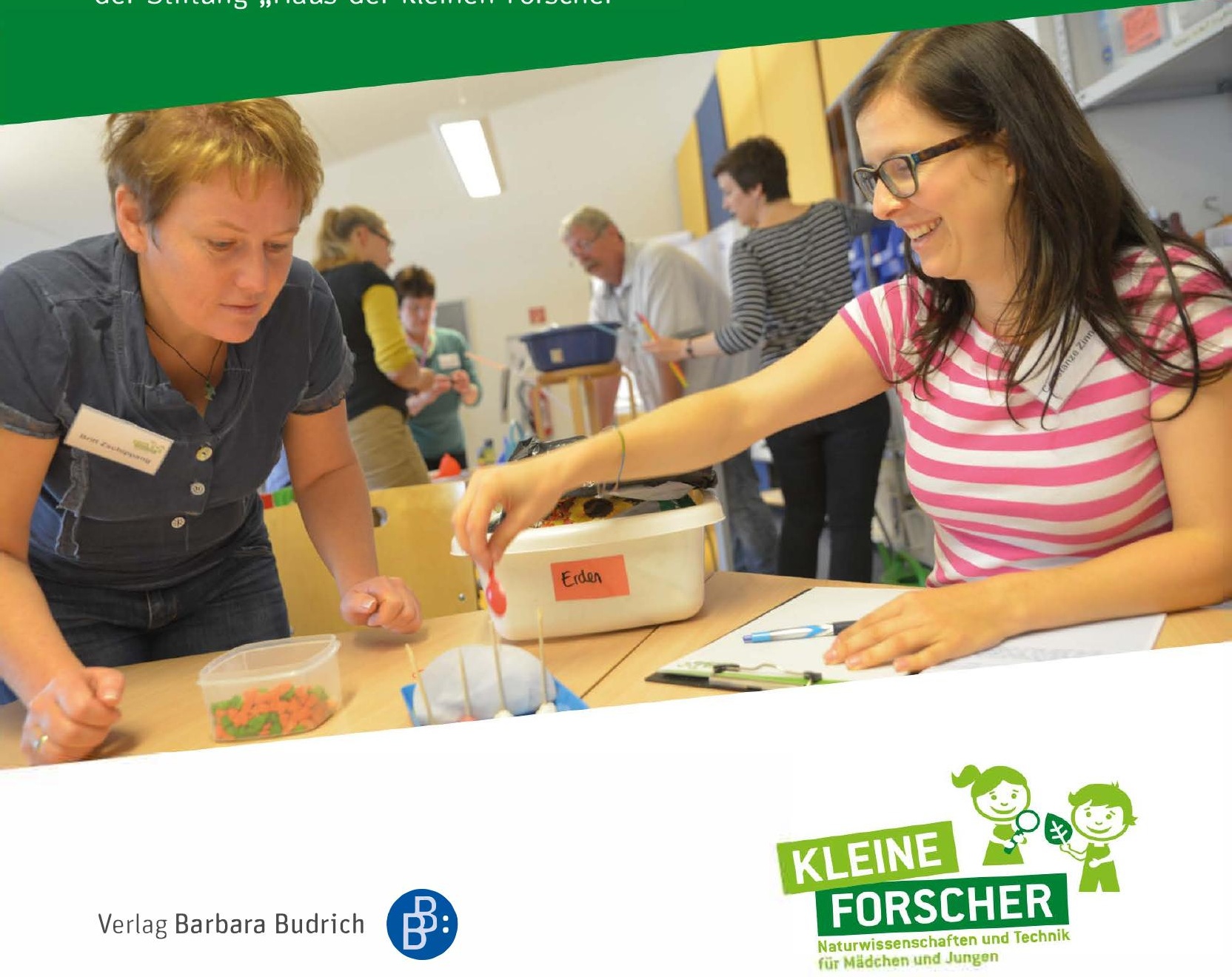


Zieldimensionen für Multiplikatorinnen und Multiplikatoren früher MINT-Bildung

Stiftung Haus der kleinen Forscher:

$$
\text { GEFÖRDERT VOM PARTNER }
$$


Wissenschaftliche Untersuchungen zur Arbeit der Stiftung „Haus der kleinen Forscher“

Band 11 
Stiftung Haus der kleinen Forscher (Hrsg.)

\section{Zieldimensionen für Multiplikatorinnen und Multiplikatoren früher MINT-Bildung}

Olaf Köller, Johannes Magenheim, Heike Molitor, Uwe Pfenning, Jörg Ramseger, Mirjam Steffensky, Christian Wiesmüller, Esther Winther, Bernd Wollring

Mit einem Geleitwort von Rudolf Tippelt

Verlag Barbara Budrich

Opladen • Berlin • Toronto 2019 
Herausgeber: Stiftung Haus der kleinen Forscher Verantwortlich: Dr. Janna Pahnke

Projektleitung: Dr. Karen Bartling, Dr. Paula Döge Konzeption und Redaktion: Dr. Christin R. Müller

Redaktionelle Mitarbeit: Ariane Ahlgrimm, Dr. Melanie Staats, Anna-Maria Tams, Dr. Sara Wagner, Claudia Wricke

Weitere Informationen finden Sie unter: www.haus-der-kleinen-forscher.de

Haben Sie Fragen, Anmerkungen oder Anregungen zu diesem Band oder der wissenschaftlichen Begleitung der Stiftungsarbeit?

Wenden Sie sich an: forschung@haus-der-kleinen-forscher.de.

Weitere Informationen und Studienergebnisse finden Sie auch unter www.haus-der-kleinen-forscher.de, Rubrik Wissenschaftliche Begleitung.

Bibliografische Information der Deutschen Nationalbibliothek

Die Deutsche Nationalbibliothek verzeichnet diese Publikation in der Deutschen Nationalbibliografie; detaillierte bibliografische Daten sind im Internet über http://dnb.d-nb.de abrufbar.

(C) 2019 Dieses Werk ist im Verlag Barbara Budrich erschienen und steht unter folgender Creative Commons Lizenz: http://creativecommons.org/licenses/ by-nc-nd/3.0/de/

Verbreitung, Speicherung und Vervielfältigung erlaubt, kommerzielle Nutzung und Veränderung nur mit Genehmigung des Verlags Barbara Budrich

Dieses Buch steht im Open-Access-Bereich der Verlagsseite zum kostenlosen Download bereit (http://dx.doi.org/10.3224/84742320).

Eine kostenpflichtige Druckversion kann über den Verlag bezogen werden. Die Seitenzahlen in der Druck- und Onlineversion sind identisch.

ISBN: $978-3-8474-2320-1$

eISBN: $978-3-8474-1352-3$

DOI: $10.3224 / 84742320$

Umschlaggestaltung: Bettina Lehfeldt, Kleinmachnow - www.lehfeldtgraphic.de Titelbildnachweis: Thomas Ernst/Stiftung „Haus der kleinen Forscher“ Lektorat und Satz: Ulrike Weingärtner, Gründau; info@textakzente.de Druck: SDK Systemdruck, Köln

Printed in Europe, gedruckt auf FSC-Papier 


\section{Inhaltsverzeichnis}

Informationen über die Autorinnen und Autoren . . . . . . . . . . 9

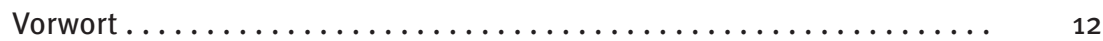

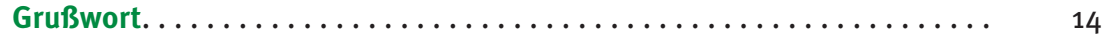

Stefan Quandt

Geleitwort..................................... 16

Rudolf Tippelt

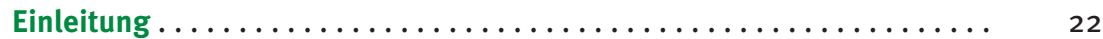

Stiftung Haus der kleinen Forscher

1 Überblick zur Stiftung „Haus der kleinen Forscher“........... 23

2 Multiplikatorinnen und Multiplikatoren in der

frühpädagogischen Weiterbildung $\ldots \ldots \ldots \ldots \ldots \ldots \ldots \ldots \ldots \ldots \ldots \ldots$

2.1 Frühe Bildung als Basis einer gelingenden Bildungsbiografie ..... 33

2.2 Fort- und Weiterbildungen im frühpädagogischen Bereich....... 34

2.3 Lehrende in der Weiterbildung frühpädagogischer Fachkräfte..... 35

3 Multiplikatorinnen und Multiplikatoren früher MINT-Bildung -

Das Projekt Trainerakademie 2.0 im „Haus der kleinen Forscher“. . . 38

3.1 Multiplikationsmodell in der Initiative

„Haus der kleinen Forscher“ ..................... 38

3.2 Das Projekt Trainerakademie 2.0 und das Qualitätssystem

für Fortbildung .......................... 40

3.3 Überblick zum vorliegenden Band................. 42

Zusammenfassung zentraler Ergebnisse ................. 44

Stiftung Haus der kleinen Forscher

Zieldimensionen für Multiplikatorinnen und Multiplikatoren

früher MINT-Bildung $\ldots \ldots \ldots \ldots \ldots \ldots \ldots \ldots \ldots \ldots \ldots \ldots \ldots \ldots \ldots$

Olaf Köller, Johannes Magenheim, Uwe Pfenning, Jörg Ramseger,

Mirjam Steffensky, Christian Wiesmüller, Esther Winther, Bernd Wollring

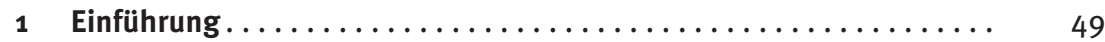

2 Theoretischer Rahmen $\ldots \ldots \ldots \ldots \ldots \ldots \ldots \ldots \ldots \ldots \ldots \ldots, 51$ 
2.1 Professionalisierung des pädagogischen Personals in Kindertagesstätten und Grundschulen .............. 51

2.2 Merkmale erfolgreicher Lehrkräftefortbildungen ............ 53

2.3 Merkmale erfolgreicher Erwachsenenbildung............ 56

3 Zieldimensionen für Multiplikatorinnen und Multiplikatoren früher MINT-Bildung $\ldots \ldots \ldots \ldots \ldots \ldots \ldots \ldots \ldots \ldots \ldots \ldots \ldots \ldots \ldots \ldots \ldots \ldots$

3.1 Motivationale Orientierungen in Bezug auf mathematische, informatische, naturwissenschaftliche und technische Bildung.... 62

3.2 Überzeugungen und Werthaltungen in Bezug auf mathematische, informatische, naturwissenschaftliche und technische Bildung.... 64

3.3 Fach- und fachdidaktisches Wissen in Mathematik, Informatik, Naturwissenschaft und Technik................... 65

3.4 Fachdidaktisches Wissen in der mathematischen, informatischen, naturwissenschaftlichen und technischen Bildung ......... 70

3.5 Allgemein-pädagogisches Wissen, Beratungswissen und Organisationswissen ....................... 74

4 Auswahl und Priorisierung der Zieldimensionen ............ 80

4.1 Fach- und fachdidaktisches Wissen in Mathematik, Informatik, Naturwissenschaft und Technik.................. 81

4.2 Allgemein-pädagogisches Wissen.................. 82

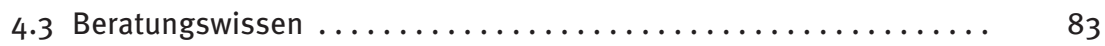

5 Fazit und Empfehlungen .......................... 84

Zieldimensionen für Multiplikatorinnen und Multiplikatoren früher MINT-Bildung aus Sicht einer Bildung für nachhaltige Entwicklung . ....................... 86 Heike Molitor

$1 \quad$ Einführung. $\ldots \ldots \ldots \ldots \ldots \ldots \ldots \ldots \ldots \ldots \ldots \ldots \ldots \ldots \ldots \ldots \ldots \ldots \ldots$

2 Zieldimensionen einer Bildung für nachhaltige Entwicklung ...... 89

3 Anforderungen an Multiplikatorinnen und Multiplikatoren einer (MINT-)Bildung für nachhaltige Entwicklung . . . . . . . 91

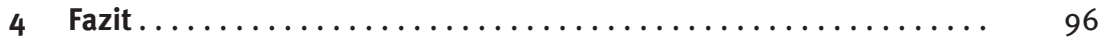


Fazit und Ausblick - Wie die Stiftung „Haus der kleinen Forscher“ mit den Erkenntnissen umgeht. . . . . . . . . . . . . . . 98

Stiftung Haus der kleinen Forscher

1 Die Zieldimensionen für Multiplikatorinnen und Multiplikatoren als Grundlage für die (Weiter-)Entwicklung der Stiftungsangebote $\ldots \ldots \ldots \ldots \ldots \ldots \ldots \ldots \ldots \ldots . \ldots . \ldots . \ldots$

2 Das Qualitätssystem für Fortbildung . ................ 103

2.1 Bewerbung, Akkreditierung und Re-Akkreditierung der

Trainerinnen und Trainer........................ 104

2.2 Qualifizierungsangebote für Trainerinnen und Trainer........... 107

2.3 Reflexionsangebote für Trainerinnen und Trainer............ 110

3 Verstetigung des Qualitätssystems und wissenschaftliche Begleitung .................. 120

$4 \quad$ Ausblick...................................... 122

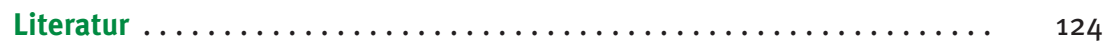

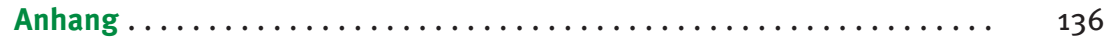

Bildquellenverzeichnis $\ldots \ldots \ldots \ldots \ldots \ldots \ldots \ldots \ldots \ldots \ldots \ldots \ldots \ldots$

Über die Stiftung „Haus der kleinen Forscher“ .............. 139

Bisher erschienen in der Wissenschaftlichen Schriftenreihe der

Stiftung, Haus der kleinen Forscher" .................. ${ }^{\circ} 141$ 



\title{
Informationen über die Autorinnen und Autoren
}

\author{
Prof. Dr. Olaf Köller
}

IPN Kiel, Abteilung Erziehungswissenschaft

Arbeitsschwerpunkte: Diagnose schulischer Kompetenzen, Individuelle Entwicklungsprozesse unter den institutionellen Rahmenbedingungen von Schule, Methodische Probleme in Large-Scale-Assessments, Bildungsmonitoring, Implementation und Evaluation von Schul- und Unterrichtsentwicklungsprogrammen Kontakt: Olshausenstraße 62, 24118 Kiel, koeller@ipn.uni-kiel.de

\section{Prof. Dr. Johannes Magenheim}

Universität Paderborn, Institut für Informatik, Fachgebiet Didaktik der Informatik Arbeitsschwerpunkte: Didaktik der Informatik, Informatik und Bildung, Kompetenzforschung, Empirische Unterrichtsforschung, E-Learning, Entwicklung und Evaluation von CSCL-Lerndesign und lernförderlicher Infrastruktur in computerbasierten Lernumgebungen

Kontakt: Fürstenallee 11, 33102 Paderborn, jsm@uni-paderborn.de

\section{Prof. Dr. Heike Molitor}

Hochschule für nachhaltige Entwicklung Eberswalde, Fachbereich Landschaftsnutzung und Naturschutz

Arbeitsschwerpunkte: Bildung für Nachhaltige Entwicklung, Umweltbildung, Globales Lernen, Naturerfahrungsräume, Nachhaltige Entwicklung, Gesundheitsförderung Kontakt: Schicklerstraße 5, 16225 Eberswalde, Heike.Molitor@hnee.de

\section{Hon.-Prof. Dr. Uwe Pfenning}

Universität Stuttgart, Deutsches Zentrum für Luft- und Raumfahrt Arbeitsschwerpunkte: Soziale Netzwerke, MINT- und Technikbildung, Sozio-MINT (sozialer, individueller und gesellschaftlicher Sinn von MINT-Bildung), EE-MINT (Bildung zur Energiewende), Bürgerbeteiligung und Partizipation, Evaluationsdesigns für MINT-Projekte

Kontakt: Brachstraße 3,73765 Neuhausen, uwe.pfenning@sowie.uni-stuttgart.de

\section{Prof. i. R. Dr. Jörg Ramseger}

Freie Universität Berlin, Fachbereich Erziehungswissenschaft und Psychologie, Arbeitsstelle Bildungsforschung Primarstufe

Arbeitsschwerpunkte: Lehr- und Lernforschung, Elementarerziehung und Primarstufenpädagogik, Schulentwicklung im Grundbildungsbereich Kontakt: Habelschwerdter Allee 45, 14195 Berlin, j.ramseger@fu-berlin.de 


\section{Prof. Dr. Mirjam Steffensky}

IPN Kiel, Abteilung Didaktik der Chemie

Arbeitsschwerpunkte: Naturwissenschaftliches Lehren und Lernen im Elementarund Primarbereich, Entwicklung und Evaluation von Materialien für den Elementar- und Primarbereich, Fachbezogenes Professionswissen von Fachkräften und Grundschullehrpersonen

Kontakt: Olshausenstraße 62, 24118 Kiel, steffensky@ipn.uni-kiel.de

\section{Prof. i. R. Dr. Rudolf Tippelt}

Ludwig-Maximilians-Universität München, Institut für Pädagogik, Bildungs- und Sozialisationsforschung, Lehrstuhl für Allgemeine Pädagogik und Bildungsforschung

Arbeitsschwerpunkte: Bildungsforschung, Weiterbildung/Erwachsenenbildung, Bildungsprozesse über die Lebensspanne, Übergang von Bildung in Beschäftigung, Fortbildung des pädagogischen Personals

Kontakt: Leopoldstraße 13, 80802 München, tippelt@edu.Imu.de

\section{Prof. Dr. Christian Wiesmüller}

Pädagogische Hochschule Karlsruhe, Institut für Physik und Technische Bildung, Technische Bildung

Arbeitsschwerpunkte: Bildungsphilosophische und schultheoretische Begründung des Faches Technik, Profilierung der Technikdidaktik als fachspezifische erziehungswissenschaftliche Disziplin, Systematisierung der Technikdidaktik als Wissenschaftsdisziplin mit Eigenprägung, Akzentuierung der emotionalen und ästhetischen Dimension des Technikunterrichts, Außerschulische Bildung, Technizität als umfassendes kulturelles Phänomen

Kontakt: Bismarckstr. 10,76133 Karlsruhe, christian.wiesmueller@ph-karlsruhe.de

\section{Prof. Dr. Esther Winther}

Universität Duisburg-Essen, Fakultät für Bildungswissenschaften, Institut für Berufs- und Weiterbildung, Fachgebiet Berufliche Aus- und Weiterbildung

Arbeitsschwerpunkte: Kompetenzen und Kompetenzentwicklung in der beruflichen Aus- und Weiterbildung, Förderung von Entrepreneurship-Kompetenzen, Soziale und gesellschaftliche Teilhabe, Professionalisierung in der Erwachsenenbildung und Weiterbildung

Kontakt: Universitätsstraße 2, 45141 Essen, esther.winther@uni-due.de 


\section{Prof. Dr. Bernd Wollring}

Universität Kassel, Fachbereich Mathematik und Naturwissenschaften, Institut für Mathematik, Didaktik der Mathematik mit Schwerpunkt Grundschule Arbeitsschwerpunkte: Lernumgebungen zur Mathematik für den Primarbereich, Lehrerausbildung und Lehrerfortbildung zu Raum und Form für den Primarbereich Kontakt: Heinrich-Plett-Straße 40, 34132 Kassel, wollring@mathematik.uni-kassel.de 


\section{Vorwort}

\section{Liebe Leserinnen und Leser,}

die Trainerinnen und Trainer in der Initiative „Haus der kleinen Forscher" setzen sich leidenschaftlich für gute frühe Bildung und forschendes Lernen ein. Das merke

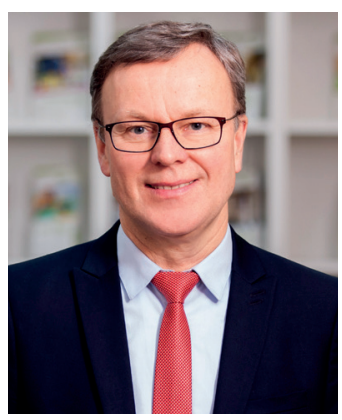
ich immer wieder in persönlichen Gesprächen. Und diese Begeisterung geben sie in den Fortbildungen weiter. Das ist ein wichtiger Faktor für den Erfolg unserer Initiative - für den Erfolg des „Hauses der kleinen Forscher".

Unsere Trainerinnen und Trainer bringen vielfältige Hintergründe und Erfahrungen für ihre Tätigkeit mit: Sie sind qualifiziert im Bildungsbereich Mathematik, Informatik, Naturwissenschaften und Technik (MINT) oder auch im Bereich einer Bildung für nachhaltige Entwicklung (BNE). Sie haben einen fundierten pädagogischen Hintergrund bzw. sind erfahrene Erwachsenenbildnerinnen und Erwachsenenbildner - manche bringen auch Erfahrungen auf allen Gebieten mit bzw. erwerben diese nach einiger Zeit beim „Haus der kleinen Forscher“. Denn wir entwickeln uns immer weiter - gemeinsam.

Wir als Stiftung „Haus der kleinen Forscher“ fragen uns immer wieder, wie wir unsere Trainerinnen und Trainer noch besser unterstützen können und was sie brauchen, um ihre Arbeit als Fortbildnerinnen und Fortbildner bestmöglich ausüben zu können, je nachdem, welche Voraussetzungen sie bereits mitbringen. Daher war es uns wichtig zu schauen, was einen guten Trainer und eine gute Trainerin ausmacht. Das Ergebnis liegt nun in diesem Band der wissenschaftlichen Schriftenreihe vor. Mein besonderer Dank gilt den Autorinnen und Autoren der Expertise, die basierend auf wissenschaftlichen Erkenntnissen aus der Lern- und Bildungsforschung, der Erwachsenenbildung und den MINT-Fachdidaktiken formuliert haben, was Multiplikatorinnen und Multiplikatoren in der frühen MINT-Bildung erfolgreich macht.

Die Definition der Zieldimensionen ist ein wichtiger Schritt für die weitere Professionalisierung unserer Trainerinnen und Trainer - und damit der Stiftung sowie der gesamten Bildungsinitiative. Denn die in diesem Band beschriebenen Zieldimensionen sind nicht nur eine Orientierung für die Trainerinnen und Trainer, in welchen Bereichen sie sich weiterentwickeln können, sondern auch für die Stiftung, um ihr Angebot für die Multiplikatorinnen und Multiplikatoren der Initiative optimal auszubauen. Dies geschieht im Rahmen eines Qualitätssystems für Fortbildung, das Trainerinnen und Trainer individuell und bedarfsgerecht bei ihrer Tä- 
tigkeit unterstützt. Eine ganz wichtige Zieldimension ist die Selbstreflexion: Dafür gibt es neue Formen der Unterstützung wie den „Kleine Forscher“-Kompass als ein digital gestütztes Werkzeug zur Reflexion der eigenen Tätigkeit oder auch Videocoaching und Hospitationen.

Das „Haus der kleinen Forscher“ versteht sich als lernende Initiative, und dieses Lernen lebt vom Engagement aller Beteiligten. Unsere Trainerinnen und Trainer wollen sich zielgerichtet weiterentwickeln. Es ist großartig, dass wir sie dabei passgenau und individuell unterstützen können. Ich freue mich sehr darauf, so gut aufgestellt, gemeinsam weiter für gute frühe Bildung für alle Kinder zu arbeiten.

Michael Fritz

Vorstandsvorsitzender der Stiftung „Haus der kleinen Forscher“ 


\section{Grußwort}

von Stefan Quandt

Der jüngste Band der Schriftenreihe der Stiftung „Haus der kleinen Forscher“ widmet sich der Arbeit der Trainerinnen und Trainer. Ohne sie und die Netzwerkpartner ist die Bildungsarbeit der Initiative in den Kitas, Horten und Grundschulen nicht denkbar. Daher ist es wichtig, die besonderen Anforderungen für Trainerinnen und Trainer genauer in den Blick zu nehmen: Über welche Kompetenzen und Fähigkeiten müssen sie verfügen, um die Bildungsimpulse der Stiftung bestmöglich an die pädagogischen Fach- und Lehrkräfte weitergeben zu können? Welche Form der Begleitung und Unterstützung seitens der Stiftung „Haus der kleinen Forscher“ brauchen sie? Und wie muss das Akkreditierungssystem für die Trainerinnen und Trainer gestaltet sein, damit die Stiftung ihre hohen Qualitätsstandards möglichst flächendeckend umsetzen kann?

Im Rahmen des Projekts Trainerakademie 2.0 hat sich die Stiftung „Haus der kleinen Forscher“ mit Unterstützung der von mir gegründeten aqtivator $\mathrm{gGmbH}$ intensiv mit diesen Fragen auseinandergesetzt. Über einen Zeitraum von gut drei Jahren wurde ein neues Qualitätssystem für Fortbildung entwickelt, das die Trainerinnen und Trainer individuell und bedarfsgerecht bei ihrer Tätigkeit unterstützt. Das Fundament dieses Qualitätssystems bilden die Zieldimensionen für Trainerinnen und Trainer, die einen wichtigen Meilenstein in der strategischen Entwicklung der Stiftung „Haus der kleinen Forscher“ markieren.

Allein der Umfang der Zieldimensionen macht deutlich, wie komplex und vielschichtig die Aufgabe der Trainerinnen und Trainer ist - und unterstreicht indirekt einmal mehr den Stellwert der Fortbildungen als zentrales Erfolgskriterium für die Stiftungsarbeit als Ganzes. Um deutschlandweit in möglichst vielen pädagogischen Einrichtungen eine anspruchsvolle frühkindliche MINT-Bildung anbieten zu können und den Qualitätsimpuls in die Fläche zu tragen, kommt es in entscheidendem Maße auf die professionelle Kompetenz der Trainerinnen und Trainer an. Ich habe daher gerne dazu beigetragen, die Stiftung „Haus der kleinen Forscher“ bei der Etablierung eines Qualitätssystems für die Aus- und Fortbildung ihrer Trainerinnen und Trainer zu unterstützen und durch die Mitwirkung der aqtivator $\mathrm{gGmbH}$ einen Beitrag zur Professionalisierung und Organisationsentwicklung der Stiftung zu leisten. Dass die Erkenntnisse aus über drei Jahren Entwicklungsarbeit mit der vorliegenden Studie nun allen Akteurinnen und Akteuren im Bereich der MINT-Bildung als Handreichung und „Best Practice“ zur Verfügung gestellt werden, passt 
gut zum Transferansatz von aqtivator, die bewährte Angebote im Bildungsbereich, insbesondere mit einem Fokus auf MINT, stärken und deren Wachstum und Verbreitung unterstützen soll.

Eine besondere Förderung des naturwissenschaftlich-technischen Nachwuchses als Grundlage für die wirtschaftliche Wettbewerbsfähigkeit und den Wohlstand unserer Gesellschaft sollte möglichst früh in der Bildungsbiographie ansetzen. Doch unser Verständnis dafür, wie elementar MINT-Bildung ist und wie vielfältig ihre Verknüpfungen mit den zentralen gesellschaftlichen wie auch ökologischen Fragestellungen unserer Zeit sind, hat in den vergangenen Jahren eine grundlegende Vertiefung und Erweiterung erfahren: MINT-Bildung zielt auf das große Ganze, indem sie zukünftige Generationen dazu befähigt, Antworten auf die großen globalen Herausforderungen der Gegenwart und Zukunft zu formulieren und zugleich den Wohlstand und damit auch den Zusammenhalt unserer Gesellschaft zu sichern. Organisationen wie die Stiftung „Haus der kleinen Forscher“, die das gesellschaftliche Bildungsengagement bündeln und den Wissenstransfer in Richtung der Kitas und Schulen koordinieren, sind dabei von großer Bedeutung.

In Anbetracht dieser Herausforderungen braucht es mehr denn je den Einsatz von Menschen, die sich mit Herz und Verstand für eine hohe Qualität in der MINT-Bildung engagieren. Die vorliegende Studie bringt dies u. a. dadurch zum Ausdruck, dass die professionelle Kompetenz der Trainerinnen und Trainer als Synthese unterschiedlichster Wissensbereiche und Fähigkeiten definiert wird, die weit über das bloße Professionswissen hinausgeht. Mit anderen Worten: die Trainerinnen und Trainer werden nicht nur als Expertinnen und Experten adressiert, sondern mit all ihrem Wissen, ihren Fähigkeiten sowie Erfahrungen und Überzeugungen in den Fortbildungsprozess einbezogen und entsprechend gefördert. Ich wünsche der Stiftung „Haus der kleinen Forscher“, dass die Trainerinnen und Trainer auf dieser Grundlage in noch stärkerem Maße dazu befähigt werden, eine umfassende Botschafterfunktion für die Initiative zu übernehmen und damit einen Beitrag zur nachhaltigen Verankerung guter MINT-Bildung in den Einrichtungen leisten. Die Trainerinnen und Trainer der Initiative „Haus der kleinen Forscher“ tragen in Zusammenarbeit mit den pädagogischen Fach- und Lehrkräften, mit der Stiftung und den Netzwerkpartnern eine zentrale Verantwortung: Ihr Erfolg entscheidet wesentlich mit über die Zukunftsperspektiven unserer Gesellschaft. Ich danke daher allen Trainerinnen und Trainern für ihr wichtiges Engagement und wünsche Ihnen hierbei allzeit viel Erfolg!

\section{Stefan Quandt}

Gründer und Gesellschafter der aqtivator gGmbH 


\section{Geleitwort}

von Rudolf Tippelt

Die Erwartungen an die Frühpädagogik sind in den letzten Jahren erheblich gestiegen: „Auf den Anfang kommt es an.“ Es wird heute zunehmend anerkannt, dass durch frühpädagogisches Engagement sowohl individuelle wie gesellschaftliche Grundlagen einer humanen, kulturellen, ökonomischen und sozialen Entwicklung gelegt werden. Das kann am besten geschehen, wenn die Qualität der Arbeit und der Leistungen in den Kitas hervorragend ist. Es stellt sich die Frage, wie man die Qualität in den einzelnen inhaltlichen Bereichen frühpädagogischer Bildung fördern kann. Wie kann man das in der so wichtigen MINT-Bildung erreichen? Und was kann das „Haus der kleinen Forscher“ hierzu beitragen?

Wenn man Multiplikatorinnen und Multiplikatoren sowie in der Folge Erzieherinnen und Kindheitspädagogen fortbilden will, wird man ein allzu selbstverständliches Idiom abwandeln müssen: „Was Hänschen oder Gretchen nicht lernen, lernen Hans oder Grete eben später.“ Anthropologische Annahmen und empirische Erkenntnisse der Erwachsenenbildungs- und der Entwicklungsforschung stützen die Prämisse, dass Menschen auch im Erwachsenenalter lernfähig und lerninteressiert sind. Es ist daher klug, bei der frühen MINT-Bildung nicht alleine bei den Kindern anzusetzen, sondern zunächst die Qualifizierung und Kompetenzentwicklung von Multiplikatorinnen und Multiplikatoren zu fördern, die ihrerseits die Professionalisierung des pädagogischen Personals in Kitas und Grundschulen stärken. Letztlich geht es doch darum, Kinder als selbstständige Akteurinnen und Akteure ihrer Bildungsbiografie zu unterstützen und die Qualität der frühen Bildung zu sichern. Zwar ist es zutreffend, dass es bereits zahlreiche Initiativen und Projekte im frühpädagogischen MINT-Bereich und seit Kurzem auch einen sinnvollen Wegweiser zur frühen naturwissenschaftlichen Bildung aus der Weiterbildungsinitiative Frühpädagogischer Fachkräfte (WIFF) gibt, und dennoch ist der Forschungs- und Fortbildungsbedarf enorm. Die vorliegende Publikation zeigt nachhaltig, dass eine weitere gründliche Auseinandersetzung mit den Aneignungsprozessen junger Kinder notwendig ist, um die professionellen Kompetenzen von Multiplikatorinnen und Multiplikatoren zu bestimmen. Die Anschlussmöglichkeiten an bereits vorliegende wissenschaftliche Erkenntnisse zur professionellen Kompetenz im frühpädagogischen Bereich in den MINT-Fächern sind bislang tatsächlich überschaubar, so dass es plausibel ist, wenn die Autoreninnen und Autoren die Ergebnisse der bereichsspezifischen Lehrkräfteprofes- 
sionsforschung und der allgemeinen Erwachsenenbildungsforschung zur Klärung der Zieldimensionen für Multiplikatorinnen und Multiplikatoren heranziehen.

Aus der Erwachsenenbildungs- und Weiterbildungsforschung weiß man, dass professionelle Kompetenz von Fortbildnerinnen und Fortbildnern auf motivationalen, volitionalen und selbstregulatorischen Ressourcen beruht und dass es darum geht, breit gelagerte wissenschaftliche Erkenntnisse in konkreten Situationen anwenden zu können. Aus der Erforschung der professionellen Lehrkompetenz kann man erschließen, dass fachliches Wissen, fachdidaktische Kenntnisse, pädagogisch-psychologisches Wissen, Beratungswissen und auch Organisationswissen zu den rechtlichen, strukturellen und finanziellen Rahmenbedingungen des Lehrens die notwendige professionelle Kompetenz von Multiplikatorinnen und Multiplikatoren umfassen. Hinzu kommen noch weitere Komponenten wie Motivation, Selbstvertrauen und ein professionelles Rollen- und Selbstverständnis. Zu Recht ist auf die fachdidaktische Kompetenz ausdrücklich zu verweisen, denn die MINT-Themen und die kognitiven und motivationalen Voraussetzungen der Lernenden müssen in Einklang gebracht werden. Dabei geht es um die traditionellen Stichworte wie z. B. Passung oder didaktische Reduktion: Das komplexe Wissen ist so einfach und klar nachvollziehbar darzustellen, dass Lernende es verstehen und sie das neue Wissen in ihre lebensweltlichen Interessen integrieren können. Zum Beratungswissen gehören aus Sicht der Erwachsenenbildung auch die Fähigkeit zur Empathie sowie die Wertschätzung der Teilnehmenden, d. h. man muss sich auf die Lernenden einlassen und sich als Lernprozessbegleitung verstehen. Letztlich geht es doch darum, durch persönliche Ausstrahlung, eine offene Haltung, eigene Neugier und Freude an der Sache, aber auch durch Authentizität, Reflexionsfähigkeit, Disziplin und Kreativität, „ein pädagogisches Feuer zu entfachen und nicht einfach einen Eimer abzufüllen“.

Auf der Basis der erwähnten Differenzierungen wird in der vorliegenden Publikation der Stiftung ein interessantes Kompetenzmodell zur mathematischen, informatischen, naturwissenschaftlichen und technischen Bildung konstruiert und für die Qualifizierung von Multiplikatorinnen und Multiplikatoren konkretisiert. Vollkommen zutreffend wird betont, dass sich wirkungsvolle Fortbildungen immer auf konkrete Themen und Inhalte aus dem übergreifenden MINT-Bereich beziehen müssen. Es handelt sich bei der Expertise um einen wichtigen Anstoß für die Professionalisierung des pädagogischen Personals im frühpädagogischen Bereich, wobei sowohl Kitas als auch Grundschulen angesprochen sind. Es werden zwar alters- und institutionendifferenzierende Aussagen formuliert, aber gleichzeitig werden Kita und Grundschule nicht segmentiert, so dass ein Gesamtbild frühpädagogischer MINT-Bildung sichtbar bleibt. 
Ohne Zweifel ist es schwierig, die frühpädagogische Forschung so zu profilieren, dass eine Nutzung wissenschaftlicher Erkenntnisse und Methoden in der Praxis und in der Bildungspolitik unmittelbar zu erkennen ist. Und dennoch gelingt es hier, die frühpädagogische Professionalisierungsforschung für die Handlungsfelder in „Wissenschaft, Praxis und Politik“ herausfordernd und interessant zu formulieren. Dies gelingt unter anderem deshalb, weil die Stiftung wissenschaftlich unabhängige und in enger Verbindung mit Hochschulen und mit außeruniversitären Forschungseinrichtungen entstehende Expertisen ermöglicht. Die vorliegende Expertise entspricht in ihrer Argumentation anerkannten exzellenten Forschungsstandards der Scientific Community, orientiert in diesem Fortbildungsbereich kreativ und gleichzeitig sachlogisch und regt praxisrelevant die Fortbildung im MINT-Bereich an.

In dem empirisch noch wenig bearbeiteten Feld kann eine forschungs- und wissensbasierte Publikation angeboten werden, die einerseits die Stiftung profiliert und andererseits sehr viele Anregungen für die Forschung in diesem domänenbezogenen Fortbildungsbereich enthält. Verantwortlich hierfür ist, dass weder reine Grundlagenforschung noch direkte Anwendungsforschung betrieben wird, sondern theoriebasiert und forschungsmethodologisch kontrolliert im konkreten Arbeitsfeld der Fortbildung von Multiplikatorinnen und Multiplikatoren wichtige und handlungsrelevante Anregungen und Konzepte erstellt werden. Die Expertise geht gleichwohl davon aus, dass pädagogische Handlungssituationen nicht monotheoretisch zu erklären sind und dass sich Multiplikatorinnen und Multiplikatoren reflexiv und aktiv mit der Wirklichkeit auseinandersetzen. Die theoretische Basis zur Einordnung praktischer Probleme ist vielfältig und die Methodenbasis zur Lösung plural. Die referierten Untersuchungen aus der schulischen Lehr- und Lernforschung sowie der Erwachsenen- und Weiterbildungsforschung sind im Feld der Frühpädagogik vielleicht nicht immer replizierbar, aber für die Lehr- und Qualitätsentwicklung frühpädagogischer Einrichtungen sowie für die Professionalisierung frühpädagogischer Fach- und Lehrkräfte in der MINT-Bildung äußerst relevant.

Erst noch zu erstellende empirische (Evaluations-)Studien werden Befunde darüber liefern, ob sich das entwickelte Kompetenzmodell im frühpädagogischen Bereich bewähren kann. Man bedarf auch des Wissens darüber, wie sich das Kompetenzmodell tatsächlich in der frühpädagogischen Weiterbildung und in den Einrichtungen realisieren lässt. Der wiederkehrende Hinweis auf die notwendigen Evaluationen der von den Multiplikatorinnen und Multiplikatoren durchgeführten pädagogischen Interventionen, dem Wissen also darüber, wie sich die umgesetzten Konzepte auf das Wissen, die Kompetenzen und die Motivation bzw. die Interessen des pädagogischen Personals und der Kinder auswirken, ist daher ein besonders wichtiger. Aber man kann die These wagen, dass Fachkräfte in frühpä- 
dagogischen Einrichtungen Kinder optimal fördern und insbesondere auch dazu beitragen können, dass Entwicklungsdefizite bei Kindern aus benachteiligten Familien durch pädagogische Interventionen und Prävention aufgeholt werden.

In den letzten Jahren wurde darüber hinaus erkannt, dass Frühpädagogik auch den Übergang in die Schule erleichtert und dass ein Bildungsauftrag von Kindertagesstätten einerseits kognitiven Kompetenzen zugutekommt, dass diese Kompetenzen andererseits in eine breite ganzheitliche Persönlichkeitsbildung der Kinder eingebettet sind. Dies trifft zweifelfrei gerade für die MINT-Bildung zu. Die enge und notwendige Zusammenarbeit von Kindergarten auf der einen Seite und dem Elternhaus auf der anderen Seite sowie dann etwas später der Grundschule werden in den Blick genommen. Die insgesamt sehr stark nachgefragte Fortbildung des frühpädagogischen Personals braucht Multiplikatorinnen und Multiplikatoren, die selbst über die zu vermittelnde Expertise in der MINT-Bildung verfügen. Aber genauso wichtig wie die konzeptionellen Grundlagen der Fortbildung von Multiplikatorinnen und Multiplikatoren sind bei der späteren Implementierung in die Fortbildungspraxis Kooperationen mit den Spitzenverbänden der Freien Wohlfahrtspflege, den kommunalen Einrichtungen, den Vertretern der Berufsverbände.

Man braucht informierte und engagierte Mitarbeitende in den frühpädagogischen Einrichtungen im MINT-Bereich, aber man braucht auch eine kompetente Führung und Leitung in diesen Einrichtungen, die die Bedeutung der MINT-Bildung im frühen Kindesalter erkennen. Voraussetzung für innovatives Arbeiten in diesem Bereich und die heute notwendige organisationale Kooperation beispielsweise mit Grundschulen ist ein bestimmtes Führungs- und Leitungshandeln, das den Sinn, die Visionen und die konkreten Ziele der MINT-Bildung mitträgt. Wichtig ist dabei, die Transparenz durch Kommunikation und möglicherweise auch durch gemeinsame Zielvereinbarungen abzusichern. Den Erzieherinnen und Erziehern sowie den Kindheitspädagogen sind Autonomie und Partizipationsmöglichkeiten zu übertragen, damit sie die in der konkreten Praxis aufkommenden multiplen Anforderungen bewältigen und meistern können. Hierzu ist auch kontinuierliche Fortbildung notwendig. Grundlegend ist also ein Typus der Leitung und Führung, der ein rational-gestaltendes Handeln, also ein intellektuell-kontrolliertes und wissenschaftlich aufgeklärtes Handeln begünstigt. Hier hat die vorliegende Publikation das praktische und wissenschaftliche Know-how für die domänenbezogenen Fortbildungen im MINT-Bereich sehr gut gebündelt und strukturiert.

Weil bei der MINT-Bildung die Kooperation verschiedener Fachdisziplinen wie der Pädagogik, der Fachdidaktik, der pädagogischen Psychologie und der Entwicklungspsychologie von großem Nutzen ist, geht es darum, das Wissen unterschiedlicher Disziplinen und Berufe aufeinander zu beziehen. Erst darauf aufbauend können dann die unterschiedlichen Kompetenzen abgeleitet werden, 
die für die MINT-Bildung ausschlaggebend sind und die letztlich dem Kindeswohl maximal dienen. Die Kompetenzen und Lehrfähigkeiten des erziehenden und bildenden Personals dabei zu fokussieren ist notwendig - das zeigt die vorliegende Publikation hervorragend.

Die sich im frühkindlichen Bereich verändernden Anforderungen der Adressatinnen und Adressaten, also vor allem der Erzieherinnen, aber auch die Heterogenität dieser Zielgruppe, erfordert eine starke Teilnehmerorientierung, wie man in der Erwachsenenbildung sagt. Die Motivation der Teilnehmenden, ihre unterschiedlichen Motive, sind immer wieder Gegenstand von Reflexionen, die Auswirkungen auf die Kurssituation und die Rolle der Multiplikatorinnen und Multiplikatoren haben. Ein hohes Interesse an fachspezifischer Fortbildung einerseits und die zielgruppenorientierte Gestaltung didaktischer Handlungsfelder andererseits ergänzen sich. Aktuelle fundierte Fachkenntnisse im Sinne eines aktuellen „domänenspezifischen Wissens“ sind gerade im MINT-Bildungsbereich notwendig, um bei Zielgruppen akzeptiert zu werden und die fachliche Urteilsfähigkeit zu gewährleisten. Betrachtet man in den allgemeinen Studien der Erwachsenenbildung den Fortbildungsbedarf, so ist dies die Stimulierung der Weiterbildungsbeteiligung der jeweiligen Zielgruppen: Fortbildung von Multiplikatorinnen und Multiplikatoren ist kein Selbstzweck, sondern Fortbildung soll über die Kompetenz der Weiterbildnerinnen und Weiterbildner die Qualität der Gestaltung von Lernkontexten verbessern und damit einen Beitrag zur Weckung von Interessen leisten.

Wenn man von einer Erhöhung der Weiterbildungsbeteiligung spricht, sollte man nicht nur die Quantität, sondern auch die Qualität der besuchten Angebote beachten. Aus der Sicht der Teilnehmenden von Fortbildungen sind die Lehrpersonen und deren professionelle Kompetenz das zentrale Qualitätskriterium. Die Kompetenzen der Multiplikatorinnen und Multiplikatoren sind Teil einer Strategie zur Förderung von Professionalität und Qualität in der Bildungsarbeit. Das trifft selbstverständlich auch im sozial und gesellschaftlich so wichtigen MINT-Bereich zu. Die Publikation der Stiftung „Haus der kleinen Forscher“ verstärkt die These, dass das Engagement schon zu einem sehr frühem Zeitpunkt der Erziehung und Sozialisation optimale Wirkungen für das heute notwendige lebenslange Lernen hat - dies gilt offenbar gerade für den MINT-Bereich.

Man wird sagen können, dass die Stiftung „Haus der kleinen Forscher“ auch durch diese Publikation zeigt, dass sie zu einem „Think Tank“ im Themenfeld der Frühpädagogik herangereift ist und innovative Forschung sowie kluge Transferaktivitäten anregt.

Prof. i. R. Dr. Rudolf Tippelt

Ludwig-Maximilians-Universität München 



\section{Einleitung}

Stiftung Haus der kleinen Forscher

Phase

Thema finden Auttrag Enitschendet ouch mit ever Guppe fin an Prarckttheino. 2 Phase:

Entwertern und Vorbereiten Auftrag Plantever Vorgeter Nutet gof die planongs pince.

1 Überblick zur Stiftung „Haus der kleinen Forscher“

2 Multiplikatorinnen und Multiplikatoren in der frühpädagogischen Weiterbildung

3 Multiplikatorinnen und Multiplikatoren in der frühen MINT-Bildung Das Projekt Trainerakademie 2.0

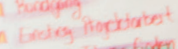

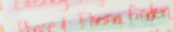

integestect

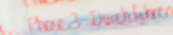

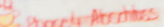
A- hetionos? A sutvint wath fhis:

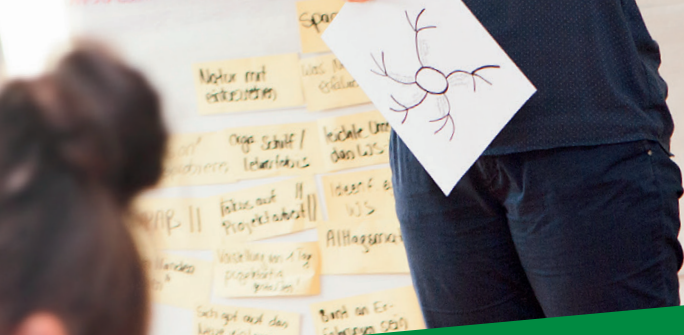

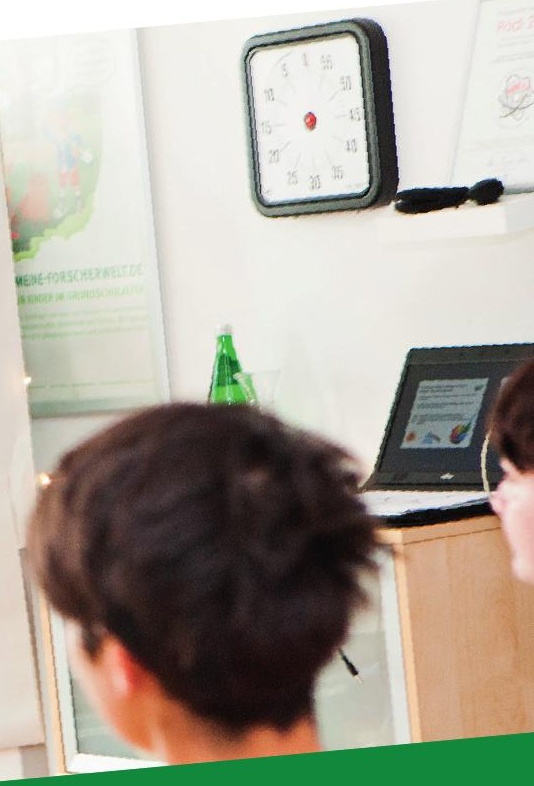




\section{1 Überblick zur Stiftung „Haus der kleinen Forscher“}

Die gemeinnützige Stiftung „Haus der kleinen Forscher“ engagiert sich seit 2006 für gute frühe Bildung in den Bereichen Mathematik, Informatik, Naturwissenschaften und Technik (MINT) - mit dem Ziel, Mädchen und Jungen stark für die Zukunft zu machen und zu nachhaltigem Handeln zu befähigen. Gemeinsam mit ihren Netzwerkpartnern vor Ort bietet die Stiftung bundesweit ein Bildungsprogramm an, das pädagogische Fach- und Lehrkräfte dabei unterstützt, Kinder im Kita- und Grundschulalter qualifiziert beim Entdecken, Forschen und Lernen zu begleiten. Das „Haus der kleinen Forscher“ verbessert Bildungschancen, fördert das Interesse an MINT und professionalisiert dafür pädagogisches Personal. Die Bildungsinitiative leistet damit einen wichtigen Beitrag in folgenden Bereichen:

- zur Qualifizierung des frühpädagogischen Personals,

- zur Qualitätsentwicklung von Einrichtungen,

- zur Persönlichkeits-, Kompetenz- und Interessenentwicklung der Kinder sowie

- zur Nachwuchsförderung in den MINT-Bildungsbereichen.

Die Hauptaktivitäten der Stiftung sind:

- der Auf- und Ausbau tragfähiger lokaler Netzwerke unter Beteiligung von Akteurinnen und Akteuren vor Ort sowie Beratung und Service für die inzwischen über 200 Netzwerkpartner,

- die Ausbildung von Multiplikatorinnen und Multiplikatoren bzw. Trainerinnen und Trainern, die vor Ort pädagogische Fach- und Lehrkräfte kontinuierlich fortbilden,

- die Entwicklung und Bereitstellung von Fortbildungskonzepten und Materialien für pädagogische Fach- und Lehrkräfte sowie

- die Unterstützung der Qualitätsentwicklung von Bildungseinrichtungen durch die Zertifizierung als „Haus der kleinen Forscher“.

\section{Qualifizierungsinitiative für Pädagoginnen und Pädagogen}

Das „Haus der kleinen Forscher“ ist bundesweit die größte Qualifizierungsinitiative für Pädagoginnen und Pädagogen im Bereich der frühen Bildung. Sie unterstützt Kitas, Horte und Grundschulen dabei, mathematische, informatische, naturwis- 
senschaftliche und/oder technische Schwerpunkte zu setzen und förderliche Entwicklungs- und Lernumgebungen für Kinder zu schaffen. Der pädagogische Ansatz der Stiftung knüpft an den Ressourcen der Kinder an und betont das gemeinsame Entdecken und Forschen im dialogischen Austausch (Stiftung Haus der kleinen Forscher, 2015a). Die Stiftung fördert mit ihren Aktivitäten auch die Umsetzung vorhandener Bildungs- und Rahmenlehrpläne der jeweiligen Bundesländer in den Bereichen Mathematik, Informatik, Naturwissenschaften und Technik.

Die inhaltlichen Angebote der Stiftung umfassen neben den Fortbildungen für pädagogische Fach- und Lehrkräfte auch pädagogische Materialien, einen jährlichen Aktionstag sowie Anregungen für Kooperationen:

- Pädagogische Materialien: Für die praktische Umsetzung in den pädagogischen Einrichtungen stellt die Stiftung in den Fortbildungen kostenlos Materialien zur Verfügung, z. B. Themenbroschüren, Forschungs- und Entdeckungskarten, didaktische Materialien und Filmbeispiele.

- Internetpräsenz: Die Website www.haus-der-kleinen-forscher.de bietet Informationen für alle Interessierten.

- Magazin „Forscht mit!“: Pädagogische Fach- und Lehrkräfte erhalten quartalsweise praktische Tipps zum Entdecken und Forschen in der Einrichtung, Informationen zur Arbeit der Stiftung sowie Best-Practice-Berichte aus anderen Einrichtungen und Netzwerken.

- „Tag der kleinen Forscher“: An diesem bundesweiten Mitmachtag können Mädchen und Jungen in ganz Deutschland ein aktuelles Forschungsthema erkunden. Dazu stellt die Stiftung den pädagogischen Einrichtungen Material bereit und ruft Unterstützerinnen und Unterstützer aus Politik, Wirtschaft, Wissenschaft und Gesellschaft zum Mitmachen auf.

- Anregungen zur Kooperation: Interessierte Eltern, Patinnen und Paten sowie andere Bildungspartner unterstützen das gemeinsame Entdecken und Forschen in den Einrichtungen.

- Zertifizierung: Engagierte Einrichtungen werden anhand festgelegter Bewertungskriterien als „Haus der kleinen Forscher“ zertifiziert. Alle sich bewerbenden Einrichtungen erhalten eine detaillierte Rückmeldung mit Anregungen für die weitere Entwicklung des gemeinsamen Entdeckens und Forschens mit den Kindern.

- Kinder-Website: Unter www.meine-forscherwelt.de gelangen Kinder im Grundschulalter in einen interaktiven Forschergarten, der sie zu eigenstän- 
digen Entdeckungsreisen animiert. Für pädagogische Fach- und Lehrkräfte stehen Tipps für die Lernbegleitung zur Verfügung.

- Service-Portal Integration: Unter integration.haus-der-kleinen-forscher.de erhalten Fach- und Lehrkräfte durch vielfältige Materialien, praxisnahe Impulse sowie inspirierenden Erfahrungsaustausch Unterstützung bei der Integration geflüchteter Kinder in Kita, Hort und Grundschule.

\section{Bundesweite Vernetzung}

Das „Haus der kleinen Forscher“ lebt als bundesweite Bildungsinitiative vom Engagement vielfältiger Akteurinnen und Akteure vor Ort - den lokalen Netzwerken, die als dauerhafte Partner und Fortbildungsanbieter in den Regionen agieren. Zu den derzeit 215 Netzwerkpartnern zählen Kommunen und Kita-Träger, Wirtschaftsverbände, Science-Center, Museen, Unternehmen, Stiftungen, Vereine usw. Seit 2011 steht das Fortbildungsprogramm der Initiative auch Horten und Ganztagsgrundschulen offen.

Circa 77.000 pädagogische Fach- und Lehrkräfte aus rund 31.400 Kitas, Horten und Grundschulen haben bereits am Fortbildungsprogramm der Initiative teilgenommen, davon pädagogische Fachkräfte aus über 25.300 Kitas sowie Fachund Lehrkräfte aus rund 1.500 Horten und rund 4.600 (Ganztags-)Grundschulen.

Deutschlandweit sind rund 5.200 Kitas, Horte und Grundschulen als „Haus der kleinen Forscher“ zertifiziert, darunter über 4.700 Kitas. Seit Herbst 2013 können sich auch Horte und Grundschulen zertifizieren lassen. Seitdem haben rund 200 Horte und über 200 Grundschulen das Zertifikat „Haus der kleinen Forscher" erhalten (Stand 31. Dezember 2018).

\section{Das kontinuierliche Fortbildungsprogramm}

Die Stiftung „Haus der kleinen Forscher“ konzentriert sich auf die Weiterqualifizierung von Pädagoginnen und Pädagogen im Hinblick auf das Entdecken und Erforschen mathematischer, informatischer, naturwissenschaftlicher und/oder technischer Themen mit Kindern. Seit 2018 gibt es auch Weiterbildungsangebote mit dem Fokus Bildung für nachhaltige Entwicklung. Das Ziel ist eine kontinuierliche Begleitung der pädagogischen Fach- und Lehrkräfte: Die Teilnahme an Fortbildungen zu unterschiedlichen Themen erweitert sukzessive das methodische Repertoire und vertieft das Verständnis des pädagogischen Ansatzes der Stiftung. Im Wechsel von Präsenzfortbildungen und Transferphasen können die Pädagoginnen und Pädagogen das Gelernte in der Praxis ausprobieren und sich dazu in der nächsten Fortbildung austauschen.

Um möglichst vielen interessierten pädagogischen Fach- und Lehrkräften die Teilnahme an Fortbildungen zu ermöglichen, findet die Weiterqualifizierung über 
ein Multiplikationsmodell statt: Die Stiftung „Haus der kleinen Forscher“ bildet an mehreren Standorten in Deutschland Trainerinnen und Trainer aus, die ihrerseits Fortbildungen für Pädagoginnen und Pädagogen in ihrem lokalen Netzwerk durchführen. Die Trainerinnen und Trainer qualifizieren sich durch die Teilnahme an den Präsenz- und Online-Fortbildungen der Stiftung dafür, Fortbildungen mit Pädagoginnen und Pädagogen durchzuführen. Als Unterstützung erhalten sie ausführliche Arbeitsunterlagen für ihre Aufgabe in der Erwachsenenbildung sowie die Möglichkeit, persönliches Feedback im Hospitationsprogramm der Stiftung oder in Form von Videocoaching zu bekommen. Für die Auffrischung und Vertiefung der Fortbildungsinhalte steht auch der Online-Campus für Trainerinnen und Trainer zur Verfügung. Die digitale Lernplattform bietet neben einer Vielzahl von Online-Lernangeboten auch inhaltliche Informationen und Arbeitsunterlagen zu den einzelnen Fortbildungsmodulen. Zu bestimmten Themen gibt es die Möglichkeit, eigenständig offene E-Learning-Module zu bearbeiten, an tutoriell begleiteten Kursen teilzunehmen sowie die Online-Begleitkurse zu Präsenzfortbildungen zu nutzen. Darüber hinaus können die Trainerinnen und Trainer in Themenforen oder offenen Chats miteinander in Kontakt treten und sich austauschen.

Sowohl für die pädagogischen Fach- und Lehrkräfte als auch für die Trainerinnen und Trainer werden in der Bildungsinitiative jedes Jahr unterschiedliche Fortbildungsthemen angeboten. Bis Ende 2016 besuchten neue Trainerinnen und Trainer bzw. erstmals teilnehmende Pädagoginnen und Pädagogen zunächst die Fortbildungen „Forschen mit Wasser“ (Workshop 1) und „Forschen mit Luft“ (Workshop 2), in denen der pädagogische Ansatz der Stiftung für das gemeinsame Entdecken und Forschen mit Kindern ausführlich thematisiert wird. Seit 2017 ist der Einstieg in das Bildungsangebot der Stiftung flexibel gestaltet. ${ }^{1}$ Sieht die Lernbegleitung noch Entwicklungsbedarf in ihrer pädagogischen Kompetenz bzw. möchte sich einen Überblick zum pädagogischen Konzept der Stiftung verschaffen, so erhält sie das Angebot, wie bisher mit den genannten Präsenzfortbildungen einzusteigen bzw. das Seminar oder den Online-Kurs „Grundlagenseminar Der pädagogische Ansatz der Stiftung ,Haus der kleinen Forscher““ zu besuchen. Ebenso können die pädagogischen Fach- und Lehrkräfte bzw. die Trainerinnen

1 Mit der Flexibilisierung des Einstiegs in das Bildungsprogramm der Stiftung nimmt die Stiftung ihre Zielgruppen in eine größere Eigenverantwortung. Gemäß dem Menschenbild eigenaktiv Lernender, das dem pädagogischen Konzept der Stiftung zugrunde liegt, setzt sie darauf, dass die pädagogischen Fach- und Lehrkräfte sowie die Trainerinnen und Trainer selbst erkennen können, wo sie mit ihren Interessen und Bedarfen stehen und welches Thema oder Format der für sie passende Einstieg ins Bil-dungsprogramm des „Hauses der kleinen Forscher“ ist bzw. welches Angebot sie für ihre weitere Professionalitätsentwicklung nutzen wollen. Um den Nutzerinnen und Nutzern der Bildungsangebote eine gute Orientierung zur bedarfsgerechten Weiterqualifizierung anzubieten, entwickelt die Stiftung digitale Reflexions- und Orientierungstools für Trainerinnen und Trainer sowie für Pädagoginnen und Pädagogen. 
und Trainer als Einstieg ein anderes Modul zu mathematischen, informatischen, naturwissenschaftlichen oder technischen Themen wählen. Die Inhalte werden in verschiedenen Formaten angeboten: Fortbildungen vor Ort, Selbstbildungsformate (wie Online-Kurse oder gedruckte pädagogische Materialien) und Bildungsveranstaltungen. Das Zertifikat „Haus der kleinen Forscher“ unterstützt darüber hinaus bei der Qualitätsentwicklung der pädagogischen Arbeit in den Einrichtungen und macht das Engagement für gute frühe MINT-Bildung nach außen sichtbar. Die Stiftung orientiert sich dabei stark an den Bedarfen, dem Vorwissen, den Vorerfahrungen und Interessen ihrer Zielgruppen.

Inhaltlich wurde das Stiftungsangebot zum Schuljahr 2017/18 mit der Fortbildung und den pädagogischen Materialien zu „Informatik entdecken - mit und ohne Computer" um den Bildungsbereich der informatischen Bildung ergänzt (zur fachlichen Fundierung siehe Band 9 der wissenschaftlichen Schriftenreihe, Stiftung Haus der kleinen Forscher, 2018b). Seit Herbst 2018 wird die Fortbildung „Technik - von hier nach da“ aus dem Bildungsbereich der technischen Bildung angeboten (Band 7, Stiftung Haus der kleinen Forscher, 2015b). Ab 2019 wird das fachübergreifende Thema „MINT ist überall“ angeboten.

Zudem wurde das Stiftungsangebot um die neuen Fortbildungen „Tür auf! Mein Einstieg in Bildung für nachhaltige Entwicklung“ und „Macht mit! - Bildung für nachhaltige Entwicklung in der Praxis“ erweitert. Die neuen Fortbildungen, Inhalte und Materialien wurden fachlich fundiert (Band 12, Stiftung Haus der kleinen Forscher, in Vorbereitung) und in 2017 und 2018 jeweils in 29 Modellnetzwerken in der Praxis erprobt. Seit 2018 bzw. 2019 stehen diese Angebote auch bundesweit pädagogischen Einrichtungen im Bereich Kita, Hort und Grundschule zur Verfügung. Erstmals wenden sich diese Angebote nicht nur an pädagogische Fach- und Lehrkräfte, sondern auch an Kitaleitungen. Zusätzlich zu den Fortbildungen und Materialien wurden Online-Kurse und Webinare zu Bildung für nachhaltige Entwicklung und zu Themen der Nachhaltigkeit sowie digitale Angebote für Kinder (Apps und Lernspiele) entwickelt.

\section{Wissenschaftliche Begleitung und Qualitätsentwicklung}

Alle Aktivitäten der Bildungsinitiative werden kontinuierlich wissenschaftlich begleitet und evaluiert. Die Stiftung „Haus der kleinen Forscher“ pflegt einen offenen Austausch mit Wissenschaft und Fachpraxis und versteht sich als lernende Organisation.

Ein umfangreiches Spektrum an Maßnahmen dient der Sicherung und Weiterentwicklung der Qualität im „Haus der kleinen Forscher“ (siehe Abbildung 1). Das stiftungseigene Qualitätsmanagement erfasst mit internen Evaluationsmaßnahmen und umfassendem Monitoring alle wichtigen Aktivitäten und Angebote. Dafür nutzt die Stiftung eine ganze Reihe an Datenquellen (wie z. B. anlassbezogene 
Befragungen der Netzwerkkoordinatorinnen und koordinatoren, der Trainerinnen und Trainer sowie der pädagogischen Fach und Lehrkräfte); eine Kombination aus quer- und längsschnittlichen Daten ermöglicht den Blick auf die aktuelle Situation und auch auf wichtige Veränderungen in den letzten Jahren. Um auf die Erkenntnisbedarfe der Stiftung flexibler reagieren zu können, wird die bisher jährliche Befragung sämtlicher Zielgruppen durch mehrere Erhebungen zu unterschiedlichen Zeitpunkten ersetzt. Die längsschnittliche Perspektive spielt in den internen Evaluations- und Monitoringmaßnahmen der Stiftung eine zunehmend wichtigere Rolle, um dem Anspruch einer stärkeren Wirkungsorientierung gerecht zu werden. Mit dem regelmäßig erscheinenden Monitoring-Bericht stellt die Stiftung wichtige Ergebnisse dieser Maßnahmen bereit. So beschreibt der Monitoring-Bericht 2016/2017 auf Grundlage einer Wirkungskette, wie das Fortbildungsangebot der Initiative zur Verbesserung der frühen MINT-Bildung in Deutschland beiträgt (Stiftung Haus der kleinen Forscher, 2017a).

Die inhaltliche (Weiter-)Entwicklung neuer Stiftungsangebote erfolgt stets kollaborativ; neue Stiftungsangebote werden also gemeinsam und im Austausch mit der Praxis entwickelt und getestet. In Zusammenarbeit mit einer Gruppe pädagogischer Fach- und Lehrkräfte aus Kitas sowie aus Horten und Grundschulen findet für jedes neue Fortbildungsangebot eine ausführliche Pilotierung statt, bevor die Fortbildungskonzepte und Materialien in den regionalen Netzwerken verbreitet werden. Dabei prüfen die mitwirkenden pädagogischen Fach- und Lehrkräfte erste Praxisideen auf ihre Umsetzbarkeit und geben Feedback zu den Unterstützungsangeboten der Stiftung. Die Fortbildungskonzepte werden auf Basis dieser Rückmeldungen überarbeitet und weiterentwickelt.

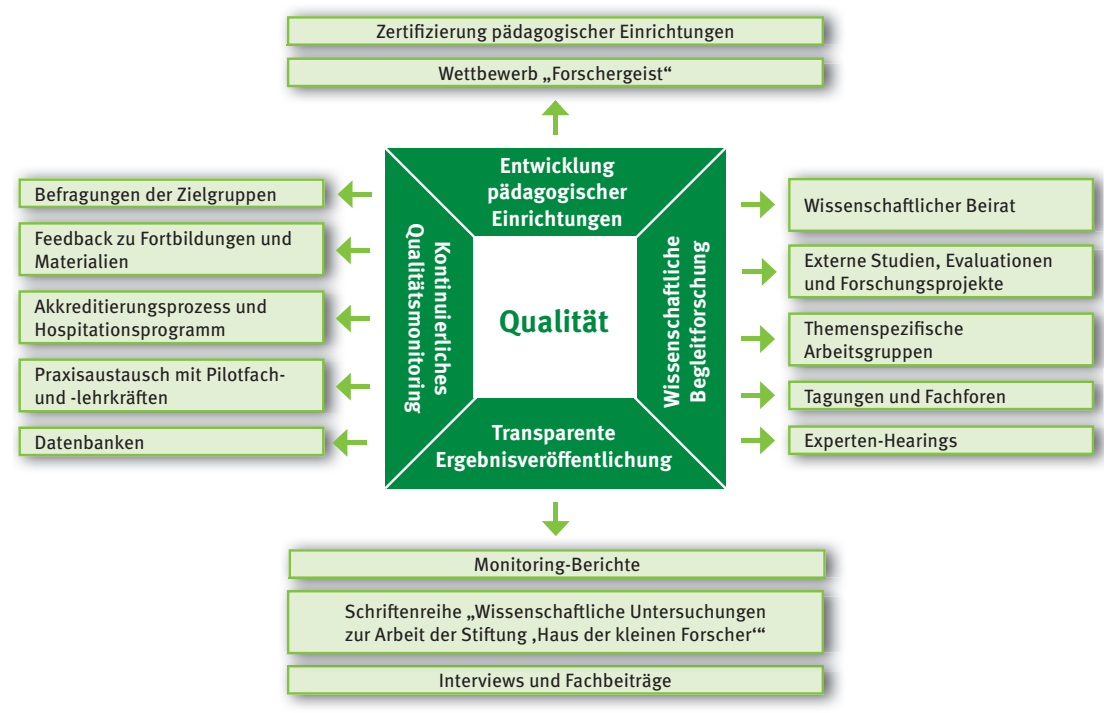

Abbildung 1. Übersicht der Maßnahmen zu Sicherung und Weiterentwicklung der Qualität der Stiftungsarbeit 
Auf Einrichtungsebene ist die Zertifizierung als „Haus der kleinen Forscher“ ein weiteres wichtiges Instrument der Qualitätsentwicklung (Stiftung Haus der kleinen Forscher, 2017b). Über die Vergabe des Zertifikats entscheidet die Stiftung in einem standardisierten Verfahren, das in Anlehnung an das Deutsche Kindergarten Gütesiegel und unter Beteiligung eines Teams aus Wissenschaftlerinnen und Wissenschaftlern (Yvonne Anders, Christa Preissing, Ursula Rabe-Kleberg, Jörg Ramseger und Wolfgang Tietze) entwickelt wurde. Die Reliabilität und Validität des Zertifizierungsverfahrens für Kitas wurde in einer externen wissenschaftlichen Studie bestätigt (Anders \& Ballaschk, 2014). Die Zertifizierung als „Haus der kleinen Forscher“ ist ein kostenfreies Verfahren zur Erfassung und Steigerung der pädagogischen Qualität bei der Umsetzung von MINT-Bildungsinhalten. Durch die Beantwortung der Fragen im Zertifizierungsfragebogen und die darauffolgende ausführliche Rückmeldung der Stiftung mit praktischen Anregungen und Tipps, werden Kitas, Horte und Grundschulen in ihrer Qualitätsentwicklung unterstützt. Eine Folgezertifizierung kann alle zwei Jahre erfolgen und ermöglicht die langfristige Verankerung und Weiterentwicklung der Bildungsqualität auf Einrichtungsebene.

Mit dem bundesweiten Kita-Wettbewerb „Forschergeist“ möchten die Deutsche Telekom Stiftung und die Stiftung „Haus der kleinen Forscher“ das Engagement der Kita-Fachkräfte sowie die Qualität der Einrichtungen würdigen. Gesucht und prämiert werden herausragende Projekte, die Mädchen und Jungen für die Welt der Mathematik, Informatik, Naturwissenschaften oder Technik begeistert haben. Mit der Ausschreibung 2018 fand der Wettbewerb bereits zum vierten Mal statt. Die prämierten Projekte werden dokumentiert und veröffentlicht, damit sie als gute Beispiele auch andere Fachkräfte für das Forschen und Entdecken in der Kita begeistern (siehe zum Beispiel Stiftung Haus der kleinen Forscher, 2016).

Neben einem kontinuierlichen Monitoring zu Zwecken der Qualitätssicherung und der Qualitätsentwicklung wird die Stiftungsarbeit im Rahmen einer langfristig angelegten externen Begleitforschung mit renommierten Partnern fachlich fundiert und in Forschungsprojekten evaluiert. Zwei unabhängige Forschungsgruppen untersuchten von 2013-2017 die naturwissenschaftlichen Bildungswirkungen in der frühen Kindheit (Stiftung Haus der kleinen Forscher, 2018a). Ziel des ersten Forschungsprojektes Early Steps into Science (kurz: EASI Science, gefördert von der Stiftung „Haus der kleinen Forscher“ und dem Bundesministerium für Bildung und Forschung) war es, Erkenntnisse über Wirkungen früher naturwissenschaftlicher Bildungsangebote auf naturwissenschaftliche Kompetenzen von pädagogischen Fachkräften und Kindern in Kitas zu gewinnen. Die Ergebnisse zeigen, dass pädagogische Fachkräfte mit naturwissenschaftlichen Fortbildungen über ein höheres Fachwissen und mehr fachdidaktische Kenntnisse verfügen als eine Vergleichsgruppe ohne Fortbildungen. Zudem sind die Motivation zu und das 
Interesse an naturwissenschaftlicher Bildung fortgebildeter Erzieherinnen und Erzieher größer. Auch die Kinder zeigen mehr Lernfreude, Interesse an Naturwissenschaften sowie Selbstvertrauen in ihr eigenes Können, wenn ihre Kita einen naturwissenschaftlichen Schwerpunkt hat. Das zweite Forschungsprojekt Early Steps into Science and Literacy (kurz: EASI Science-L, gefördert von der Stiftung „Haus der kleinen Forscher“, der Baden-Württemberg Stiftung und der Siemens Stiftung) untersuchte sprachliche Bildungswirkungen und die Interaktionsqualität im Kontext naturwissenschaftlicher Bildungsangebote. In der Studie konnte gezeigt werden, dass sich forschendes Lernen gut für die Sprachbildung eignet. Fachkräfte mit naturwissenschaftlichen Fortbildungen gestalten sprachlich anregendere Lerngelegenheiten für Kinder als Fachkräfte ohne Fortbildungen in diesem Bereich. Die sprachlichen Fähigkeiten der Kinder sind größer, wenn die sie betreuenden Fachkräfte zuvor eine kombinierte Fortbildung zu Naturwissenschaften und Sprache der Stiftung „Haus der kleinen Forscher“ besucht haben. Zudem zeigen sich positive Zusammenhänge zwischen der durch die Fachkraft gestalteten naturwissenschaftsbezogenen Prozessqualität und den naturwissenschaftlichen Kompetenzen der Kinder. Die Stiftung nutzt die Ergebnisse dieser Studien für eine systematische Reflexion ihrer bestehenden Bildungsangebote und die wirkungsorientierte Entwicklung zukünftiger Fortbildungen.

Im Hinblick auf die bedarfsorientierte Weiterentwicklung der Stiftungsangebote fördert die Stiftung derzeit gemeinsam mit dem Bundesministerium für Bildung und Forschung eine Studie zu den Entwicklungsverläufen von pädagogischen Fach- und Lehrkräften in der MINT-Bildung (EpFL MINT, Laufzeit 20172018). Ziel dieser Studie ist es, Einblicke in typische Entwicklungsverläufe in der „MINT-biografischen“ Professionalisierung pädagogischer Fach- und Lehrkräfte zu erhalten. Insbesondere sollen deren Lernbedarfe in verschiedenen Phasen ihrer Entwicklung als pädagogische MINT-Fachkraft und die Umsetzung von Lerninhalten aus Fortbildungsaktivitäten in den pädagogischen Alltag der Einrichtung untersucht werden. Die Ergebnisse der Studie sollen die Stiftung „Haus der kleinen Forscher“ dabei unterstützen, ihre Angebote zukünftig noch passgenauer und bedarfsgerechter weiterzuentwickeln.

Die Ergebnisse der wissenschaftlichen Begleitung veröffentlicht die Stiftung transparent in der vorliegenden wissenschaftlichen Schriftenreihe, alle Publikationen sind zudem über die Website frei verfügbar. ${ }^{2}$

\footnotetext{
2 Alle Ergebnisse und Publikationen zur wissenschaftichen Begleitung sind als PDF verfügbar unter: www.haus-der-kleinen-forscher.de, Rubrik „Wissenschaftliche Begleitung“. Alle Ergebnisse der externen Begleitforschung werden zudem in der vorliegenden wissenschaftlichen Schriftenreihe veröffentlicht. Eine Übersicht der bisher erschienenen Bände befindet sich auf www.haus-der-kleinen-forscher. de bzw. am Ende dieses Bandes.
} 
Ein Wissenschaftlicher Beirat berät die Stiftung zu Forschungsfragen sowie zur fachlichen Fundierung des Stiftungsangebots. Er setzt sich aus unabhängigen Wissenschaftlerinnen und Wissenschaftlern unterschiedlicher Fachgebiete zusammen und spricht Empfehlungen an den Vorstand und den Stiftungsrat aus. Die Mitglieder des Beirats sind hochkarätige Expertinnen und Experten relevanter Fachdisziplinen:

Vorsitz: Prof. Dr. Hans-Günther Roßbach, Universität Bamberg, Lehrstuhl für Elementar- und Familienpädagogik.

- Prof. Dr. Fabienne Becker-Stoll, Staatsinstitut für Frühpädagogik (IFP), München.

- Prof. Dr. Marcus Hasselhorn, Deutsches Institut für Internationale Pädagogische Forschung (DIPF), Frankfurt am Main, Abteilung Bildung und Entwicklung / Prof. Dr. Jan Lonnemann, Universität Potsdam, Lehrstuhl für Empirische Kindheitsforschung.

- Prof. Dr. Christoph Igel, Deutsches Forschungszentrum für Künstliche Intelligenz GmbH (DFKI), Abteilung Educational Technology.

- Prof. Dr. Bernhard Kalicki, Deutsches Jugendinstitut e. V. (DJI), München, Abteilung Kinder und Kinderbetreuung, und Evangelische Hochschule Dresden, Lehrstuhl für Frühkindliche Bildung.

- Prof. Dr. Alexander Kauertz, Universität Koblenz-Landau, Lehrstuhl für Physikdidaktik und Techniklehre.

- Prof. Dr. Armin Lude, Pädagogische Hochschule Ludwigsburg, Abteilung Biologie, Schwerpunkt Bildung für nachhaltige Entwicklung.

- Prof. Dr. Johannes Magenheim, Universität Paderborn, Lehrstuhl für Didaktik der Informatik.

- Prof. Dr. Jörg Ramseger, Freie Universität Berlin, Arbeitsstelle Bildungsforschung Primarstufe.

- Prof. Pia S. Schober, Ph.D, Universität Tübingen, Lehrstuhl für Soziologie mit Schwerpunkt Mikrosoziologie, und Deutsches Institut für Wirtschaftsforschung (DIW Berlin), Abteilung Bildung und Familie / Prof. Dr. C. Katharina Spieß, Freie Universität Berlin, Lehrstuhl für Bildungs- und Familienökonomie und Deutsches Institut für Wirtschaftsforschung (DIW Berlin).

- Prof. Dr. Mirjam Steffensky, Leibniz-Institut für die Pädagogik der Naturwissenschaften und Mathematik (IPN), Kiel, Lehrstuhl für Didaktik der Chemie, Schwerpunkt Frühe naturwissenschaftliche Bildung. 
- Prof. Dr. Wolfgang Tietze, PädQUIS gGmbH, An-Institut der Alice Salomon Hochschule, Berlin / Prof. Dr. Catherine Walter-Laager, Universität Graz, Arbeitsbereich Elementarpädagogik.

- Prof. Dr. Christian Wiesmüller, Pädagogische Hochschule Karlsruhe, Abteilung für Physik und Technische Bildung und Deutsche Gesellschaft für Technische Bildung (DGTB), Ansbach.

- Prof. Dr. Bernd Wollring, Universität Kassel, Lehrstuhl für Didaktik der Mathematik. 


\section{Multiplikatorinnen und Multiplikatoren in der frühpädagogischen Weiterbildung}

\subsection{Frühe Bildung als Basis einer gelingenden Bildungsbiografie}

Frühe Bildung ist die Basis einer gelingenden Bildungsbiografie und damit grundlegend für gute Entwicklungs-, Teilhabe- und Aufstiegschancen (Bundesministerium für Bildung und Forschung [BMBF], 2017). Tietze, Roßbach und Grenner (2005) zeigen beispielsweise, dass sich Kinder kognitiv positiver entwickeln, wenn sie früher und länger eine Kita besuchen. Dies gilt insbesondere für Kinder aus sozial benachteiligten oder bildungsfernen Familien (Jungmann \& Koch, 2017). Diese können in der Kita Erfahrungen mit Zahlen und Mengen, Formen, Farben, Ernährung, Sprache etc. machen. Wesentlich für diese Erfahrungen und für die kindliche Entwicklung ist das frühpädagogische Personal und dessen Kompetenzen (Anders, 2013; Blossfeld, 2013; Fukkink \& Lont, 2007; König \& Friederich, 2015; siehe Abbildung 2).

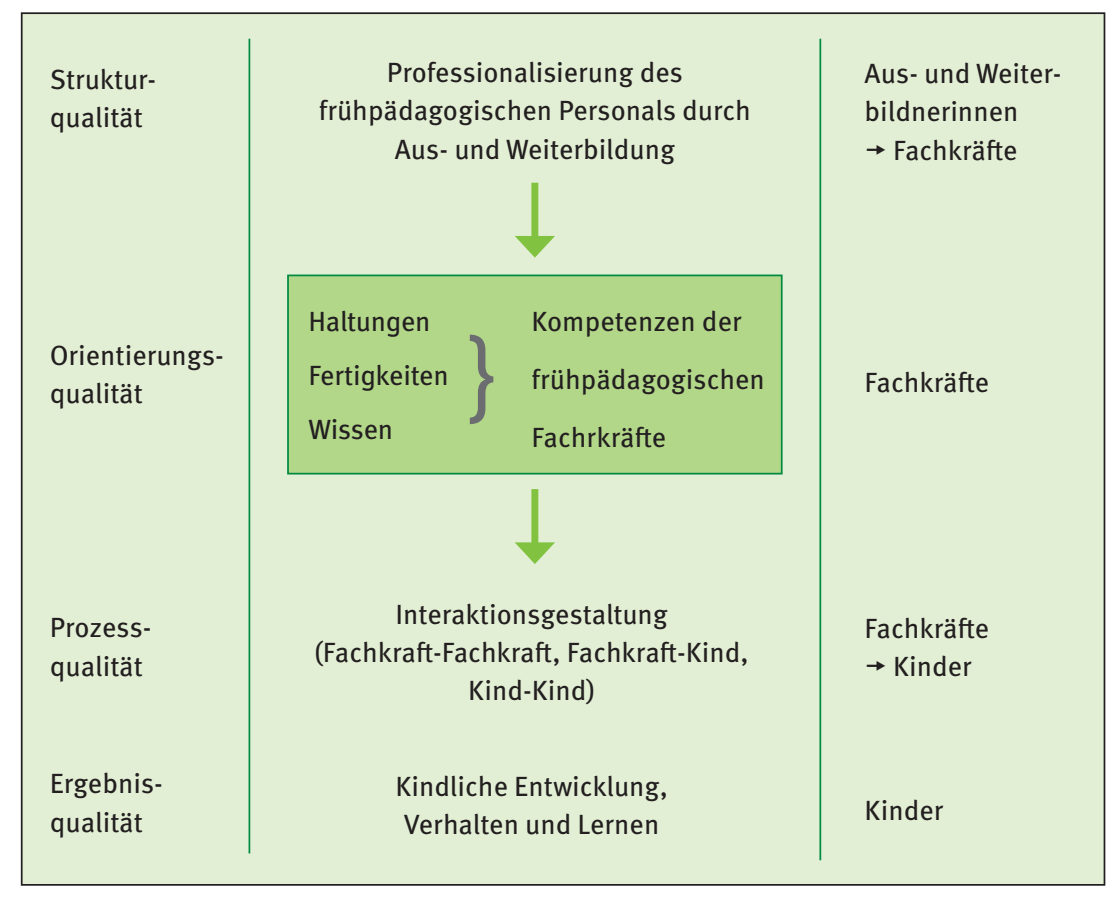

Abbildung 2. Von der Strukturqualität zur kindlichen Entwicklung, in Anlehnung an Fukkink \& Lont (2007) 
Kompetenzen sind Fähigkeiten, um komplexe Handlungsanforderungen in spezifischen Situationen zu bewältigen und eine bestimmte Qualität des Handelns zu realisieren (Fröhlich-Gildhoff, Nentwig-Gesemann, \& Pietsch, 2011). Kompetentes Handeln beinhaltet auf der einen Seite Wissen, kognitive und praktische Fähigkeiten und auf der anderen Seite Haltungen, Gefühle, Werte und Motivationen. Kompetentes Handeln wird in der Ausbildung angebahnt und später in formalen, non-formalen und informellen Lernprozessen der Fort- und Weiterbildung weiterentwickelt (Fröhlich-Gildhoff et al., 2011; Kovacevic \& Nürnberg, 2014). Fort- und Weiterbildungen gewannen insbesondere im Zuge verschiedener Reformen wie der Einführung länderspezifischer Bildungspläne und den damit veränderten Anforderungen an kompetentes und qualitätsvolles Handeln an Bedeutung (BMBF, 2017; König \& Friederich, 2015; Müller, Faas, \& Schmidt-Hertha, 2016; Starke, 2017). Beispielsweise ist die Förderung mathematischer sowie naturwissenschaftlich-technischer Basiskompetenzen fester Bestandteil der Bildungspläne aller Bundesländer. Um die Qualität dieser frühen mathematischen, naturwissenschaftlichen und technischen Förderung in Kitas zu sichern und zu steigern, braucht es Fort- und Weiterbildungen. Diese nehmen pädagogische Fachkräfte gerne an (BMBF, 2017; Stiftung Haus der kleinen Forscher, 2017a).

\subsection{Fort- und Weiterbildungen im frühpädagogischen Bereich}

Untersuchungen, u. a. der Weiterbildungsinitiative Frühpädagogische Fachkräfte (WiFF), zeigten, dass pädagogische Fachkräfte grundsätzlich eine hohe Weiterbildungsbereitschaft und beteiligung haben (Beher \& Walter, 2012; siehe auch Buschle, 2014; Rißmann, 2016; Stiftung Haus der kleinen Forscher, 2017a). Von 4.616 befragten Einrichtungsleitungen, Mitarbeiterinnen und Mitarbeitern sowie Praktikantinnen und Praktikanten haben nahezu alle in den letzten zwölf Monaten eine Fort- und Weiterbildung besucht. Dies waren vor allem kurzzeitige Veranstaltungen von bis zu drei Tagen. 15 Prozent der Befragten besuchten auch Veranstaltungen, die länger als drei Tage dauerten. Nur 2 Prozent der Befragten nutzten Fernlehrgänge oder E-Learning, jedoch 64 Prozent teambezogene Veranstaltungen wie Inhouse-Fortbildungen oder Teamtage. Trotz der hohen Weiterbildungsbeteiligung gab etwa die Hälfte der Befragten der Einrichtungsleitungen und der Mitarbeiterinnen und Mitarbeiter an, die Anzahl der Weiterbildungsangebote sollte noch erhöht werden.

Neben den verschiedenen Veranstaltungsformen gab es auch eine Vielzahl an besuchten Themen. Die häufigsten Themengebiete waren: Qualitätsentwicklung und Evaluation, Sprache, Zusammenarbeit mit Eltern, Kreativität, Zusammenarbeit mit Kindern unter drei Jahren, Mathematik, Informatik, Naturwissenschaften, 
Technik, Umwelt und Experimentieren, Beobachtung und Dokumentation, Sport, Bewegung und (Psycho)Motorik sowie Sicherheit und Erste Hilfe. Mit den behandelten Themen und Fragestellungen waren 48 Prozent der Befragten sehr zufrieden und 48 Prozent zufrieden. Mit der Kompetenz der Kursleitung waren 61 Prozent der Befragten sehr zufrieden und weitere 34 Prozent zufrieden.

Untersuchungen des „Hauses der Kleinen Forscher“ zeigen ein ähnliches Bild. Mit den jeweiligen Themen der „Haus der kleinen Forscher“-Fortbildungen sind 60 Prozent der Teilnehmenden sehr zufrieden und weitere 32 Prozent zufrieden. Mit der Atmosphäre in den Fortbildungen sind 76 Prozent sehr zufrieden und weitere 20 Prozent zufrieden. Noch zufriedener sind die Teilnehmenden mit der Kompetenz der Kursleitung. 79 Prozent sind sehr zufrieden und weitere 17 Prozent sind zufrieden (siehe auch Lau, Fritz, \& Hille, 2012 und Stiftung Haus der kleinen Forscher, 2017a).

Zusammenfassend heißt das: Die Basis einer gelingenden Bildungsbiografie sowie guter Entwicklungs-, Teilhabe und Aufstiegschancen von Kindern sind die Qualität früher Bildung und damit auch die Kompetenzen des frühpädagogischen Personals. Kompetenzen werden in der Ausbildung angebahnt und später in frühpädagogischen Fort- und Weiterbildungen weiterentwickelt. Diese nehmen pädagogische Fachkräfte regelmäßig und gerne an. Die Zufriedenheit mit den Fort- und Weiterbildungen ist sehr hoch, insbesondere die Zufriedenheit mit der wahrgenommenen Kompetenz der Kursleitung. Das ist besonders günstig, da die Kompetenz und kontinuierliche Kompetenzentwicklung der Lehrenden in der Weiterbildung maßgeblich für die Qualität der Weiterbildungsangebote ist (doppelter Praxisbezug, Buschle, 2014; Hippel \& Grimm, 2010; Iller, 2015). Die Konzentration auf die Kompetenzen der Lehrenden ist demnach zentral für die Qualitätsentwicklung von Weiterbildungsinstitutionen (Hippel \& Grimm, 2010). Dennoch ist über die Qualifikationen, Rekrutierung und Einsatzformen der Lehrenden in der Weiterbildung frühpädagogischer Fachkräfte bisher nur wenig bekannt (BMBF, 2017; Buschle, 2014; Iller, 2015).

\subsection{Lehrende in der Weiterbildung frühpädagogischer Fachkräfte}

Eine Untersuchung über das professionelle pädagogische Selbstbild von Lehrenden in der Weiterbildung frühpädagogischer Fachkräfte zeigte, dass ca. ein Drittel der Lehrenden keinen pädagogischen Abschluss haben (Buschle, 2014). 19 Prozent der Lehrenden haben ein Lehramtsstudium abgeschlossen, 21 Prozent haben eine trägerspezifische Fortbildung, 28 Prozent haben eine anderweitige pädagogische Ausbildung absolviert und 19 Prozent haben einen pädagogischen 
Studienabschluss (Mehrfachnennungen waren möglich). Untersuchungen des „Hauses der kleinen Forscher“ zeigen, dass etwa ein Viertel der Multiplikatorinnen und Multiplikatoren einen mathematischen, informatischen, naturwissenschaftlichen oder technischen beruflichen Hintergrund haben. Fast zwei Drittel haben einen pädagogisch-psychologischen beruflichen Hintergrund wie ein Studium der Sozialpädagogik oder Erziehungswissenschaft (Lau, Fritz, \& Hille, 2012; Stiftung Haus der kleinen Forscher, 2017a).

Ein pädagogisches Studium schätzen Lehrende grundsätzlich als gute Grundlage, aber nicht als hinreichend für ihre Tätigkeit ein; denn oftmals fehlen in pädagogischen Studiengängen die praktischen Erfahrungen und damit das prozedurale Wissen (Wißhak, 2017). Entsprechend führen die Weiterbildnerinnen und Weiterbildner des „Hauses der kleinen Forscher“ ihre Kompetenzen stärker auf die Teilnahme an „Haus der kleinen Forscher“-Fort- und Weiterbildungen für Multiplikatorinnen und Multiplikatoren und ihre Berufserfahrung als auf ihre Ausbildung zurück (Lau, Fritz, \& Hille, 2012). Weiterbildungsinstitutionen achten dementsprechend bei der Auswahl der Lehrenden häufig auf die praktische Erfahrung und Reputation - also Empfehlungen oder auch Rückmeldungen von Teilnehmenden - sowie auf das jeweilige Thema der Weiterbildung (BMBF, 2017; Iller, 2015; Wißhak, 2017). Demgegenüber empfiehlt Wißhak (2017), bei der Rekrutierung von Lehrenden zu überprüfen, ob professionelles Handlungswissen vorhanden ist, z. B. mit einem Wissenstest. Allerdings haben Weiterbildungsinstitutionen in der Regel keine verbindlichen Verfahren oder Kriterien zu den Anforderungen an die formale Qualifikation und zur Auswahl der Lehrenden (BMBF, 2017; Iller, 2015; Wißhak, 2017). Auch besteht meist Unklarheit über die von den Weiterbildnerinnen und Weiterbildnern bisher wahrgenommenen Weiterbildungen (Iller, 2015).

Weiterbildung ist zusätzlich zu einer Erstausbildung notwendig, um die pädagogische Professionalität voll entwickeln und den stetig verändernden Anforderungen an den frühpädagogischen Bereich gerecht werden zu können, insbesondere beim Quereinstieg aus nicht-pädagogischen Fächern (Buschle, 2014; Hippel, 2011; Wißhak, 2017). Dementsprechend sind die Lehrenden motiviert, selbst an Weiterbildungen teilzunehmen, das eigene Wissen zu festigen und zu erweitern und Ideen für neue Themen oder die methodisch-didaktische Gestaltung der eigenen Weiterbildungen zu gewinnen (Buschle, 2014). „Fortbildung der [Weiterbildnerinnen und] Weiterbildner ist damit nicht Selbstzweck, sondern dient der verbesserten Gestaltung von Lernkontexten Erwachsener und will damit den Aufgaben von Erwachsenenbildung (Förderung der individuellen Kompetenz, ökonomischen Innovation, sozialen Integration und kulturellen Partizipation) nachkommen“ (Tippelt, Hippel, \& Fuchs, 2009, 13). Deshalb haben auch die Weiterbildungsträger ein Interesse daran, dass sich die Lehrenden weiterbilden. Die Verantwortung 
dafür sehen sie aber zumeist bei den Lehrenden selbst und achten nur vereinzelt auf deren Weiterbildungsverhalten (Buschle, 2014; Iller, 2015). Die Stiftung „Haus der kleinen Forscher“ bietet den Weiterbildnerinnen und Weiterbildnern der Initiative kostenlose Fortbildungen zu mathematischen, informatischen, naturwissenschaftlichen, technischen sowie methodisch-didaktischen Themen und erfasst die Teilnahme an diesen Angeboten (Stiftung Haus der kleinen Forscher, 2017a). Wißhak (2017) empfiehlt demgemäß, dass Träger auf der regelmäßigen Weiterbildung ihrer Lehrenden bestehen und entsprechende Materialien und Weiterbildungsangebote selbst entwickeln und anbieten. So können die Träger gewährleisten, dass fundierte Modelle und aktuelle empirische Befunde einbezogen werden. Indes ist häufig unklar, was der Outcome der Materialien und Weiterbildungsangebote sein soll, welche Kompetenzen dadurch gefördert werden sollen und wie diese Kompetenzentwicklung nachvollzogen werden kann. Denn empirisch überprüfte und verbindliche Regelungen über Mindestanforderungen oder Qualitätsstandards für Lehrende bzw. Trainerinnen und Trainer fehlen bislang (Buschle, 2014; Wißhak, 2017).

Demzufolge ist es notwendig, ein Kompetenzmodell für Multiplikatorinnen und Multiplikatoren in der frühpädagogischen Weiterbildung zu formulieren. Die Aufgaben und Tätigkeiten von Lehrenden bzw. Trainerinnen und Trainern müssen analysiert und die daraus abgeleiteten Zieldimensionen präzisiert werden (Buschle, 2014). Denn Kompetenzmodelle vermitteln zwischen abstrakten konkreten Aufgabenstellungen bzw. Handlungsanforderungen und Zieldimensionen. Sie dienen dazu, abstrakte Ziele auf Basis fachlicher, fachdidaktischer sowie pädagogisch-psychologischer Konzepte zu konkretisieren und messbar zu machen (Fröhlich-Gildhoff et al., 2011). Entsprechend beinhaltet das GRETA-Kompetenzmodell für Lehrende in der Erwachsenen- und Weiterbildung berufspraktisches Wissen und Können, fach- und feldspezifisches Wissen, professionelle Selbststeuerung sowie professionelle Werthaltungen und Überzeugungen (Lencer \& Strauch, 2016; siehe auch Baumert \& Kunter, 2006). Das fach- und feldspezifische Wissen ist unterteilt in den Fachinhalt und den Feldbezug, allerdings im Modell nicht weiter ausdifferenziert und daher nicht ohne Weiteres auf die frühe MINT-Bildung übertragbar.

Die Stiftung „Haus der kleinen Forscher“ hat dieses Desiderat aufgegriffen und gemeinsam mit Expertinnen und Experten aus Wissenschaft und Praxis Kompetenzen bzw. Zieldimensionen für Multiplikatorinnen und Multiplikatoren früher MINT-Bildung konkretisiert. Darauf aufbauend hat die Stiftung im Laufe des Projekts Trainerakademie 2.0 ein Qualitätssystem für Fortbildung entwickelt. Dieses wird im Fazitkapitel des vorliegenden Bands ausführlich erläutert. 


\section{Multiplikatorinnen und Multiplikatoren früher MINT-Bildung - Das Projekt Trainerakademie 2.0 im „Haus der kleinen Forscher"}

\subsection{Multiplikationsmodell in der Initiative „Haus der kleinen Forscher“}

Die Stiftung „Haus der kleinen Forscher“ engagiert sich für gute frühe Bildung in den Bereichen Mathematik, Informatik, Naturwissenschaften, Technik und Nachhaltigkeit - mit dem Ziel, Mädchen und Jungen stark für die Zukunft zu machen und zu nachhaltigem Handeln zu befähigen. Die Stiftung bietet hierfür bundesweit Fort- und Weiterbildungen an, die pädagogische Fach- und Lehrkräfte dabei unterstützen, Kinder im Kita- und Grundschulalter qualifiziert beim Entdecken und Forschen zu begleiten (siehe Kapitel 1 Überblick zur Stiftung „Haus der kleinen Forscher"). Die Entwicklung dieser Fort- und Weiterbildungen erfolgt stets auf Basis einer fachlichen Fundierung und im Austausch mit der Zielgruppe.

Im Rahmen der fachlichen Fundierung haben in den letzten Jahren von der Stiftung initiierte interdisziplinäre Expertengruppen auf Basis des aktuellen wissenschaftlichen Forschungsstands pädagogisch-inhaltliche Zieldimensionen zu den verschiedenen Facetten früher MINT-Bildung formuliert. Bisher wurden Zieldimensionen früher naturwissenschaftlicher (Band 5), technischer (Band 7), mathematischer (Band 8) sowie informatischer (Band 9) Bildung für Kinder und pädagogische Fach- und Lehrkräfte im Elementar- und Primarbereich erarbeitet und in der wissenschaftlichen Schriftenreihe der Stiftung publiziert (Stiftung Haus der kleinen Forscher, 2013, 2015b, 2017c, 2018b). Zuletzt formulierte eine Expertinnen- und Expertengruppe Zieldimensionen einer Bildung für nachhaltige Entwicklung für Kinder und pädagogische Fach, Lehr- und Leitungskräfte (Kauertz, Molitor, Saffran, Schubert, Singer-Brodowski, Ulber, \& Verch, in Vorbereitung).

Aufbauend auf diesen Zieldimensionen werden die Stiftungsangebote entwickelt, also Fort- und Weiterbildungen sowie pädagogische Materialien - und zwar gemeinsam und im Austausch mit pädagogischen Fach- und Lehrkräften aus den Piloteinrichtungen der Stiftung. Dabei prüfen die pädagogischen Fach- und Lehrkräfte erste Praxisideen auf ihre Umsetzbarkeit und geben Feedback zu den Unterstützungsangeboten der Stiftung. Zudem finden regelmäßige Befragungen und Evaluationen statt, in der pädagogische Fach- und Lehrkräfte ihre Bedarfe und Rückmeldungen einbringen können. Die Fortbildungskonzepte und Materialien werden auf Basis dieser Rückmeldungen überarbeitet und weiterentwickelt 
und danach über das Multiplikationsmodell in den regionalen Netzwerken zur Verfügung gestellt. Pädagogische Fach- und Lehrkräfte in den regionalen Netzwerken in ganz Deutschland können so an Fortbildungen des „Hauses der kleinen Forscher“ teilnehmen, die von den Multiplikatorinnen und Multiplikatoren des „Hauses der kleinen Forscher“, den so genannten Trainerinnen und Trainern, durchgeführt werden (siehe Kapitel 1 Überblick zur Stiftung „Haus der kleinen Forscher“).

Die derzeit rund 600 Trainerinnen und Trainer (Stand 31. Dezember 2018) haben eine wichtige Multiplikationsfunktion innerhalb der Initiative „Haus der kleinen Forscher“. Finanziert von den Netzwerkpartnern, geben sie im Schnitt seit fünf Jahren Fortbildungen für pädagogische Fach- und Lehrkräfte. Darüber hinaus leisten sie an vielen Stellen auch wertvolle Unterstützungsarbeit im Netzwerk vor Ort, etwa mit Prozessbegleitungen oder fachlichen Zusatzangeboten für die pädagogischen Einrichtungen. Sie tragen damit zur Professionalisierung des Netzwerks und zur Qualitätsentwicklung der Arbeit in den Einrichtungen bei (siehe auch Stiftung Haus der kleinen Forscher, 2012, 2017a). Zudem bringen sich die Trainerinnen und Trainer in verschiedenen Austauschformaten in die (Weiter-)Entwicklung der Angebote der Initiative mit ein (Teilnahme an Veranstaltungen, Befragungen, Fokusgruppen, Online-Austausch u.a.m.). Fast zwei Drittel der Trainerinnen und Trainer haben einen pädagogisch-psychologischen beruflichen Hintergrund wie ein Studium der Sozialpädagogik oder Erziehungswissenschaft; etwa ein Viertel bringen einen mathematischen, informatischen, naturwissenschaftlichen oder technischen beruflichen Hintergrund mit (Lau, Fritz, \& Hille, 2012; Stiftung Haus der kleinen Forscher, 2017a).

Für ihre Arbeit als Weiterbildnerinnen und Weiterbildner qualifizieren sich die Trainerinnen und Trainer durch die Teilnahme an den Fortbildungen der Stiftung. Zudem erhalten sie ausführliche Arbeitsunterlagen zum Thema Erwachsenenbildung oder auch persönliches Feedback im Rahmen einer Hospitation. Die Fortbildungen und Materialien sind qualitativ hochwertige, aber auch für alle Trainerinnen und Trainer einheitliche Angebote. Das heißt, auf individuell spezifische Bedarfe der Trainerinnen und Trainer können die Angebote der Stiftung bislang nur eingeschränkt reagieren. Steffensky, Anders, Barenthien, Hardy, Leuchter, Oppermann, Taskinen und Ziegler (2018) empfehlen angesichts der individuell spezifischen Bedarfe der Trainerinnen und Trainer, die Kompetenzen der Trainerinnen und Trainer sowie die Schwerpunkte und die Qualität der von den Trainerinnen und Trainern durchgeführten Fortbildungen genauer zu untersuchen, um eine entsprechende Qualitätssicherung und Qualitätsentwicklung der Fortbildungen betreiben zu können. Diese Empfehlung greift die Stiftung „Haus der kleinen Forscher" im Rahmen des Projekts Trainerakademie 2.0 auf und widmet sich einer verbesserten und bedarfsgerechteren Qualifizierung und Unterstützung der Trainerinnen und Trainer in der Initiative. 


\subsection{Das Projekt Trainerakademie 2.0 und das Qualitätssystem für Fortbildung}

Das Projekt Trainerakademie 2.0 ist eine dreijährige Qualitätsoffensive, die vom 1. Januar 2016 bis zum 28. Februar 2019 von der aqtivator $\mathbf{g G m b H}{ }^{3}$ gefördert wurde. Ziel der Qualitätsoffensive war eine stärker am individuellen Bedarf orientierte Qualifizierung der Trainerinnen und Trainer und die Qualitätssicherung und Qualitätsentwicklung der Fortbildungsangebote der Stiftung „Haus der kleinen Forscher“ für pädagogische Fachkräfte aus Kita, Hort und Grundschule.

Hierfür wurde ein System für die Qualitätssicherung und -entwicklung der Fortbildungen geschaffen. Wesentliche Elemente des Systems betreffen die Bewerbung und Akkreditierung zu Beginn der Tätigkeit als Trainerin bzw. Trainer, bedarfsgerecht gestaltete Qualifizierungsphasen sowie eine alle zwei Jahre wiederkehrende Re-Akkreditierung. Der Ansatz einer möglichst individuellen und bedarfsgerechten Unterstützung, den die Stiftung auf Ebene der Kinder wie der pädagogischen Fach- und Lehrkräfte verfolgt, findet damit auch auf der Ebene der Trainerinnen und Trainer Anwendung (Stiftung Haus der kleinen Forscher, 2015a, 2019).

Grundlage des Systems für die Qualitätssicherung und -entwicklung der Fortbildungen ist ein Kompetenzmodell, das aufbauend auf den Zieldimensionen mathematischer, informatischer, naturwissenschaftlicher und technischer Bildung für Kinder und pädagogische Fach- und Lehrkräfte (Stiftung Haus der kleinen Forscher, 2013, 2015b, 2017c, 2018b) bewusst auf Ebene der Trainerinnen und Trainer die Zieldimensionen für Multiplikatorinnen und Multiplikatoren früher MINT-Bildung konkretisiert. Denn eine Voraussetzung für die bedarfsgerechte Auswahl, Qualifizierung und Begleitung ist eine klare Zielorientierung, was gute Trainerinnen und Trainer können sollen und welche Kompetenzen sie dafür benötigen (siehe auch Buschle, 2014 und Kapitel 2.3 Lehrende in der Weiterbildung frühpädagogischer Fachkräfte). Die Stiftung hat 2016/2017 hierzu eine Gruppe wissenschaftlicher Expertinnen und Experten der Frühpädagogik, der Bildungsforschung, der Erwachsenenbildung, der Kompetenzerfassung und der MINT-Fachdidaktiken initiiert:

3 Der Projektpartner aqtivator $g \mathrm{GmbH}$ fördert gemeinnützige Organisationen, die sich erfolgreich für Bildung, Integration und Chancengerechtigkeit einsetzen. aqtivator unterstützt die Professionalisierung und das Wachstum der geförderten Organisationen. Ein besonderer Fokus liegt dabei auf der Entwicklung nachhaltiger Skalierungsmodelle. Gründer und Gesellschafter von aqtivator ist der Unternehmer Stefan Quandt. 
- Prof. Dr. Olaf Köller (IPN, Kiel),

- Prof. Dr. Johannes Magenheim (Universität Paderborn),

- Prof. Dr. Uwe Pfenning (DZLR, Stuttgart),

- Prof. Dr. Jörg Ramseger (Freie Universität Berlin),

- Prof. Dr. Mirjam Steffensky (IPN, Kiel),

- Prof. Dr. Christian Wiesmüller (Pädagogische Hochschule Karlsruhe),

- Prof. Dr. Esther Winther (DIE, Bonn) und

- Prof. Dr. Bernd Wollring (Universität Kassel).

Die Expertinnen und Experten diskutierten in mehreren Arbeitstreffen gemeinsam mit der Stiftung, welche Kompetenzen gute Trainerinnen und Trainer in der frühen MINT-Bildung benötigen, und sichteten vorhandene Messinstrumente zur Erfassung dieser Kompetenzen. Darüber hinaus fanden Fokusgruppen mit Praxisvertreterinnen und -vertretern statt, bei denen Trainerinnen und Trainer sowie Netzwerkkoordinatorinnen und -koordinatoren ihre Sicht einbrachten, welche Kenntnisse, Fähigkeiten und Voraussetzungen Trainerinnen und Trainer benötigen, um gute Fortbildungen zur frühen MINT-Bildung durchzuführen. Die Ergebnisse der Fokusgruppen wurden in das Kompetenzmodell einbezogen. Schließlich wurden das Kompetenzmodell sowie das im Projekt Trainerakademie 2.0 entwickelte Qualitätssystem für Fortbildung mehrfach im Wissenschaftlichen Beirat der Stiftung diskutiert. Die Beiratsmitglieder begrüßten die Entwicklung des Qualitätssystems und gaben kritische Empfehlungen zu dessen Ausgestaltung, Einführung und Reflexion.

Das Kompetenzmodell diente als Basis für die Entwicklung des Systems für die Qualitätssicherung und -entwicklung der Fortbildungen. Es sollte Orientierung für die Bewerbungs-, Akkreditierungs- und Re-Akkreditierungsanforderungen sowie für die am individuellen Bedarf orientierte Qualifizierung und Unterstützung bieten. Darüber hinaus sollte es Anknüpfungspunkte für eine mögliche Wirkungsmessung der Stiftungsangebote für Trainerinnen und Trainer bieten.

Im Laufe des Projekts wurden die bestehenden Qualifizierungsangebote weiterentwickelt und neue Qualifizierungsangebote erarbeitet, z. B. Online-Angebote, Präsenzfortbildungen zur Gestaltung von Seminaren in der Erwachsenenbildung oder netzwerkinterne Fortbildungen, die vor Ort in den Netzwerken angeboten werden. Insbesondere sollten die bisher vorrangig analogen Qualifizierungsformate durch digitale Angebote ergänzt werden. Zudem sollte ein videogestütztes, zeitlich und räumlich flexibles Beratungsinstrument für Videocoaching entwickelt 
werden (Video Interactive Guidance), um gemeinsam mit den Trainerinnen und Trainern die Qualität der Interaktion mit den pädagogischen Fach- und Lehrkräften zu analysieren und zu reflektieren. Denn Veränderungen im professionellen Handeln sind nur dann wirklich nachhaltig, wenn theoretisch Erlerntes immer wieder in der Praxis angewendet, danach reflektiert und schließlich erneut angewendet wird (Sandwich-Prinzip, Wahl, 2013). Um die Trainerinnen und Trainer dabei zu unterstützen, die Qualifizierungsangebote ihrem individuellen Bedarf entsprechend auszuwählen, sollte ein internetbasiertes Reflexions- und Orientierungstool entwickelt werden. Dieses soll den Trainerinnen und Trainern eine Rückmeldung und Empfehlungen dazu geben, welche Angebote der Stiftung sie dabei unterstützen können, sich gezielt weiterzubilden.

\section{3 Überblick zum vorliegenden Band}

Der elfte Band der Schriftenreihe „Wissenschaftliche Untersuchungen zur Arbeit der Stiftung ,Haus der kleinen Forscher““ umfasst die von Olaf Köller, Johannes Magenheim, Uwe Pfenning, Jörg Ramseger, Mirjam Steffensky, Christian Wiesmüller, Esther Winther und Bernd Wollring erarbeitete Expertise „Zieldimensionen für Multiplikatorinnen und Multiplikatoren früher MINT-Bildung“. Die Expertise beschreibt und priorisiert die Kompetenzen, die gute Trainerinnen und Trainer in der frühen MINT-Bildung auszeichnen, und gibt Empfehlungen für die (Weiter-) Entwicklung der Stiftungsangebote. Heike Molitor reflektiert in einem ergänzenden Kapitel die Anforderungen an Multiplikatoren früher MINT-Bildung aus Sicht einer Bildung für nachhaltige Entwicklung (siehe auch Stiftung Haus der kleinen Forscher, in Vorbereitung). Im Fazit des Bandes beschreibt die Stiftung die Umsetzung der Zieldimensionen und der Empfehlungen in die Stiftungsarbeit und das Qualitätssystem für Fortbildung. 



\section{Zusammenfassung zentraler Ergebnisse}

Stiftung Haus der kleinen Forscher

- erwartung

- Menge an Mcoterial

- hohe Uor Ingse

- Aifoar 


\section{Zusammenfassung zentraler Ergebnisse}

Der vorliegende elfte Band der Schriftenreihe „Wissenschaftliche Untersuchungen zur Arbeit der Stiftung ,Haus der kleinen Forscher“" fokussiert die Zieldimensionen für Multiplikatorinnen und Multiplikatoren früher MINT-Bildung, auch mit Blick auf eine Bildung für nachhaltige Entwicklung. Der Band umfasst erstens Ausführungen zum Hintergrund des Projekts Trainerakademie 2.0 in der Stiftung „Haus der kleinen Forscher“, zweitens eine wissenschaftliche Expertise zu den „Zieldimensionen für Multiplikatorinnen und Multiplikatoren früher MINT-Bildung“, die von einer interdisziplinären Expertinnen- und Expertengruppe erstellt wurde sowie drittens einen ergänzenden Kommentar aus Sicht einer Bildung für nachhaltige Entwicklung. Die fachlichen Empfehlungen bilden die konzeptionelle Grundlage für die viertens beschriebene (Weiter-)Entwicklung der Angebote für Multiplikatorinnen und Multiplikatoren - den so genannten Trainerinnen und Trainern - in der Initiative „Haus der kleinen Forscher“, z. B. Bewerbungs-, Akkreditierungs- und Re-Akkreditierungsverfahren, Qualifizierungs- und Reflexionsangebote.

Die Expertise „Zieldimensionen für Multiplikatorinnen und Multiplikatoren früher MINT-Bildung“von Olaf Köller, Johannes Magenheim, Uwe Pfenning, Jörg Ramseger, Mirjam Steffensky, Christian Wiesmüller, Esther Winther und Bernd Wollring beschreibt ein Modell professioneller Kompetenz und Zieldimensionen für Multiplikatorinnen und Multiplikatoren früher MINT-Bildung. Die Zieldimensionen basieren u. a. auf den Merkmalen erfolgreicher Lehrkräfte- und Erwachsenenbildung und beschreiben, was gute Trainerinnen und Trainer in der frühen MINT-Bildung, gerade in Bezug auf ihre Rolle in der Erwachsenenbildung, auszeichnet:

- Motivation und Selbstvertrauen,

- Professionelles Rollen- und Selbstverständnis,

- Fach- und Vorgehenswissen in Mathematik, Informatik, Naturwissenschaft und Technik,

- Fachdidaktisches Wissen über pädagogische Handlungsstrategien sowie

- Pädagogisches Wissen, Beratungs- und Organisationswissen. 
Die Expertise enthält darüber hinaus Empfehlungen zur Auswahl und Priorisierung der Zieldimensionen. Als besonders bedeutend heben die Autorinnen und Autoren die Zieldimensionen Motivation und Professionswissen hervor. Die Expertise beinhaltet schließlich auch Empfehlungen für die (Weiter-)Entwicklung der Stiftungsangebote und die wissenschaftliche Begleitung der Stiftungsarbeit. Empfohlen wird, die professionellen Kompetenzen der Trainerinnen und Trainer zu erfassen, die bestehenden Qualifizierungsangebote für Trainerinnen und Trainer damit abzugleichen und passgenau weiterzuentwickeln und die Qualität der Fortbildungen sowie deren Wirkungen zu erfassen. Die Fortbildungen sollten neben einem Wissensaufbau vor allem auch die Entwicklung einer Haltung und Handlungskompetenz anbahnen, die dem pädagogischen Ansatz der Bildungsinitiative zum entdeckenden und forschenden Lernen entspricht.

Heike Molitor diskutiert in ihrem Kommentar die für Multiplikatorinnen und Multiplikatoren der frühen MINT-Bildung empfohlenen Kompetenzen aus Sicht einer Bildung für nachhaltige Entwicklung. Hierbei stellt sie eine Reihe von Überschneidungen relevanter Zieldimensionen fest und hebt Aspekte hervor, die in Bezug auf die Beförderung einer nachhaltigen Entwicklung in pädagogischen Einrichtungen wie auch in der Gesellschaft von besonderer Bedeutung sind. Das Kapitel zeigt auf, dass MINT-Bildung und Bildung für nachhaltige Entwicklung in einem konstruktiven Miteinander gegenseitige Reflexionsprozesse anregen können, und beschreibt, welchen Anforderungen Multiplikatorinnen und Multiplikatoren im Kontext einer solchen (MINT-)Bildung für nachhaltige Entwicklung begegnen.

Das Fazit des Bandes beschreibt die Umsetzung der wissenschaftlichen Empfehlungen in die Angebote der Initiative „Haus der kleinen Forscher“. Hierbei werden die Ergebnisse des Projekts Trainerakademie 2.0, wie das überarbeitete Bewerbungs-, und Akkreditierungs- und Re-Akkreditierungsverfahren für Trainerinnen und Trainer und die Weiterentwicklung der Qualifizierung, Begleitung und Unterstützung dieser Multiplikatorinnen und Multiplikatoren, beschrieben. Zudem gibt es einen Ausblick auf die weitere Evaluation und wissenschaftliche Begleitung der Stiftungsarbeit. 



\section{Zieldimensionen für Multiplikatorinnen und Multiplikatoren früher MINT-Bildung}

Olaf Köller, Johannes Magenheim, Uwe Pfenning, Jörg Ramseger, Mirjam Steffensky, Christian Wiesmüller, Esther Winther, Bernd Wollring

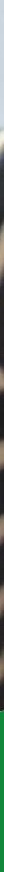

1 Einführung

2 Theoretischer Rahmen

3 Zieldimensionen für Multiplikatorinnen und Multiplikatoren früher mathematischer, informatischer, naturwissenschaftlicher und technischer Bildung

4 Auswahl und Priorisierung von Zieldimensionen

5 Fazit und Empfehlungen 


\section{Einführung}

Olaf Köller, Johannes Magenheim, Uwe Pfenning, Jörg Ramseger, Mirjam Steffensky, Christian Wiesmüller, Esther Winther, Bernd Wollring

Die Auseinandersetzung mit der Frage, welche Kompetenzen MINT-Multiplikatorinnen und Multiplikatoren in der Weiterbildung pädagogischer Fach- und Lehrkräfte benötigen, ist durch die Schwierigkeit gekennzeichnet, dass es nur wenige wissenschaftlich fundierte Erkenntnisse zur professionellen Kompetenz in den relevanten Fächern und der Professionalisierung frühpädagogischer Fachkräfte gibt. Beispielsweise ist unklar, wie viel Fachwissen oder fachdidaktisches Wissen pädagogische Fachkräfte oder Grundschullehrkräfte benötigen, um mit jüngeren Kindern bereichsspezifische kognitiv anregende Interaktionen umzusetzen. Daraus entsteht die Schwierigkeit, zu benennen, welche Kompetenzen in welchem Ausmaß Multiplikatorinnen und Multiplikatoren für effektive bereichsspezifische Fort- und Weiterbildungen benötigen. So kann man zwar auf umfangreiche Erkenntnisse zur (bereichsunspezifischen) Erwachsenenbildung zurückgreifen, dies gilt aber nicht hinsichtlich der bereichsspezifischen Aspekte (siehe Kapitel 2 Theoretischer Rahmen). Hier wurde in erster Linie plausibilitätsbasiert vorgegangen und auf Erkenntnisse aus der Lehrkräfteprofessionsforschung zur (bereichsspezifischen) professionellen Kompetenz und deren Entwicklung in Fortbildungen zurückgegriffen.

Die vorliegenden Empfehlungen zielen auf MINT-Multiplikatorinnen und Multiplikatoren ab (wie etwa die Trainerinnen und Trainer, die in der Initiative „Haus der kleinen Forscher“ als Weiterbildnerinnen und Weiterbildner tätig sind). Die Frage, ob MINT als ein integrierter Gesamtbereich zu verstehen ist und, wenn ja, was MINT als integrierten Gesamtbereich ausmacht oder ob es doch eher eine Sammelbezeichnung der Fächer ist, die eine besonders hohe wirtschaftliche Bedeutung haben, wird und wurde auch von den Autorinnen und Autoren dieser Expertise kontrovers diskutiert. Aus einer fächerübergreifenden Perspektive wurden vor allem Teile des epistemologischen Wissens als integrale Bestandteile identifiziert. Weiterhin wurde davon ausgegangen, dass Multiplikatorinnen und Multiplikatoren in einem der Bereiche Mathematik, Informatik, Naturwissenschaften (belebte und unbelebte Natur) und Technik vertiefte Erkenntnisse und in den anderen Bereichen sowie dem übergeordneten MINT-Bereich grundlegende Kenntnisse benötigen. Dies folgte der Rationale, dass sich wirkungsvolle Fortbildungen auf ein konkretes Thema (aus einem der vier Bereiche) beziehen. 
In Anlehnung an die Modelle der professionellen Kompetenz von Lehrkräften werden hier das pädagogische Wissen sowie das Fachwissen und fachdidaktische Wissen in den vier MINT-Bereichen skizziert. Die dargestellten Indikatoren der Kompetenz (Könnensbeschreibungen) orientieren sich an dem Niveau, das die Multiplikatoren im Durchschnitt im Laufe ihrer Ausbildung erreichen sollen, wohlwissend, dass Abweichungen in beide Richtungen wahrscheinlich sind. Die Autorinnen und Autoren dieser Expertise sind sich einig, dass letztendlich nur empirische Studien zeigen können, ob sich das vorgeschlagene Kompetenzmodell und die zu den einzelnen Kompetenzen skizzierten Niveaus in der Praxis der Professionalisierung von Multiplikatorinnen und Multiplikatoren bewähren. 


\title{
2 Theoretischer Rahmen
}

\author{
Olaf Köller, Esther Winther
}

\subsection{Professionalisierung des pädagogischen Personals in Kindertagesstätten und Grundschulen}

In den letzten Jahren lassen sich in Deutschland wie auch in vielen anderen Ländern ein verstärktes Interesse sowie ein Ausbau an frühkindlichen Bildungs- und Betreuungssystemen feststellen. Dabei stehen zunehmend bereichsspezifische Bildungsprozesse, vor allem Sprache, Mathematik, Naturwissenschaften und Technik, im Blickpunkt (z. B. Kultusministerkonferenz [KMK] \& Jugendministerkonferenz [JMK], 2004), was sich auch an den Bildungsplänen der Länder ${ }^{4}$ ablesen lässt. Informelle mathematisch-naturwissenschaftliche Erkenntnisprozesse beginnen im Säuglingsalter (Spelke, 1994; Weinert, Doil, \& Frevert, 2008). Eine in Ansätzen systematische institutionelle Anbahnung bereichsspezifischer Kompetenzen fängt in Kitas ab dem vierten Lebensjahr an. Robuste Befunde längsschnittlicher Studien zeigen, dass der Erwerb bereichsspezifischer, grundlegender Kompetenzen (im Sinne von Basiskompetenzen oder Vorwissen) im Elementarbereich eine wichtige Voraussetzung für die spätere schulische Kompetenzentwicklung von Kindern und Jugendlichen darstellt (Anders, 2013; Burger, 2010; National Institute of Child Health and Human Development Early Child Care Research Network [NICHD ECCRN], 2002; Roßbach, 2005; Sylva, Sammons, Chan, Melhuish, Siraj-Blatchford, \& Taggart, 2013). Vor dem Hintergrund der angenommenen Bedeutung der Fachkraft für die kindlichen Bildungsprozesse ist hierbei die Frage der Professionalisierung pädagogischer Fachkräfte im Elementarbereich entscheidend, in Teilen aber auch die der Lehrkräfte im Primarbereich, die beispielsweise in großer Zahl Mathematik fachfremd unterrichten (Richter, Kuhl, Haag, \& Pant, 2012). Auch im Sachunterricht ist die Zahl der Lehrkräfte, die sich selbst als fachlich wenig kompetent bezeichnen, erschreckend hoch: Bei einer Befragung von 555 Lehrkräften aus drei Bundesländern ergab sich, dass fast 40 Prozent der Befragten Sachunterricht oder Naturwissenschaften in der Grundschule unterrichten, ohne eine entsprechende Ausbildung zu haben. Gut ein Viertel der Be-

4 Sammlung aller Bildungspläne unter: http://www.bildungsserver.de/Bildungsplaene-der-Bundeslaender-fuer-die-fruehe-Bildung-in-Kindertageseinrichtungen-2027.html 
fragten (26 Prozent) schätzte die eigene naturwissenschaftliche Qualifikation nur als gering ein (Leser, Mey, Mruck, Ramseger, \& Vock, 2011).

In Abbildung 3 ist ein doppeltes Angebots-Nutzungs-Modell (Kunter, Kleickmann, Klusmann, \& Richter, 2011) aufgeführt, in dem die Kompetenzen der Lernenden (Fachkräfte bzw. Kinder) einerseits von der Qualität der Lerngelegenheiten und andererseits von den individuellen Voraussetzungen abhängen.

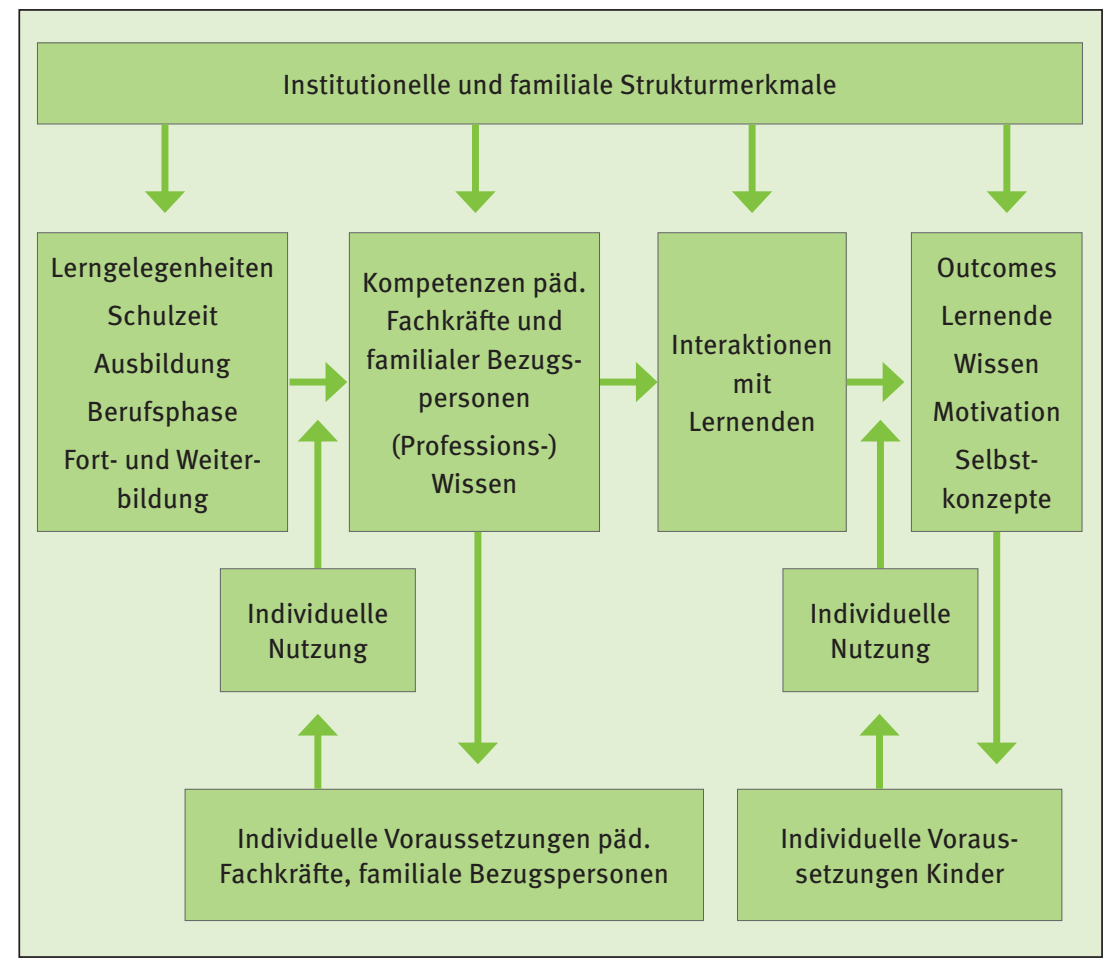

Abbildung 3. Doppeltes Angebots-Nutzungs-Modell in Anlehnung an Kunter, Kleickmann, Klusmann, \& Richter (2011)

Im Angebots-Nutzungs-Modell wird die professionelle Kompetenz der Fachkraft als entscheidend für die Qualität von Lerngelegenheiten und die Entwicklung von Lernenden angenommen. So werden etwa Wissen, Fähigkeiten und Fertigkeiten, die Wahrnehmung und Analyse pädagogischer Situationen sowie die Motivation als zentrale Komponenten professioneller Kompetenz pädagogischer Fachkräfte definiert. Als übergeordnete denk- und handlungsleitende Grundstruktur wird die professionelle Haltung beschrieben, die das Selbst- und Rollenverständnis, motivationale Orientierungen, Werthaltungen und Einstellungen umfasst. 
Empirische Studien aus dem Bereich der Mathematik weisen darauf hin, dass das fachbezogene Professionswissen bzw. die handlungsnahen Kompetenzen pädagogischer Fachkräfte nur schwach ausgeprägt sind (Blömeke, Jenßen, Dunekacke, Suhl, Grassmann, \& Wedekind, 2015). Zudem finden sich große Unterschiede zwischen den Fachkräften.

Für den Primarbereich zeigen die Befunde der internationalen Untersuchung TEDS (Teacher Education Study, Blömeke, Kaiser, \& Lehmann, 2010), dass auch bei Grundschullehrkräften im Fach Mathematik mit substanziellen Defiziten im Professionswissen zu rechnen ist. Da Naturwissenschaften und Naturwissenschaftsdidaktiken ebenso wie die Bereiche Technik und Informatik in der Ausbildung pädagogischer Fachkräfte für den Elementarbereich lediglich eine geringe Rolle spielen, liegt die Vermutung nahe, dass auch ihr Professionswissen in diesen Bereichen eher gering ist bzw. lediglich den Status einer - zudem oft freiwillig erworbenen - Zusatzqualifikation hat. Empirische Befunde fehlen hier aber ebenso wie im Primarbereich.

Das Modell in Abbildung 3 gibt in seinem linken Teil darüber Auskunft, wie professionelle Kompetenzen über die unterschiedlichen Lerngelegenheiten aufgebaut werden. Es beginnt mit fachspezifischen Lerngelegenheiten in der Schule und setzt sich dann berufsspezifisch in der Ausbildung und in der Ausübung der Tätigkeit fort. Zusätzliche Lerngelegenheiten bieten sich durch Fortbildungsveranstaltungen. Deren Wirksamkeit ist im Bereich der Kitas kaum erforscht, wohl aber existieren umfangreichere Arbeiten im Bereich der Wirksamkeit von Lehrkräftefortbildungen. Zudem lassen sich aus Arbeiten im Bereich der Erwachsenenbildung Kompetenzanforderungen für Lehrende identifizieren und Schlussfolgerungen ableiten, unter welchen Bedingungen Fort- und Weiterbildungsmaßnahmen nachhaltig positive Effekte auf ihre Adressatinnen und Adressaten haben. Auf beide Perspektiven - Lehrkräftefortbildung und Erwachsenenbildung - wird im Folgenden eingegangen. In einer Synthese beider Linien werden dann Schlussfolgerungen für die Professionalisierung des Fortbildungspersonals im Bereich der frühen Bildung abgeleitet und Ziele der Professionalisierung von Trainerinnen und Trainern aufgelistet.

\subsection{Merkmale erfolgreicher Lehrkräftefortbildungen}

Die wichtigsten deutschsprachigen Arbeiten zu Effekten erfolgreicher Fortbildungsangebote für Lehrerinnen und Lehrer stammen von Lipowsky (siehe u. a. Lipowsky \& Rzejak, 2015a, 2015b), spezifische Aspekte der Wirksamkeit von Multiplikatorenschulungen werden darüber hinaus in den Arbeiten des Deutschen Zentrums für Lehrerbildung Mathematik (DZLM; siehe u. a. Barzel \& Selter, 2015) adressiert. Erfolg lässt sich dabei auf unterschiedlichen Ebenen festmachen: 
Ebene der Lehrkraft: Ist diese zufrieden mit der Fortbildung? Hat sie etwas gelernt? Haben sich Einstellungen, Kognitionen, Kompetenzen verändert?

- Ebene des Unterrichtshandelns: Hat sich das konkrete Verhalten der Lehrkräfte im Unterricht verändert?

- Ebene der Schülerinnen und Schüler: Lernen die Schülerinnen und Schüler als Folge der Fortbildung erfolgreicher im Fach? Wird ihre Motivation besser gefördert? Verändern sich ihre fachbezogenen Einstellungen?

Da Fortbildungen in erster Linie personenbezogen ausgerichtet sind, wird die Ebene der Institution hier nicht einbezogen. Die Frage nach möglichen Multiplikatoreneffekten durch fortgebildete Fach- und Lehrkräfte innerhalb ihrer Institution sowie die resultierende Organisationsentwicklung wäre ebenfalls zu berücksichtigen, wenn Fortbildungen gezielt derartige Effekte beabsichtigen. Lipowsky und Rzejak (2012) präsentieren mit Blick auf die drei oben genannten Zielebenen sieben Merkmale erfolgreicher Lehrkräftefortbildungen:

- Dauer von Fortbildungen: Längere Fortbildungszeiten scheinen eine notwendige, aber keine hinreichende Voraussetzung für eine erfolgreiche Maßnahme zu sein. Längere Fortbildungen bieten mehr Gelegenheiten für aktives Lernen und wiederholtes Erproben neuer Handlungsschemata.

- Vertiefung des fachdidaktischen und diagnostischen Wissens und Fokus auf Lernprozesse der Schülerinnen und Schüler: Eine Steigerung des fachlichen Verständnisses für die Unterrichtsinhalte (content focus) und mehr Wissen über Konzepte und Misskonzepte von Schülerinnen und Schülern (assessment knowledge) hat nachweisbare positive Effekte auf die oben genannten Zielebenen.

- Wirkungen eigenen Handelns erfahrbar machen: Lehrkräfte sind dann motivierter, Fortbildungsinhalte vertieft zu verarbeiten und sich um einen Transfer in die Unterrichtspraxis zu bemühen, wenn sie erleben, dass sich ihr unterrichtliches Handeln verändern lässt und wenn sie wahrnehmen, dass sich mit diesen Veränderungen auch Veränderungen bei den Schülerinnen und Schülern einstellen.

- Verschränkung von Input-, Erprobungs- und Reflexionsphasen: Professionalisierungsmaßnahmen, die sich mit wiederkehrenden Angeboten auf einen längeren Zeitraum erstrecken, erlauben zwischenzeitlich die Erprobung des Gelernten und die Reflexion, ob das Gelernte hilfreich für die Praxis ist. 
- Orientierung an Merkmalen lernwirksamen Unterrichts: Fortbildungsmaßnahmen, die Bezug zur wissenschaftlichen Literatur nehmen und sich dabei auf die Merkmale lernförderlicher Interaktionsmerkmale konzentrieren, zeigen typischerweise positive Effekte.

- Feedback an Lehrkräfte: Die Rückmeldung zum eigenen Handeln der Lehrkräfte kann durch Coaches, aber auch durch die eigenen Schülerinnen und Schüler erfolgen, beide Formen des Feedbacks sind verhaltensverändernd und fördern die Leistungsstände der Schülerinnen und Schüler.

- Professionelle Lerngemeinschaften: Diese zeichnen sich durch geteilte Werte und Normen, Fokussierung auf das Lernen der Schülerinnen und Schüler, einen reflexiven Dialog, Offenheit und intensive Kooperation aus.

Basierend auf Lipowskys Arbeiten hat das Deutsche Zentrum für Lehrerbildung Mathematik (Barzel \& Selter, 2015) sechs Gestaltungsprinzipien für die erfolgreiche Aus- und Fortbildung von Multiplikatorinnen und Multiplikatoren vorgeschlagen, von denen wir glauben, dass man sie vollständig auf die Aus- und Fortbildung von Trainerinnen und Trainern im Elementarbereich übertragen kann:

- Kompetenzorientierung: Organisatorische, methodische und didaktische Entscheidungen sollten an den inhaltlichen und methodischen Kompetenzen orientiert werden, die Teilnehmende erwerben sollen.

- Teilnehmerorientierung: Vorwissen, Erfahrungen und Bedürfnisse der Teilnehmenden sollten erhoben und daran angeknüpft werden.

Lehr-Lern-Vielfalt: Input-, Erprobungs- und Reflexionsphasen sollten miteinander verknüpft werden.

- Fallbezug: Alltagssituationen im Unterricht bzw. in der Fortbildung sollten als Ausgangspunkt für die Erarbeitung von Grundlagen dienen.

- Kooperationsanregung: Kooperation in Kleingruppen bzw. Lerngemeinschaften sollten als Lern- und Veränderungsanlass genutzt werden.

- Reflexionsförderung: Reflexion über neue Inhalte und die eigene Unterrichtsund Fortbildungspraxis, z. B. durch die Arbeit an konkreten videobasierten Fällen, sollten angeregt werden. 


\subsection{Merkmale erfolgreicher Erwachsenenbildung}

Gute Weiterbildung fängt bei der Gestaltung der Lernangebote an - und die pädagogische Qualität der Angebote ist aus Sicht der erwachsenen Lernenden ein zentrales Qualitätskriterium. Bisherige Ansätze zur Steigerung der Weiterbildungsqualität setzen jedoch fast ausschließlich auf der Ebene der Organisation an; das eigentliche Lerngeschehen und die Beachtung der Lehrenden bleiben weitgehend unberücksichtigt (Hartz, 2011; Tippelt \& Hippel, 2007).

Dabei stellen diese Lehrenden durch ihre Lernangebotsgestaltung den Grundpfeiler für die Weiterbildungsangebote dar, und von ihnen hängt die pädagogische Qualität dieser maßgeblich ab. Die Professionalität der Lehrenden gilt als zentraler Erfolgsfaktor für die Erwachsenenbildung und wird in ihren Voraussetzungen und Fördermöglichkeiten intensiv diskutiert. In diesem Zusammenhang stell sich nicht nur die Frage nach einer Erfassung und Definition vorhandener und notwendiger Lehrkompetenz, sondern auch nach den Möglichkeiten der systematischen Förderung professionellen Lehrhandelns unter Bezugnahme auf die zumeist heterogenen Wissensbestände und Vorerfahrungen sowie die Arbeitsrealität der Lehrenden.

\subsubsection{Eckpfeiler I: In der Weiterbildung Tätige angemessen qualifizieren}

Die berufliche Sozialisation des lehrenden Personals in der Erwachsenen und Weiterbildung ist im Vergleich zu „klassischen“ Professionen durch eine hohe Heterogenität gekennzeichnet (Martin \& Langemeyer, 2014). Nur eine kleine Gruppe von Lehrenden verfügt über eine grundlegende (erwachsenen)pädagogische Qualifizierung. Für das Gros der Lehrenden beruht ihr Zugang zur Lehrtätigkeit als „Quereinstieg“ hingegen vor allem auf fachlicher Expertise oder beruflicher Erfahrung, sodass ihr Unterricht in der Regel ohne expliziten Rekurs auf speziell pädagogisch fundiertes, didaktisch-methodisches Wissen erfolgt (Wirtschafts- und Sozialforschung [WSF], 2005, Martin \& Langemeyer, 2014; Schöb, Sahlender, Brandt, Fischer, \& Wintermann, 2015).

Dies ist dem Umstand zu verdanken, dass es keine allgemein anerkannte Zulassung zu Lehrtätigkeiten in der Erwachsenenbildung gibt. Zwar gibt es in einzelnen Trägerbereichen (z. B. Volkshochschule oder Sport) und im Bereich der Maßnahmenanerkennung in der beruflichen Weiterbildung (nach Sozialgesetzbuch [SGB] und Akkreditierungs- und Zulassungsverordnung Arbeitsförderung [AZAV]) Vorgaben zu den erforderlichen Qualifikationen des Lehrpersonals, aber diese sind noch immer weit gefasst und ermöglichen Spielräume hinsichtlich der pädagogischen Eignung. 
Vorliegende Studien zur beruflichen und sozialen Lage der Lehrenden in der Weiterbildung belegen zudem, dass die Lehrtätigkeit keine „Übergangstätigkeit“ darstellt. Bei einer durchschnittlichen Beschäftigungsdauer von ca. zehn Jahren scheint eine „Investition“ in die Kompetenzen der Lehrenden sowohl für diese selbst als auch für ihre Auftraggeberinnen und Auftraggeber sinnvoll (Martin \& Langemeyer, 2014; Rosenbladt \& Thebis, 2004; WSF, 2005). Angesichts dieser Befunde verwundert es nicht, dass nach den Daten des wbmonitor (Ambos, Egetenmeyer, \& Scheller, 2008) 47 Prozent der Weiterbildungsanbieter einen Weiterbildungsbedarf im Bereich „erfolgreich lehren“ sehen. Da es sich hierbei um eine Kernaufgabe der Lehrenden handelt, ist diese Zahl alarmierend hoch.

Darüber hinaus berichten viele Lehrenden von einer „autodidaktischen“ Qualifizierung und individuellen Professionalitätsentwicklung (Nittel, 2006). Die fehlende Einbindung in professionelle Gemeinschaften („Dozentenindividualismus“) erschwert den kollegialen, fachlichen und sozialen Austausch - beispielsweise in Form von fallbasierter Unterrichtsberatung. Die Teilnahme an Fortbildungen steht häufig in Konkurrenz zur Kursleitung und damit zur Einkommenssicherung, weswegen Angebote, die hohe Anforderungen an Präsenzzeiten stellen, in der Regel wenig nachgefragt werden (Kosubek et al., 2009; Martin \& Langemeyer, 2014). Dennoch weist die Gruppe der Lehrenden eine hohe Fortbildungsbereitschaft auf, die mit 28 Prozent in 2012 über dem Durchschnitt aller Erwerbstätigen mit 21 Prozent liegt (Bilger, 2013).

Es fehlt an zuverlässigem Wissen über die professionelle Kompetenz der Lehrenden. Einzelne Studien zum Lehrverhalten bzw. zum Wissen und Können von Kursleitenden (Bastian, 1997; Hof, 2001; Kade, 1990) bieten ein heterogenes Bild zu den Vorstellungen der Lehrenden über erwachsenengerechten Unterricht; deren unterrichtsbezogenen Handlungen und die dafür grundlegenden Kompetenzen werden darin jedoch nicht systematisch untersucht. Weiter bleibt auch offen, wie Lehrende so gefördert werden können, dass sie ihre Lehrtätigkeit über eine möglichst praxisrelevante Weiterentwicklung ihres Wissens und Könnens verbessern können. Hier können die in der empirischen LehrLern-Forschung und der Lehrkräftebildung geführten Diskurse zur „Expertise“ und „Professionalität“ von Lehrenden theoretische Anknüpfungspunkte bieten.

\subsubsection{Eckpfeiler II: Die Besonderheiten des Lernens Erwachsener berücksichtigen}

Das Handeln der Lehrenden bezieht sind in der Regel immer auf erwachsene Teilnehmende, die über ein bereitgestelltes Angebot etwas lernen können. In diesem mikrodidaktischen Handlungskontext geht es im Kern immer um Tätigkeiten, die darauf zielen, Lehr-Lern-Situationen zu gestalten und andere Menschen dabei zu unterstützen, etwas zu lernen, was sie ohne diese Unterstützung nicht lernen 
würden. Dazu gehört immer auch die Option zu prüfen, ob sie dabei erfolgreich waren. Erfolgreiche Erwachsenenbildung sollte sich an den folgenden Ausgangsfragen messen lassen:

Frage 1: Was sind die Ziele von Maßnahmen und Kursen?

Frage 2: Welche Inhalte werden zur Erreichung der Ziele didaktisch umgesetzt?

Frage 3: In welchem Umfang fließen Forschungsergebnisse über die spezifischen Lernprozesse von Erwachsenen in die Gestaltung der Weiterbildungsangebote ein?

Frage 4: Welchen Einfluss nehmen Professionalisierungsdiskurse auf die Rolle der Lehrenden in der Weiterbildung, und inwiefern tragen diese Professionalisierungsdiskurse damit zur Gestaltung der Weiterbildungsangebote bei?

Frage 1 (Was sind die Ziele von Maßnahmen und Kursen?) scheint aus Forschungsperspektive hinreichend beantwortet: Weiterbildung verfolgt in Anlehnung an Heid (2000) folgende Ziele:

- in der grundlegenden Bildung Erreichtes kumulativ fortführen,

— in der grundlegenden Bildung Versäumtes nachholen,

- im Laufe der Zeit überholte Qualifikationen durch neue ersetzen sowie

- durch Weiterbildung Ermöglichtes oder zu Ermöglichendes in der grundlegenden Bildung einsparen.

Die aktuelle Weiterbildungspraxis lässt sich anhand dieser vier Zielkategorien gut normativ beschreiben und in ihrer Angebots- und Zielgruppenstruktur weitgehend abgrenzen. Ein Blick in die Programm- und Angebotsgestaltung macht allerdings auch deutlich, dass eine Reihe der angebotenen Maßnahmen und Kurse neben kompensatorischen Funktionen gleichsam komplementär und wissenserweiternd ausgerichtet sind.

Mit Blick auf Frage 2 (Welche Inhalte werden zur Erreichung der Ziele didaktisch umgesetzt?) ist ein Verweis auf die Fachdidaktiken unerlässlich. In der Erwachsenenbildung erfährt die Fachdidaktik traditionell wenig Aufmerksamkeit. Dies ist bemerkenswert, da auch in der Erwachsenenbildung Lehr-Lern-Prozesse nach Adressatengruppen, nach Handlungsfeldern und nach spezifischen Inhalten auszurichten sind, die - im Fall der Erwachsenenbildung - durch Bezugswissenschaften sowie durch die Notwendigkeit gesellschaftlicher Partizipation begründet sind (siehe u. a. Wigger, 2004). Eine erwachsenenpädagogische Fachdidaktik ist 
überall da plausibel, wo es ihr gelingt, praktisch relevant zwischen den Interessen der Teilnehmenden auf der einen Seite und den Vermittlungsanforderungen der Inhalte auf der anderen Seite zu reflektieren. Ihr Fokus müssten - hier insbesondere unter Berücksichtigung der aktuellen Kompetenzdiskurse - die jeweils sachgebietsspezifisch zu vermittelnden Kompetenzen und Kompetenzstandards sein (Brandt, 2011; siehe Kapitel 3 Zieldimensionen für Multiplikatorinnen und Multiplikatoren früher MINT-Bildung).

Mikrodidaktisch - also bezogen auf die konkrete Gestaltung von Weiterbildungen - ist Frage 3 (In welchem Umfang fließen Forschungsergebnisse über die spezifischen Lernprozesse von Erwachsenen in die Gestaltung der Weiterbildungsangebote ein?) von besonderer Relevanz. Es liegen kaum Forschungsergebnisse vor, die erwachsene Lernende im Hinblick auf Erfolgskriterien gelingender Lehr-Lern-Prozesse beschreiben. Empirische Evidenz fehlt mit Blick auf diese Frage vollständig; Bezugspunkte lassen sich jedoch in den Konstruktivismusdiskursen der Erwachsenenbildung identifizieren (Arnold \& Siebert, 1995; Rotthaus, 2001). Eine Grundannahme dieser Diskurse ist, dass Erwachsene nur unter Anknüpfung an und unter Fortführung eigener „Lernprojekte“ (Holzkamp, 1993) signifikant zu lernen vermögen. Erwachsene Lernende sind dieser Annahme zu Folge „Anschlusslernende“, deren Lernprozesse auf individuelle Erfahrungen bezogen und sinnstiftend sein müssen.

Auf dieser Annahme basierend lassen sich für die Förderung des Lernens Erwachsener folgende Schlussfolgerungen ziehen: Das Spektrum möglicher Inhalte ist in der Regel reduzierter als in vorgelagerten institutionellen Lernprozessen der Schule, Ausbildung und Hochschule. Die Entscheidung für spezifische Inhalte ist jedoch zielgerichteter und die Identifizierung von Lernbedarfen ist stärker an die persönliche Interessenlage gebunden. Zudem belegen die vorliegenden Befunde, dass die Weiterbildungsbereitschaft von den Bildungserfahrungen in Kindheit und Jugend abhängt und eine höhere allgemeine Bildung die Offenheit und Anschlussfähigkeit befördert. Die Gestaltung von Lehr-Lern-Prozessen in der Erwachsenenbildung hebt sich damit vom Erziehungsanspruch vorgelagerter Bildungsprozesse ab und konstituiert sich in weiten Teilen über besondere Herausforderungen, die eine heterogene Lerngruppe mit sich bringt.

Frage 4 (Welchen Einfluss nehmen Professionalisierungsdiskurse auf die Rolle der Lehrenden in der Weiterbildung, und inwiefern tragen diese Professionalisierungsdiskurse damit zur Gestaltung der Weiterbildungsangebote bei?) lässt sich in der aktuellen nationalen sowie internationalen Diskussion zu den Anforderungen an Lernende verorten. Hier dominiert der Kompetenzbegriff, der auch in der Erwachsenenbildung seit längerem Verwendung findet, wenn Kenntnisse, 
Fähigkeiten und Fertigkeiten beschrieben werden, wobei die Systematik und die begriffliche Konsistenz jedoch stark variieren. Mit der zunehmenden Bedeutung des lebenslangen Lernens auf EU-Ebene und der Forderung einer gleichwertigen Anerkennung - vor allem auch informell erworbener - Kompetenzen durch die Bildungspolitik, gehen Versuche einher, Kompetenzmodelle für Lehrende zu entwickeln, die fach- und trägerübergreifend anwendbar sind (z. B. Kompetenzpass für Weiterbildnerinnen und Weiterbildner bei Wiesner, 2009; GRETA-Kompetenzmodell für Lehrende in der Erwachsenen- und Weiterbildung bei Lencer \& Strauch, 2016) bzw. Anschluss an den Europäischen Qualifikationsrahmen bieten (Egetenmeyer \& Strauch, 2009; Kraft \& Seitter, 2008). Die Modelle schlagen Kompetenzklassen und -niveaus zu einer Einordnung von Lernergebnissen vor, thematisieren aber nicht die Frage der möglichen Entwicklung der darin beschriebenen Dimensionen. Sie nehmen auch die konkreten mikrodidaktischen Anforderungen der Lehrenden nicht in den Blick, wie es in der Lehrkräftebildungs- und Expertiseforschung der Fall ist (Baumert \& Kunter 2006; 2011; Bromme, 1992). Allerdings verfolgen sie den Anspruch, Kompetenzanforderungen zu beschreiben, die in der Erwachsenenbildung Akzeptanz finden. Die Kompetenzmodelle stellen damit eine potenzielle Grundlage dafür dar, berufsrelevante Kompetenzen zertifizieren zu können. 


\title{
3 Zieldimensionen für Multiplikatorinnen und Multiplikatoren früher MINT-Bildung
}

\author{
Olaf Köller, Johannes Magenheim, Uwe Pfenning, Jörg Ramseger, \\ Mirjam Steffensky, Christian Wiesmüller, Esther Winther, Bernd Wollring
}

In diesem Kapitel werden die Ausführungen zur Lehrkräfte- und Erwachsenenbildung zusammengeführt, sie münden in ein Modell der professionellen Kompetenz von Multiplikatorinnen und Multiplikatoren früher MINT-Bildung, wie etwa den Trainerinnen und Trainern der Initiative „Haus der kleinen Forscher“. Für verschiedene Bereiche der professionellen Kompetenz Lehrender, die sich aus pädagogisch-psychologischen Erkenntnissen über Lehren, Entwicklung und Lernen in der Kindheit und im Erwachsenenalter zusammensetzt, werden für unterschiedliche Zieldimensionen so genannte Könnensbeschreibungen (im Sinne von Indikatoren der Zieldimensionen für Trainerinnen und Trainer) formuliert.

Professionelle Kompetenz lässt sich zum einen als die Fähigkeit beschreiben, unter „Zuhilfenahme motivationaler, volitionaler und selbstregulatorischer Ressourcen breit gelagerte, wissenschaftlich vertiefte und damit vielfältige, abstrahierte Kenntnisse in konkreten Situationen angemessen anwenden zu können oder umgekehrt betrachtet: in eben diesen Situationen zu erkennen, welche Bestandteile aus dem Wissensfundus relevant sein können“ (Tietgens, 1988, 37). Zum anderen wird professionelle Kompetenz als die Fähigkeit verstanden, einen Gegenstand aus unterschiedlichen Perspektiven zu betrachten und mit widersprüchlichen Wissens- und Urteilsformen umzugehen (Nittel, 2000). Professionalität zeichnet sich somit durch eine situative Kompetenz aus, die gleichermaßen durch eine gut organisierte Wissensbasis, motivationale Stützsysteme und berufliche Erfahrung geprägt ist (Bromme, 1992; Gruber, 2004).

Das mit der professionellen Lehrkompetenz beschriebene handlungsleitende (prozedurale) Wissen setzt sich wiederum aus unterschiedlichen Dimensionen zusammen, die sich wie folgt unterscheiden lassen: fachliches Wissen, fachdidaktisches Wissen, pädagogisch-psychologisches Wissen, Organisationswissen, Beratungswissen sowie das für eine Lehrtätigkeit in der Erwachsenenbildung und Weiterbildung zusätzlich relevante Wissen über das Feld der Weiterbildung (strukturelle, finanzielle, rechtliche Rahmenbedingungen) und bezüglich ökonomischer Fragen der Rentabilität, Vermarktung etc. der Lehrtätigkeit (Schrader \& Goeze, 2016). Hinzu treten analog zu gängigen Modellen professioneller Kompetenzen in der Lehrkräfteforschung (Kunter, Klusmann, Baumert, Richter, Voss, \& Hachfeld, 
2013) nicht-kognitive Merkmale, so dass sich für Lehrende in der Erwachsenenbildung das Modell professioneller Kompetenz in Abbildung 4 ergibt.

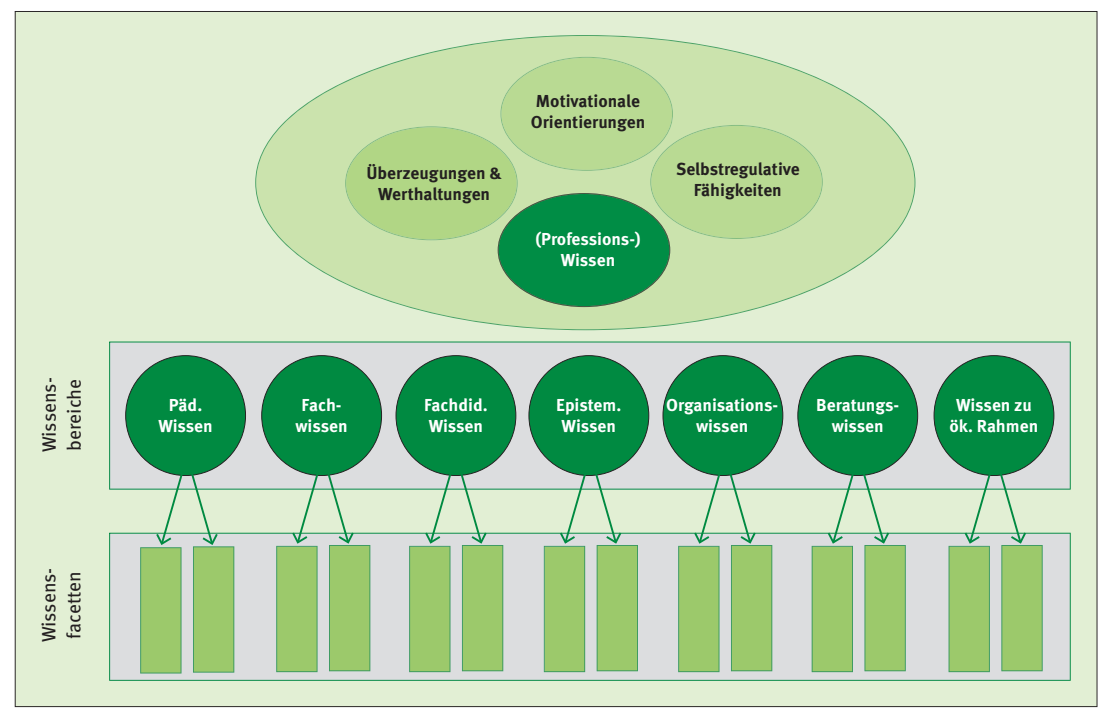

Abbildung 4. Modell professioneller Kompetenz von Lehrenden in der Erwachsenenbildung, basierend auf Kunter, Klusmann, Baumert, Richter, Voss, \& Hachfeld (2013)

Basierend auf dem Modell formulieren wir im Folgenden aus einer erziehungswissenschaftlichen und pädagogisch-psychologischen Perspektive sowie aus einer fach- und fachdidaktischen Perspektive Zieldimensionen und Indikatoren für die Aus- und Weiterbildung von Trainerinnen und Trainern.

\subsection{Motivationale Orientierungen in Bezug auf mathematische, informatische, naturwissenschaftliche und technische Bildung}

Motivationale Orientierungen in Bezug auf MINT-Bildung beinhaltet berufsspezifische Motivation und Enthusiasmus, subjektive Kompetenzüberzeugungen und den Umgang mit berufsbezogenen Konflikt- und Entscheidungssituationen.

Grundlegend für eine nachhaltige Implementierung früher MINT-Bildung in Kitas und Grundschulen ist es, den Trainerinnen und Trainern die Bedeutung der MINT-Bildung in der vorschulischen und schulischen Phase zu veranschaulichen. Sind die pädagogischen Fach- und Lehrkräfte von einer frühen MINT-Bildung überzeugt, bauen sie in sich im Idealfall eine positive, auch reflektierte Einstellung zur MINT-Bildung auf. 
Ausgehend vom Interesse an MINT wird eine positive emotionale Haltung zur MINT-Bildung wahrscheinlich. Die Trainerinnen und Trainer entwickeln hierzu ein positives Selbstkonzept ihrer MINT-bezogenen Fähigkeiten. Ihre Selbstwirksamkeitserwartungen sowie die MINT-bezogene Selbsteinschätzung werden gestärkt. Hierbei spielen die individuellen Zugangsweisen zu den MINT-Disziplinen eine bedeutende Rolle: Sei es durch ein Problem im Alltag, die Faszination an einem Gegenstand oder Phänomen an sich, die Genese der Fachdisziplin, die künstlerische Komponente oder eine begeisternde Geschichte. Diese motivationalen und emotionalen Aspekte unterstützen bei der Umsetzung mathematischer, informatischer, naturwissenschaftlicher und technischer Bildung.

Trainerinnen und Trainer ...

- können ihre emotionale Haltung zu MINT bewerten.

- können ihr Interesse an MINT begründen.

- verfügen über ein positives Selbstkonzept zu MINT.

- können den Stellenwert bzw. die Bedeutung von MINT-Bildung in Kita, Hort und Grundschule erläutern.

- sind bereit, ihr professionelles Selbstverständnis bezüglich Reflexionsfähigkeit, Kooperationsfähigkeit, Entwicklung der Professionalität und Fortbildungsbereitschaft zu analysieren.

- kennen ihre motivationalen Antreiber und können diese auf wissenschaftliche Ansätze der Leistungsmotivationsforschung (Erwartungs-x-Wert-Modelle, Zieltheorien, Volition, Selbstregulation) beziehen.

— können ihre eigenen Fähigkeiten adäquat einschätzen.

- kennen das Konzept der Selbstwirksamkeit und seine Bedeutung für das professionale Handeln.

- kennen motivationsfördernde Rückmelde- bzw. Unterstützungsformate und können diese in der Interaktion sowohl mit Kindern als auch mit Erwachsenen einsetzen.

- kennen die Grundlagen der Interessentheorie.

- können den Enthusiasmus des eigenen professionellen Handelns mit den Leistungen der Adressatinnen und Adressaten in Zusammenhang bringen. 


\section{2 Überzeugungen und Werthaltungen in Bezug auf mathematische, informatische, naturwissenschaftliche und technische Bildung}

Die Überzeugungen und Werthaltungen beziehen sich zum einen auf das professionelle Rollen- und Selbstverständnis als Lernbegleitung und zum anderen auf selbstregulative Fähigkeiten.

Eine positive Haltung der Trainerinnen und Trainer in Bezug auf MINT-Bildung und der Enthusiasmus für die Multiplikationstätigkeit ist wünschenswerterweise die Basis, von der aus die Faszination der einzelnen Disziplinen weitergegeben werden kann. Das dadurch angestrebte professionelle Selbstverständnis MINT-bezogener Bildung ergibt eine weitreichende, themenbezogene Bereitschaft zur Kooperation, Reflexion, Weiterentwicklung der Professionalität und der Fortbildung. Diese Einstellung erleichtert es ihnen, neues Wissen und Können zu erlernen und bestehendes zu vertiefen.

\subsection{1 Überzeugungen und Werthaltungen}

Überzeugungen und Werthaltungen beinhalten pädagogische Werte, Menschenbilder und gesellschaftliche Reflexion, auf die Reflexion des Lehrhandelns, den Umgang mit Feedback und mit berufsbezogenen Konflikt- und Entscheidungssituationen sowie auf die Bedeutung der Weiterbildung.

Trainerinnen und Trainer ...

- können pädagogische Grundhaltungen und ihre Menschenbilder voneinander abgrenzen.

- können Erziehungsstile und inhalte an wissenschaftliche Erkenntnisse und gesellschaftliche Entwicklungen anpassen.

- setzen ethische Werte wie Beachtung der Menschenwürde, Achtung der Menschenrechte, Demokratie, Teilhabe, soziale Gerechtigkeit in der alltäglichen Arbeit um und kennen Vermittlungswege.

- kennen Reflexionsmethoden und verfahren und diskutieren ihre Arbeit im kollektiven Austausch untereinander.

- kennen und nutzen Möglichkeiten der beruflichen Weiterbildung.

- kennen mögliche Konfliktsituationen im praktischen Berufsalltag. 
- kennen motivationsfördernde Rückmelde- bzw. Unterstützungsformate und können diese in der Interaktion sowohl mit Kindern als auch mit Erwachsenen einsetzen.

- kennen Ansprachestrategien für Eltern und Erziehungsberechtigte.

\subsubsection{Selbstregulative Fähigkeiten}

Selbstregulative Fähigkeiten meinen das Bewusstsein über die Rolle als Lehrkraft und ein bewusstes Engagement für die Lernenden im Rahmen der Lehrtätigkeit unter Berücksichtigung der eigenen Ressourcen und professioneller Grenzen.

Trainerinnen und Trainer ...

— interpretieren die Berufsrolle der Fach- und Lehrkräfte im Sinne von Betreuung und Förderung.

- kennen Theorien der Belastungsregulation.

- kennen und nutzen Selbstregulationsstrategien.

- kennen die positiven Auswirkungen des Einsatzes von Selbstregulationsstrategien auf das berufliche Wohlbefinden und die Regulation von Belastung.

\subsection{Fach- und fachdidaktisches Wissen in Mathematik, Informatik, Naturwissenschaft und Technik}

Das Fachwissen in den Disziplinen Mathematik, Informatik, Naturwissenschaften und Technik wird hier differenziert in die nicht immer disjunkten Bereiche inhaltsbezogenes Wissen und prozessbezogenes Wissen, $d$. h. Wissen über die jeweilige Disziplin, das Wissen über Denk- und Arbeitsweisen sowie epistemologisches Wissen (siehe Abbildung 5). Wissen wird hier und im Folgenden als anwendbares, vernetztes Wissen verstanden.

Das inhaltsbezogene Wissen - auch als konzeptuelles Wissen bezeichnet umfasst Wissen über zentrale Ideen, Konzepte, Gesetzmäßigkeiten und Zusammenhänge, während das prozessbezogene Wissen sich auf das Wissen über zentrale Vorgehensweisen bezieht. 


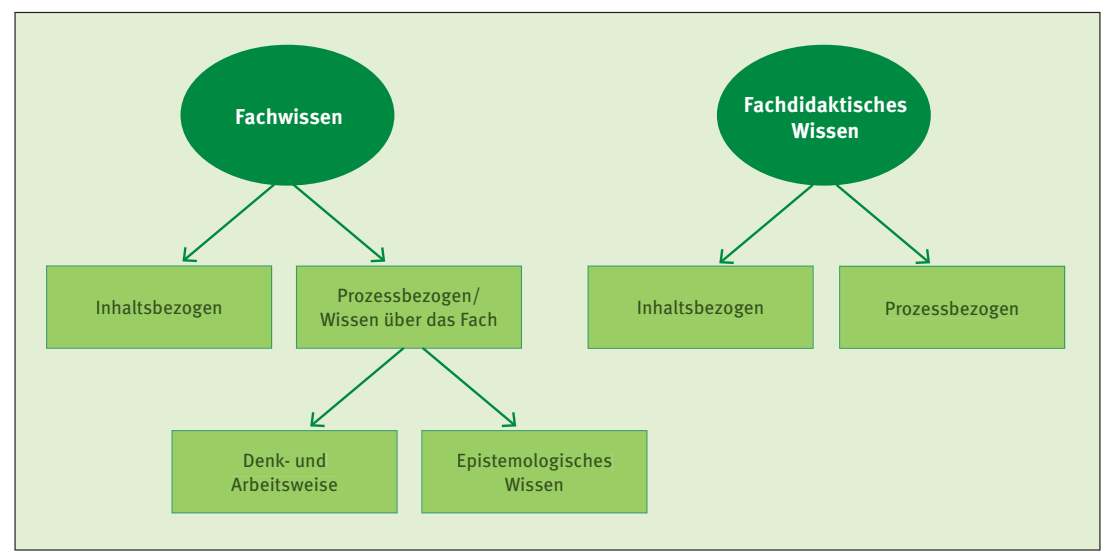

Abbildung 5. Fachwissen und fachdidaktisches Wissen

Der Bereich des prozessbezogenen Wissens oder des Wissens über die jeweilige Wissenschaft umfasst zum einen Wissen über ihre Denk- und Arbeitsweisen. Zum anderen beinhaltet es das Wissen über die Struktur und Genese des Wissens sowie über die Disziplin im gesellschaftlichen Kontext. Teile dieses Wissensbereichs werden - je nach Wissenschaftsmodell - zuweilen als „epistemologische Überzeugungen“ und ab und an als „epistemologisches Wissen“ bezeichnet. Dieses befasst sich mit der Frage, woher die Menschheit etwas weiß und wie Wissenschaftlerinnen und Wissenschaftler in Mathematik, Informatik, Naturwissenschaften und Technik Kenntnisse gewinnen.

Das epistemologische Basiswissen umfasst z. B., dass Naturgesetze nicht „entdeckt“, sondern „formuliert“ werden, dass Mathematik, Informatik, Naturwissenschaft und Technik keine für sich isolierten Disziplinen sind, sondern sich stets in einem spezifischen historischen, kulturellen, gesellschaftlichen und politischen Kontext entfalten und von diesen Kontextbedingungen beeinflusst sind, und dass der Erkenntnisfortschritt in MINT-Disziplinen maßgeblich zur Zivilisation bis zum heutigen Stand geführt hat.

\subsubsection{Mathematisches Fachwissen}

Trainerinnen und Trainer ...

- kennen mathematische Inhalte und ihre Einordnung, insbesondere zu Muster und Strukturen, Raum und Form sowie Zahlen und Operationen.

- kennen Muster und Strukturen als generelle Kennzeichnung der Mathematik und können an Beispielen erzeugende Regeln aufzeigen. 
verfügen über Raumvorstellung und nutzen sie zum Untersuchen, Gestalten und Konstruieren realer geometrischer Objekte.

- verfügen über Zahlensinn im Sinne elementarer Arithmetik und nutzen standardisierte Verfahren ebenso wie flexible Strategien zu Grundrechenarten und zur Proportionalität.

— kennen Problemlösen als genuine und zentrale mathematische Tätigkeit.

- können Beispiele entfalten und dabei Routineprobleme mit vorhandenem Lösungsmuster von Problemen ohne bekannte Lösungsmuster unterscheiden.

— können Strategien zum Problemlösen entwerfen und nutzen.

- kennen Muster und Strukturen als kennzeichnend für die Mathematik, sowohl in Objekten als auch in Prozessen, und können dies an Beispielen entfalten, die für pädagogisches Fachpersonal und Kinder zugänglich sind (siehe auch Benz, Grüßing, Lorenz, Selter, \& Wollring, 2017).

\subsubsection{Informatisches Fachwissen}

Trainerinnen und Trainer ...

- kennen zentrale Konzepte der Informatik wie Daten, Information, Algorithmus, Programm, Automat und Informatiksystem und können diese in Bezug setzen zu ausgewählten, informatischen Inhalten, die für die Kita bedeutsam sind.

- können ausgewählte Phänomene oder Inhalte der Informatik beschreiben und mit ausgewählten, für die frühe informatische Bildung geeigneten Informatiksystemen interagieren, sie explorieren und anwenden (siehe auch Bergner, Köster, Magenheim, Müller, Romeike, Schröder, \& Schulte, 2018).

Inhalts- und Prozessbereiche, die vielen Curricula zur informatischen Bildung zugrunde liegen und zu denen Phänomene und Konzepte bekannt sein sollten, sind: 11: Daten und Information, 12: Algorithmen und Programmierung, 13: Sprachen und Automaten, 14: Informatiksysteme, 15: Informatik, Mensch und Gesellschaft; P0: Interagieren und Explorieren, P1: Modellieren und Implementieren, P2: Begründen und Bewerten, P3: Strukturieren und Vernetzen, P4: Kommunizieren und Kooperieren, P5: Darstellen und Interpretieren (Bergner et al., 2018). 


\subsubsection{Naturwissenschaftliches Fachwissen}

Trainerinnen und Trainer ...

- kennen zentrale Konzepte in den Naturwissenschaften wie Materie, Energie, Kennzeichen von Lebewesen, Struktur und Funktion und können diese in Bezug setzen zu ausgewählten naturwissenschaftlichen Inhalten, die für die Kita bedeutsam sind.

- können ausgewählte Phänomene oder Inhalte beschreiben und in Zusammenhang mit Bedingungen der Umgebung setzen (keine Erklärungen mit submikroskopischen Modellvorstellungen) (siehe auch Anders, Hardy, Pauen, \& Steffensky, 2013; Anders, Hardy, Sodian, \& Steffensky, 2013).

Inhaltsbereiche, die in vielen Bildungsplänen Erwähnung finden und zu denen Phänomene und Konzepte bekannt sein sollten, sind:

- Objekte, Materialien, Materialklassen und ihre Eigenschaften (z. B. Masse, Form bzw. Härte, Aggregatzustand), Veränderungen von Objekten und Materialien, Phänomene wie Bewegung, Schall, Licht und Schatten, Magnetismus, Luft und Feuer, Wetter und Jahreszeiten

- Lebenszyklen sowie Lebensräume von Pflanzen und Tieren sowie Bedürfnisse und Angepasstheit von Pflanzen und Tieren,

- Umgang mit natürlichen Ressourcen (z. B. Mobilität, Trinkwasser, Müll, Energie, Nahrungsmittel),

- Grundlagen einer gesunden Ernährung sowie

- Grundverständnis von Hygiene.

\subsubsection{Technisches Fachwissen}

Trainerinnen und Trainer ...

- können sach- und sicherheitsgerecht mit Werkzeugen, Geräten, Maschinen, Systemen sowie Materialien und Hilfsstoffen umgehen und diese benennen.

- entwickeln ein Verständnis für technische Funktionen, Strukturen und Zusammenhänge (etwa bei der Materialauswahl) sowie für Technik im Alltag.

- können durch Analysieren, (Nach-)Erfinden, Planen und Konstruieren technische Problemlösungen generieren und diese für eigene Objekte nutzen.

- erkennen die Verflechtung von Technik und Gesellschaft (Ropohl, 1979). 
- beziehen über die Funktionalität hinausgehende Aspekte der Technik in ihr Verständnis des soziotechnischen Systems mit ein (Muße, Ästhetik, Prestige, Symbolik, Rituale etc.) (siehe auch Kosack, Jeretin-Kopf, \& Wiesmüller, 2015).

\subsubsection{Epistemologisches Wissen}

Trainerinnen und Trainer ...

- wissen, dass Naturgesetze Konstruktionen des menschlichen Geistes sind, die durch Interpretieren von Daten gewonnen werden, und können dies an Beispielen erläutern.

- kennen typische Denk- und Handlungsformen der Mathematik, Informatik, Natur- und Technikwissenschaften (etwa nach Mustern suchen, Vermutungen äußern, Hypothesen bilden).

- wissen, dass Naturwissenschaft sich vorwiegend im Medium der Sprache vollzieht und experimentelles Handeln kein Selbstzweck, sondern nur ein Mittel zum Zweck der Erkenntnisgewinnung oder Erkenntnissicherung ist.

- wissen, dass das Wissen stets nur aktuelle Geltung hat, und können dies an Beispielen aus der Wissenschaftsgeschichte erläutern (etwa angenommene Ursachen von Krankheiten und Kenntnisse zu deren Bekämpfung).

- kennen die historische, kulturelle und gesellschaftliche Bedingtheit der Wissenschaften (Science in context) und können dies an Beispielen wie Umweltschutz erläutern.

- wissen um die Bedeutung der MINT-Fächer für die berufliche und gesellschaftliche Teilhabe.

Eine große Herausforderung bei der Beschreibung des Wissens von Trainerinnen und Trainern, welches als Voraussetzung für wirkungsvolle Weiterbildungen angenommen wird, ist die Frage des Niveaus. Hier stellt sich zum einen das Problem der sehr heterogenen Voraussetzungen von Multiplikatorinnen und Multiplikatoren, zum anderen der mangelnden empirischen Evidenz. In dieser Beschreibung gehen wir von zwei Orientierungspunkten aus:

Zum ersten müssen die Trainerinnen und Trainer über das zu behandelnde Fachwissen verfügen. Das bedeutet, dass sie über Fachwissen zumindest auf Grundschulniveau verfügen, damit die Pädagoginnen und Pädagogen in der Praxis bei den Kindern keine unangemessenen Vorstellungen anregen oder verfestigen (siehe Abbildung 5).

Zum zweiten gehen wir davon aus, dass die Trainerinnen und Trainer Wissen über zentrale Ideen und Vorgehensweisen sowie Charakteristika der Disziplinen 
verfügen müssen, ohne aber ein in die Tiefe gehendes Detailwissen haben zu müssen. Beispielsweise erscheint es sinnvoll, dass die Trainerinnen und Trainer grundlegendes Wissen über das zentrale Konzept der Energie haben (etwa: Energie ist die Fähigkeit eines Systems, Arbeit zu leisten; mit Energie lassen sich Dinge verändern oder antreiben; Energie wird umgewandelt, aber nicht verbraucht; energetische Veränderungen spielen bei fast allen natürlichen und technischen Prozessen eine zentrale Rolle), ohne dass sie unbedingt Wissen über stärker formalisierte Beschreibungen der Energie oder etwa des Erhaltungskonzepts auf einer fachlich vertieften Ebene im Rahmen der Thermodynamik aufweisen müssen. Dieses grundlegende Wissen über übergeordnete Konzepte und Vorgehensweisen wird als hilfreich für die Einordnung neuer Themen und Inhalte angenommen auch wenn es hierfür wenig Evidenz gibt.

\subsection{Fachdidaktisches Wissen in der mathematischen, informatischen, naturwissenschaftlichen und technischen Bildung}

Im Bereich des fachdidaktischen Wissens in den hier relevanten Disziplinen differenzieren wir zwischen drei Bereichen: Zunächst sollen hier die zwei in der Literatur recht verbreiteten, sehr inhaltsspezifischen Facetten Wissen über Lernendenkognitionen und Unterstützung von Bildungsprozessen genannt werden.

Zum ersten Bereich gehört Wissen über typische Vorstellungen von Kindern zu bestimmten Themen der verschiedenen Disziplinen, etwa „Alles was leicht ist, schwimmt“, oder Wissen über Schwierigkeiten von Kindern beim Erlernen spezifischer Inhalte oder Fähigkeiten, etwa dem simultanen Erfassen von Mengen. Wissen hierüber ist notwendig, um die individuellen Voraussetzungen von Lernenden erkennen und sie hinsichtlich ihrer Anschlussfähigkeit analysieren und die Unterstützung von Bildungsprozessen an diese Voraussetzungen anpassen zu können. Spezifisch für die Mathematik ist in diesem Bereich auch ein grundlegendes Wissen über typische Entwicklungsverläufe, deren Abweichungen sowie Möglichkeiten der Diagnose und Förderung. Anders als in den anderen Disziplinen gibt es in der Mathematik Befunde zur Entwicklung auch zur Gruppe von Risikokindern.

Zur Unterstützung von Bildungsprozessen, dem zweiten Bereich, gehört Wissen über geeignete Beispiele, Alltagssituationen, Spiele, Hands-on-Aktivitäten, die sich für Kinder verschiedener Altersstufen und mit heterogenen Voraussetzungen eignen, um fach- und inhaltsspezifische Bildungsprozesse zu unterstützen, sowie Wissen über die Interaktionsqualität, etwa Wissen über Indikatoren von Interaktionsqualität. 
Zusätzlich gehört zum fachdidaktischen Wissen der Multiplikatorinnen und Multiplikatoren ein stärker übergeordnetes Wissen, das nicht spezifisch für einzelne Inhalte ist. Hierzu zählt auch Wissen über die Ziele der mathematischen, informatischen, naturwissenschaftlichen und technischen Bildung. Eine Einschätzung des angestrebten Wissens der Kita- und Grundschulkinder ist wichtig, um Inhalte nicht vorwegzunehmen und Kinder nicht zu über- oder unterfordern. Beispielsweise wird in den Naturwissenschaften und der Technik in der Regel ein erstes Zusammenhangswissen angestrebt, im Sinne von Wenn-dann- oder Jedesto-Beziehungen von beobachtbaren Variablen, z. B. „Immer wenn es regnet, sind Wolken da“ oder „,e höher ich die Rampe baue, desto weiter rollt die Kugel“. Hierbei handelt es sich im wissenschaftlichen Sinne noch nicht um Erklärungen, für die man häufig abstraktere Modelle benötigt, z. B. Teilchenmodelle oder auch spezifische mathematische Kenntnisse, die in der Regel erst in der Sekundarstufe behandelt werden. Die Erkenntnisse jüngerer Kinder sind in der Regel stärker an spezifische Kontexte gebunden, während ältere Kinder durch vielfältigere Erfahrungen zu allgemeineren Regeln oder Erkenntnissen gelangen können. Weitere Aspekte dieses übergeordneten fachdidaktischen Wissens sind Kenntnisse über die Umsetzungen früher MINT-Bildung in der Praxis, Herausforderungen dabei und auch spezifische Ansätze der kontextgebundenen Sprachförderung.

\subsubsection{Fachübergreifendes fachdidaktisches Wissen}

Trainerinnen und Trainer ...

- kennen zentrale Zieldimensionen früher MINT-Bildung.

- kennen entwicklungs- und kognitionspsychologische Voraussetzungen von Bildungs- und Lernprozessen jüngerer Kinder (Arbeitsgedächtnis, Präkonzepte, Conceptual Change, Vernetzen von Erfahrungsbereichen).

- kennen Ansätze zur Förderung früher (MINT-)Bildung, etwa Lernwerkstätten, Forschungsecken, freies Explorieren, Experimentieren sowie Konzepte der Situationsorientierung und alltagsorientierten Förderung.

- kennen Qualitätsmerkmale von Lernumgebungen (Instructional support, kognitive Aktivierung im Wechselspiel von Ko-Konstruktion und Instruktion, Lernunterstützung, Scaffolding, Sustained Shared Thinking).

- kennen Instrumente zur Erfassung der Interaktionsqualität zwischen pädagogischen Fachkräften und Kindern.

- kennen Möglichkeiten der Organisation adaptiver Lernumgebungen in inklusiven Settings.

- kennen Gemeinsamkeiten und Unterschiede der MINT-Disziplinen. 


\subsubsection{Mathematisches fachdidaktisches Wissen}

Trainerinnen und Trainer ...

- erkennen in der Spiel- und Lebenswelt von Kindern mathematikhaltige Situationen und können diese mit Worten, Bildern oder Gegenständen beschreiben.

- erkennen und fördern mathematisches Verhalten, nehmen Argumentationskeime bei Kindern und Erwachsenen in Handlungsmustern und in der Sprache wahr und unterstützen deren Entfaltung.

- können Sinn, Schönheit und Nutzen von Mathematik auch Kindern und Erwachsenen nahebringen, die ein distanziertes Verhältnis zur Mathematik zeigen.

\subsubsection{Informatisches fachdidaktisches Wissen}

Trainerinnen und Trainer ...

- kennen wichtige fachdidaktische Konzepte von ,Informatischer Bildung“ (etwa fundamentale Ideen der Informatik, „CS unplugged“, „Informatik im Kontext“).

- kennen die begriffliche Vernetzung von informatischen Konzepten und wesentliche Grundvorstellungen über das Fach Informatik.

- können informatische Sachverhalte in verschiedenen Anwendungsbezügen und Sachzusammenhängen aus der Erfahrungswelt der Kinder identifizieren und sie in geeignete Lernsituationen für Kinder integrieren.

- können unter Berücksichtigung der verwendeten methodischen und medialen Arrangements geeignete Bewertungsschemata für informatische Lernsituationen erstellen.

- können in einer angemessenen Sprache über Konzepte informatischer Bildung und deren praktische Umsetzung kommunizieren. 


\subsubsection{Naturwissenschaftliches fachdidaktisches Wissen}

Trainerinnen und Trainer ...

- kennen typische Vorstellungen von Kindern zu ausgewählten, für die Kita und die Grundschule relevanten Inhalten.

- schätzen ein, ob Vorstellungen anschlussfähig oder hinderlich sind.

- kennen Alltagssituationen, in denen ein Phänomen sichtbar wird und die sich für die Beschäftigung mit einem Inhalt eignen.

- kennen Aktivitäten, die sich für die Beschäftigung mit einem Inhalt eignen.

- können Aktivitäten auf deren Eignung für die Unterstützung von Lernprozessen kritisch einschätzen (etwa, wenn sie zu viel Vorwissen erfordern).

- können Voraussetzungen von Lernprozessen in bestimmten Inhaltsbereichen beschreiben (Sequenzierung von Inhalten).

\subsubsection{Technisches fachdidaktisches Wissen}

Trainerinnen und Trainer ...

- können die Bildungsperspektiven bei der Technikvermittlung unterscheiden (Sach- und Strukturperspektive, Handlungsperspektive, Beurteilungs- und Bewertungsperspektive, vorberufliche Orientierung).

- kennen die Medien und Bedingungen für das Raumarrangement bei der Technikvermittlung (Werkzeuge, Ordnungssysteme, Maschinen, Arbeitsplatzgestaltung und Arbeitsorganisation).

- kennen technikspezifische Methoden wie Lehrgang, Fertigungsaufgabe, Konstruktionsaufgabe, Optimierung, Technische Analyse, Technisches Experiment, Demontage-Remontage etc.

- können die Bewertung des technischen Handelns und technischer Entwicklungen anregen und gesellschaftliche Bezüge aufzeigen.

- kennen kulturprägende technikhistorische Entwicklungsverläufe (z. B. Trage, Wagen, Automobil). 


\subsection{Allgemein-pädagogisches Wissen, Beratungswissen und Organisationswissen}

Allgemein-pädagogisches Wissen umfasst die Bildung und Erziehung im frühkindlichen, kindlichen und Erwachsenenalter, Didaktik und Methodik, Lernen, Entwicklung und Sozialisation im (frühen) Kindes- und Erwachsenenalter, Leistungs- und Lernmotivation, Differenzierung, Integration und Förderung, Diagnostik, Beurteilung und Beratung sowie die Bildungsforschung. Beratungswissen meint vor allem Beratung im Sinne von Kommunikation. Organisationswissen bezieht sich zum einen auf Organisationsentwicklung und zum anderen auf Beruf und Rolle der Erzieherin bzw. des Erziehers oder der Lehrkraft im Grundschulbereich. Diese drei Zieldimensionen orientieren sich zum einen an den Standards der Kultusministerkonferenz für die Lehrerbildung (KMK, 2004) und zum anderen an den obigen Ausführungen. Die Besonderheit besteht darin, dass diesbezügliches Wissen sowohl für den Umgang mit Kindern im Elementarund Primarbereich als auch für die Auseinandersetzung mit Erwachsenen in der Fortbildung zentral ist.

\subsubsection{Bildung und Erziehung im frühkindlichen, kindlichen und Erwachsenenalter}

Bildung und Erziehung im frühkindlichen, kindlichen und Erwachsenenalter meint die Begründung und Reflexion von Bildung und Erziehung in institutionellen Settings vor dem Hintergrund umfassender Lernendenorientierung und Förderung.

Trainerinnen und Trainer ...

- kennen pädagogische, soziologische und psychologische Theorien der Entwicklung und der Sozialisation von Kindern.

- kennen etwaige Benachteiligungen von Kindern beim Lernprozess und Möglichkeiten der pädagogischen Hilfen und Präventionsmaßnahmen.

- kennen interkulturelle Dimensionen bei der Gestaltung von Bildungs- und Erziehungsprozessen.

- kennen die Bedeutung geschlechtsspezifischer Einflüsse auf Bildungs- und Erziehungsprozesse.

- erkennen Benachteiligungen und realisieren pädagogische Hilfen und Präventionsmaßnahmen.

- beachten die kulturelle und soziale Vielfalt in der jeweiligen Gruppe. 


\subsubsection{Didaktik und Methodik}

Didaktik und Methodik beinhaltet die Gestaltung von Lernumgebungen im Elementar- und Primarbereich und in der Erwachsenenbildung sowie eine starke Bezugnahme auf authentische Lernkontexte, die anschlussfähiges und kumulatives Lernen erlauben.

Trainerinnen und Trainer ...

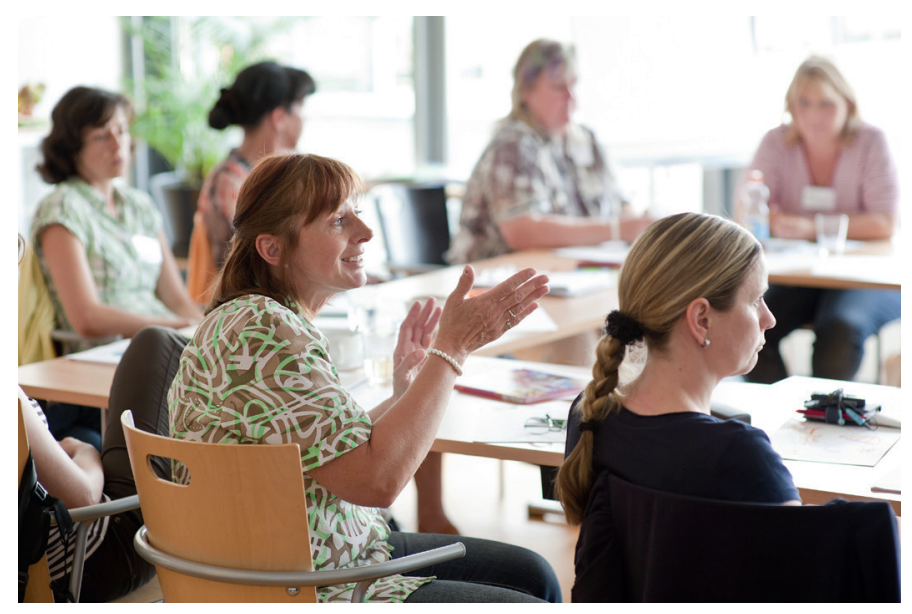

- kennen die einschlägigen Bildungstheorien, verstehen bildungs- und erziehungstheoretische Ziele sowie die daraus abzuleitenden Standards und reflektieren diese kritisch.

- kennen allgemeine Didaktiken und wissen, was bei der Planung von pädagogischen Situationen beachtet werden muss.

- kennen unterschiedliche Unterrichtsmethoden und Interaktionsformen und wissen, wie man sie anforderungs- und situationsgerecht einsetzt.

- kennen Konzepte der Medienpädagogik und -psychologie und Möglichkeiten und Grenzen eines anforderungs- und situationsgerechten Einsatzes von Medien in der Kita bzw. im Unterricht.

- kennen Verfahren für die Beurteilung der Interaktionsqualität bzw. der Lehrleistung und Unterrichtsqualität.

- verknüpfen fachwissenschaftliche und fachdidaktische Argumente und planen gemeinsam mit pädagogischen Fachkräften Lehr- und Lernsituationen, u. a. mit Bezug zu verfügbaren Datenbanken und Wissensangeboten.

\subsubsection{Lernen, Entwicklung und Sozialisation im (frühen) Kindes- und Erwachsenenalter}

Lernen, Entwicklung und Sozialisation im (frühen) Kindes- und Erwachsenenalter bezieht sich auf Lernprozesse von Kindern sowie Erzieherinnen und Erziehern innerhalb und außerhalb von Kindertagesstätten sowie von Schülerinnen und Schülern und Lehrkräften im Grundschulbereich und auf Besonderheiten der Interaktion mit erwachsenen Lernenden. 
Trainerinnen und Trainer ...

- kennen grundlegende Lerntheorien und können diese in spezifischen Situationen anwenden.

- kennen einschlägige Theorien zur körperlichen, kognitiven und sozial-emotionalen Entwicklung.

- kennen relevante Einflussfaktoren individuellen Bildungserfolgs.

- können besondere Ansprüche eines Lernens im Kindesalter von jenen eines Lernens im Erwachsenenalter differenzieren.

- können die institutionellen Zielsetzungen mit den Lernprozessen verknüpfen.

- verstehen Konzepte der Bildungssozialisation und der Bildungsteilhabe.

- kennen Risiken und Gefährdungen des Kindesalters sowie Präventions- und Interventionsmöglichkeiten.

\subsubsection{Leistungs- und Lernmotivation}

Die Leistungs- und Lernmotivation beinhaltet motivationale Grundlagen der Leistungs- und Lernmotivationsentwicklung im Vorschul-, Grundschul- und Erwachsenenalter.

Trainerinnen und Trainer ...

- kennen Grundlagen der Leistungsmotivationsforschung (Erwartungs-x-WertModelle, Zieltheorien, Volition, Selbstregulation).

- kennen die Unterscheidung von intrinsischer und extrinsischer Lernmotivation ebenso wie Grundlagen der Interessentheorie.

- kennen das Konzept der Selbstwirksamkeit.

- kennen die Unterscheidung von individueller und sozialer Bezugsnormorientierung.

- kennen motivationsfördernde Rückmelde- bzw. Unterstützungsformate und können diese in der Interaktion mit Kindern wie Erwachsenen einsetzen. 


\subsubsection{Differenzierung, Integration und Förderung}

Differenzierung, Integration und Förderung bezieht sich auf die Themen Heterogenität und Vielfalt.

Trainerinnen und Trainer ...

- kennen Befunde zum Lernen in heterogenen Gruppen.

- können Methoden der Lernförderung bei unterschiedlichen Leistungsniveaus beurteilen.

- kennen Konzepte der Lern- und Leistungsförderung und wenden diese an.

- verstehen theoretische Konzepte wie Diversität oder Inklusion.

- kennen Ansprachestrategien für Eltern und Erziehungsberechtigte.

\subsubsection{Diagnostik, Beurteilung und Beratung}

Diagnostik, Beurteilung und Beratung umfasst die Diagnose und Förderung individueller Lernprozesse, Leistungsmessungen und Leistungsbeurteilungen sowie Einstellungsbefragungen.

Trainerinnen und Trainer ...

- wissen, wie unterschiedliche Voraussetzungen der Kinder ihre Entwicklungsverläufe beeinflussen.

- kennen Verfahren der Entwicklungsdiagnostik.

- kennen Formen der Entwicklungsverzögerungen und erkennen diese.

- kennen Prinzipien und Ansätze der Elternberatung.

- erkennen Ausgangslagen der Kinder und setzen Fördermöglichkeiten ein.

- kennen Prinzipien erfolgreicher Kooperation zwischen Erzieherinnen und Erziehern sowie Lehrerinnen und Lehrern.

- konzipieren Aufgaben adressatengerecht.

- kennen Prinzipien (Bezugsnormen, Status- vs. Prozessdiagnostik) der Diagnostik.

- kennen erfolgreiche Techniken der Rückmeldung.

- wissen um Methoden, Selbstbilder und Selbstwirksamkeit zu erfassen, d. h. zur Erfassung der Motivation der Zielgruppen. 


\subsubsection{Bildungsforschung}

Die Bildungsforschung bezieht sich auf Theorien, Modelle und Methoden der Bildungsforschung im Bereich frühe Bildung, Entwicklungspsychologie, pädagogische Diagnostik und Erwachsenenbildung.

Trainerinnen und Trainer ...

- kennen Methoden der Fremd- und Selbstevaluation.

- rezipieren und bewerten Ergebnisse der Bildungsforschung (frühe Bildung ebenso wie Schulforschung und Forschung zur Erwachsenenbildung).

- nutzen Ergebnisse der Bildungsforschung für die eigene Tätigkeit.

- kennen und nutzen Unterstützungsmöglichkeiten für Erzieherinnen und Erzieher sowie Lehrerinnen und Lehrer.

- kennen formelle und informelle, individuelle und kooperative Weiterbildungsangebote.

\subsubsection{Kommunikation}

Kommunikation beinhaltet Wissen über Kommunikation im Allgemeinen sowie über Interaktion und Konfliktbewältigung als grundlegende Elemente der Ausund Fortbildung.

Trainerinnen und Trainer ...

- haben Erfahrung in der kommunikativen Steuerung von Gruppenprozessen.

- verfügen über Kenntnisse zur Kommunikation und Interaktion.

- kennen Regeln der Gesprächsführung sowie Grundsätze des Umgangs miteinander, die in pädagogischen Situationen in der Kita und im Unterricht, in Schule sowie in der Elternarbeit bedeutsam sind.

- analysieren Konflikte und kennen Methoden der konstruktiven Konfliktbearbeitung.

- wenden Strategien und Handlungsformen der Konfliktprävention und -lösung an. 


\subsubsection{Organisationsentwicklung}

Organisationsentwicklung meint das Wissen über die Strukturen und die Entwicklung des Betreuungssystems und die Entwicklung der einzelnen Einrichtung.

Trainerinnen und Trainer ...

- kennen und reflektieren den Auftrag der Kindertagesstätte bzw. Grundschule.

- kennen Bedingungen für erfolgreiche Kooperation zwischen Kita und Grundschule bzw. weiteren Einrichtungen im sozialen Umfeld.

- kennen Verfahren der Organisationsentwicklung und können diese anwenden.

- können Gruppen darin unterstützen, gute Arbeitsergebnisse zu erzielen.

- kennen die rechtlichen und finanziellen Rahmenbedingungen im Elementarund Primarbereich.

- kennen die Grundregeln für kollegiale Zusammenarbeit.

- kennen wesentliche Befunde der Stress- und Belastungsforschung.

- können mit Belastungen umgehen.

\subsubsection{Beruf und Rolle der pädagogischen Fach- und Lehrkräfte}

Beruf und Rolle der pädagogischen Fach- und Lehrkräfte umfasst die Professionalisierung, das Berufsfeld als Lernaufgabe zu verstehen, den Umgang mit berufsbezogenen Konflikt- und Entscheidungssituationen sowie den Umgang mit rechtlichen und finanziellen Rahmenbedingungen.

Trainerinnen und Trainer ...

- kennen die Rahmenbedingungen von Ausbildung und Beschäftigung.

- kennen das Berufsbild und wissen dieses in einen gesellschaftlichen Zusammenhang einzuordnen.

- identifizieren sich mit der Tätigkeit als Multiplikatorin bzw. Multiplikator. 


\section{Auswahl und Priorisierung der Zieldimensionen}

Olaf Köller, Johannes Magenheim, Uwe Pfenning, Jörg Ramseger, Mirjam Steffensky, Christian Wiesmüller, Esther Winther, Bernd Wollring

In den vorigen Kapiteln wurden Zieldimensionen für Multiplikatorinnen und Multiplikatoren in der frühen MINT-Bildung beschrieben. Das ausgeführte Modell professioneller Lehrkompetenz (siehe Abbildung 4) leitet, ausgehend vom aktuellen Forschungsstand über die Wirksamkeit von Lehrkräftefortbildungen sowie über gelingende Erwachsenenbildung, relevante Zieldimensionen ab, die für Fortbildende im Bereich der frühen MINT-Bildung relevant sind. Diese theoriebasierte Zusammenstellung ist komplex und umfangreich; auch sind nicht alle darin enthaltenen Kompetenzbereiche gleichartig für die Anwendung in der Praxis (beispielsweise zur Kompetenzeinschätzung oder zur gezielten Weiterentwicklung einzelner Zieldimensionen) geeignet, so dass eine Auswahl und Gewichtung von Zieldimensionen für die Anwendung des Modells angezeigt ist. Diese Priorisierung durch die Autorinnen und Autoren erfolgt vor dem Hintergrund sowohl theoretisch-wissenschaftlicher als auch praxisorientierter Überlegungen. Handlungsleitend für die Praxisorientierung sind dabei vordergründig die Trainerinnen und Trainer der Initiative „Haus der kleinen Forscher“ als ein relevanter Akteur in der frühen MINT-Bildung innerhalb Deutschlands.

Auf der obersten Ebene des Modells sind vier Zieldimensionen spezifiziert (siehe Abbildung 4): Motivationale Orientierungen in Bezug auf MINT-Bildung, Überzeugungen und Werthaltungen in Bezug auf Lernen und Lehren, selbstregulative Fähigkeiten der Lehrenden sowie das Professionswissen. Im Kanon dieser vier großen Kompetenzbereiche werden das Professionswissen sowie die motivationale Orientierung von Multiplikatorinnen und Multiplikatoren als hoch relevante Zieldimensionen für gelingende Fortbildungen im MINT-Bereich für pädagogische Fachkräfte aus Kita, Hort und Grundschule erachtet. Dem Professionswissen kommt als kognitiver Zieldimension hierbei eine übergeordnete Rolle zu, da die darin enthaltenen Aspekte von Wissen und Können den Kern der Professionalität darstellen (Baumert \& Kunter, 2006). Das Modell professioneller Handlungskompetenz von Lehrenden differenziert die übergeordnete Zieldimension Professionswissen weiter aus. Ihr zugeordnet sind sieben Wissensbereiche, welche wiederum teilweise noch weiter aufgegliedert sind. Auf Ebene dieser Wissensbereiche wer- 
den Fachwissen und epistemologisches Wissen, fachdidaktisches Wissen sowie allgemein-pädagogisches Wissen und Beratungswissen priorisiert (siehe Abbildung 6).

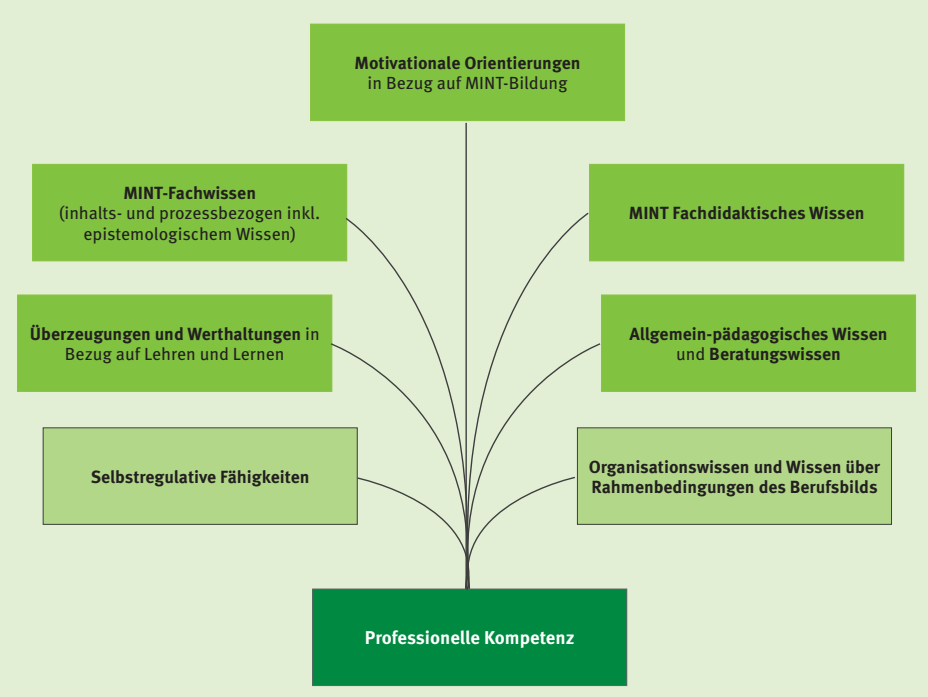

Abbildung 6. Kompetenzbereiche für Multiplikatorinnen und Multiplikatoren früher MINTBildung (vorrangig gewichtete Kompetenzbereiche sind dunkelgrün dargestellt)

\subsection{Fach- und fachdidaktisches Wissen in Mathematik, Informatik, Naturwissenschaft und Technik}

Neben dem allgemein pädagogisch-psychologischen Wissen und Können innerhalb des Professionswissens, sind für Multiplikatorinnen und Multiplikatoren in der frühen MINT-Bildung auch domänenspezifische Wissensbestandteile von Bedeutung. Bei diesen Wissensbestandteilen steht im Gegensatz zum pädagogischen Wissen, das sowohl die Erwachsenen- als auch die Elementar- und Primarbildung umfasst, letzterer Aspekt im Vordergrund. In Anknüpfung an Kapitel 3.3 und 3.4, die die Wissensbereiche Fachwissen und fachdidaktisches Wissen erläutern, wird folgende Gewichtung empfohlen:

Multiplikatorinnen und Multiplikatoren sollten über die MINT-übergreifenden Grundlagen im Fachwissen und fachdidaktischen Wissen verfügen, wie sie die hierzu formulierten Indikatoren näher beschreiben. Domänenunabhängig geht es um das grundlegende Verständnis der (prozessbezogenen) Gemeinsamkeiten der verschiedenen MINT-Fachdisziplinen. Zusätzlich zu diesem Grundverständnis werden vertieftes Fachwissen sowie fachdidaktisches Wissen in mindestens einer 
Domäne für Multiplikatorinnen und Multiplikatoren in der frühen MINT-Bildung als bedeutsam erachtet.

Weiterhin ist das epistemologische Wissen, das auf Wissen über die einzelnen Domänen und die jeweilige Struktur und Genese von Wissen abzielt, in seiner Gesamtheit ebenfalls hervorgehoben. Multiplikatorinnen und Multiplikatoren mit entsprechendem Wissen und Können in diesem Wissensbereich unterstützen dadurch die Fortbildungsteilnehmenden darin, mathematische, naturwissenschaftliche, informatische sowie technische Handlungsweisen in der Auseinandersetzung mit MINT-Phänomenen von anderen zu unterscheiden.

\subsection{Allgemein-pädagogisches Wissen}

Der Wissensbereich allgemein-pädagogisches Wissen (siehe Kapitel 3.5 Allgemein-pädagogisches Wissen, Beratungswissen und Organisationswissen) umfasst die pädagogisch-psychologischen Grundlagen professioneller Kompetenz von Multiplikatorinnen und Multiplikatoren sowohl in Bezug auf das (frühe) Kindesalter als auch auf das Erwachsenenalter und setzt sich im Modell aus insgesamt sieben Wissensfacetten zusammen. Als vorrangig werden davon eingeschätzt:

- Bildung und Erziehung im (früh-)kindlichen und Erwachsenenalter,

- Didaktik und Methodik,

- Lernen, Entwicklung und Sozialisation in der frühen Bildung und im Erwachsenenalter sowie

Differenzierung, Integration und Förderung.

Diese Auswahl betont die Wichtigkeit, die insbesondere in der Gestaltung förderlicher Lernumgebungen unmittelbar im Fortbildungssetting mit Erwachsenen (im Austausch mit Fortbildungsteilnehmenden) und mittelbar mit Kindern (die Fortbildungsteilnehmenden in ihren jeweiligen institutionellen Settings Kita, Hort und Grundschule) liegt. Unabhängig von fachlichen Inhalten und der dazugehörigen Fachdidaktik (siehe Kapitel 4.3 Beratungswissen) ist es wesentlich, dass Multiplikatorinnen und Multiplikatoren dieses allgemeine pädagogisch-psychologische Wissen und Können haben. 


\subsection{Beratungswissen}

Des Weiteren vorrangig gewichtet ist der Wissensbereich Beratungswissen. Kernelement dieses Wissensbereichs ist das Wissen und Können von Multiplikatorinnen und Multiplikatoren, um die Interaktion im Fortbildungssetting angemessen zu gestalten (also die Lernbegleitung). In Bezug auf die Beratungs- und Unterstützungsprozesse sind die Gestaltung der Lernsituation und die Prozessbegleitung der Lernenden entscheidende Faktoren für erfolgreiche Fort- und Weiterbildungsmaßnahmen. Erst die Begleitung und Unterstützung des gesamten Lernprozesses bedingt, dass Merkmale erfolgreicher Fortbildungen, wie sie für Lehrkräfte oder allgemein in der Erwachsenenbildung beschrieben wurden (beispielsweise Feedback an Lehrkräfte oder das Anknüpfen an das Vorwissen und die Vorerfahrungen der Teilnehmenden; siehe Kapitel 3.5 Allgemein-pädagogisches Wissen, Beratungswissen und Organisationswissen), zum Tragen kommen können. 


\section{Fazit und Empfehlungen}

Olaf Köller, Johannes Magenheim, Uwe Pfenning, Jörg Ramseger, Mirjam Steffensky, Christian Wiesmüller, Esther Winther, Bernd Wollring

Fortbildungsangebote für pädagogische Fachkräfte im MINT-Bereich gelten als wichtige Voraussetzung, um die dazugehörigen Bildungsinhalte im Elementarbereich zu implementieren. Für die Gestaltung effektiver Fortbildungsangebote, also solcher, die Wirkungen auf die professionelle Kompetenz der Fachkräfte, die Implementations- und Prozessqualität und darüber vermittelt auf die Kompetenzentwicklung der Kinder zeigen, benötigen die Multiplikatorinnen und Multiplikatoren professionelle Kompetenzen im Bereich der Erwachsenenbildung sowie fachspezifische Kompetenzen, z. B. Wissen über Alltagssituationen, in denen mathematisch-naturwissenschaftliche Ideen erfahrbar werden.

Im Hinblick auf die Professionalisierung der MINT-Multiplikatorinnen und Multiplikatoren besteht die besondere Herausforderung, dass diese sehr unterschiedliche Voraussetzungen mitbringen, die stärker im pädagogischen Bereich und/oder stärker in einem oder mehreren der MINT-Fächer liegen können. Entsprechend müssen passgenaue Angebote für die Multiplikatorinnen und Multiplikatoren entwickelt werden. In den Angeboten sollen nicht nur fehlende Wissensbereiche erarbeitet werden, sondern auch die Entwicklung einer Haltung, die dem pädagogischen Ansatz der Initiative „Haus der kleinen Forscher“ entspricht, angebahnt werden, was dafür spricht, dass entsprechende Angebote in der Regel längerfristig angelegt werden müssen. Für die professionelle Weiterentwicklung erschwerend ist die häufig fehlende Einbindung der Multiplikatorinnen und Multiplikatoren in professionelle Lerngemeinschaften, die dazu führen kann, dass diese sich kaum mit ihren Kolleginnen und Kollegen fachlich austauschen und ihnen entsprechend non-formale Gelegenheiten zur Weiterentwicklung professioneller Kompetenzen fehlen.

In dieser Expertise wurde der Versuch unternommen, grundlegende professionelle Kompetenzen aus den verschiedenen hier relevanten Bereichen zu formulieren. Damit wurden wichtige wissenschaftsbasierte Grundlagen geschaffen, um Zieldimensionen von Fortbildungsangeboten für die Multiplikatorinnen und Multiplikatoren der Initiative „Haus der kleinen Forscher“ zu definieren. Ob das hier beschriebene Kompetenzstrukturmodell und die damit verbundenen Can-do-Beschreibungen sich einerseits als realistisch - d. h. für die Multiplikatorinnen und Multiplikatoren erreichbar - und andererseits als wirksam für die 
Weiterentwicklung des MINT-Bereichs in der Kita erweisen, müssen empirische Untersuchungen zeigen. Wir halten es für dringend geboten, die Niveaus professioneller Kompetenzen der Multiplikatorinnen und Multiplikatoren in einem ersten Schritt im Sinne einer Bestandsaufnahme zu erheben, in einem zweiten Schritt die bestehenden Professionalisierungsangebote für die Multiplikatorinnen und Multiplikatoren damit abzugleichen und ggf. zu erweitern und in einem dritten Schritt die Qualität der Fortbildungen und deren Wirkungen, z. B. auf Überzeugungen sowie das bereichsspezifische Interesse und das professionelle Wissen, zu überprüfen. 


\section{Zieldimensionen für Multiplikatorinnen und Multiplikatoren früher MINT-Bildung aus Sicht einer Bildung für nachhaltige Entwicklung}

Heike Molitor

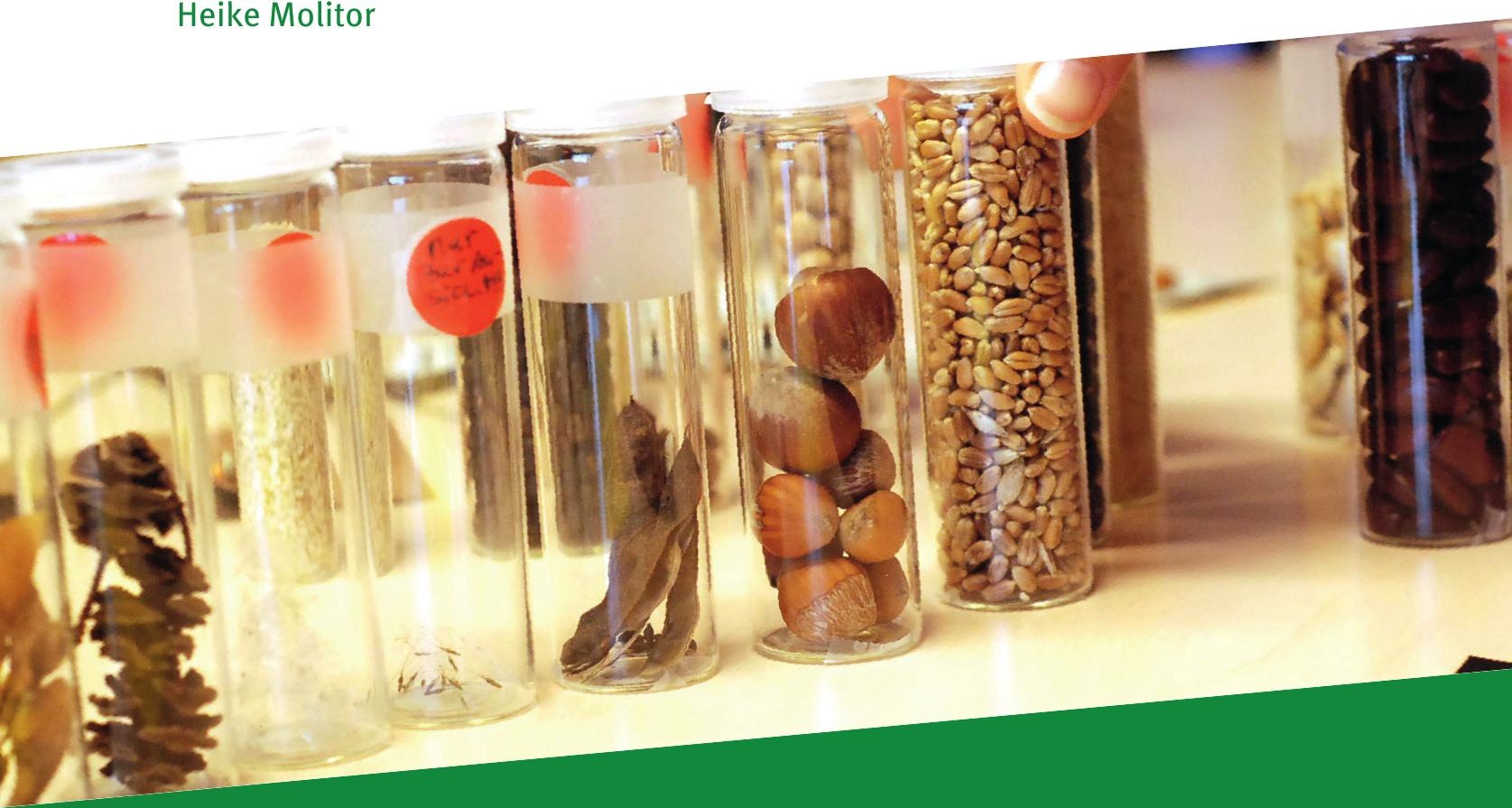

1 Einführung

2 Zieldimensionen einer Bildung für nachhaltige Entwicklung

3 Anforderungen an Multiplikatorinnen und Multiplikatoren einer (MINT-)Bildung für nachhaltige Entwicklung

4 Fazit 


\section{Einführung}

Dieses Kapitel skizziert, wie die Konzepte einer MINT-Bildung und einer Bildung für nachhaltige Entwicklung (BNE) hinsichtlich der Kompetenzen von MINT-Multiplikatorinnen und Multiplikatoren, die als Weiterbildnerinnen und Weiterbildner tätig sind, in ihren Bezügen zueinander fruchtbar gemacht werden können. Damit soll ein Miteinander von Initiativen, Projekten sowie Akteurinnen und Akteuren in beiden „Communities“ unterstützt und gestärkt werden. In dieses Kapitel fließt eine Expertise zu Zieldimensionen einer Bildung für nachhaltige Entwicklung für Kinder, pädagogische Fach- und Leitungskräfte ein (Kauertz, Molitor, Saffran, Schubert, Singer-Brodowski, Ulber, \& Verch, in Vorbereitung).

Mathematik, Informatik, Naturwissenschaft und Technik (MINT) sind keine für sich isolierten Disziplinen, sondern entwickeln sich aus einem spezifischen historischen, kulturellen, gesellschaftlichen und politischen Kontext heraus, von dem sie beeinflusst sind (Kauertz et al., in Vorbereitung) und der auch in der MINT-Bildung bedeutsam ist (Gesellschaft für Didaktik des Sachunterrichts, 2002, 2013). Bildung für nachhaltige Entwicklung setzt sowohl an einem naturwissenschaftlichen Verständnis von Phänomenen als auch an einer sozialwissenschaftlichen Kontextualisierung an, mit dem Ziel einer gesellschaftlichen Transformation in Richtung nachhaltiger Entwicklung. In der Auseinandersetzung von MINT-Anwendung und Nachhaltigkeitsdiskurs entstehen mitunter Spannungsfelder, die sich zwischen einem naturwissenschaftlich begründeten und möglicherweise utilitaristisch geprägten Diskurs (Natur nutzen, Natur zu Eigen machen, Technik optimieren, Technikfolgen ausblenden) und einem normativ begründeten Diskurs über Nachhaltigkeit und veränderte gesellschaftliche Naturverhältnisse ergeben (siehe auch Kauertz et al., in Vorbereitung). Im sozialwissenschaftlichen Diskurs zur nachhaltigen Entwicklung wird davon ausgegangen, dass globale Nachhaltigkeitsprobleme von Menschen verursacht sind und ihnen daher nicht nur mit technologischen oder naturwissenschaftlichen Entwicklungen begegnet werden kann, sondern soziale Veränderungen und Diskurse (bspw. über den Nutzen und die Grenzen bestimmter technologischer Entwicklungen) aktiv eine wesentliche Rolle spielen (Davis, 2012). Neben dieser sozialwissenschaftlichen Expertise stärkt Bildung für nachhaltige Entwicklung die in einer MINT-Bildung schon angelegten Ansätze des situierten Lernens. Insbesondere das partizipative Anknüpfen an Alltagserfahrungen und der Bezug zur Lebenswelt sind hier bedeutsam (siehe auch Kauertz et al., in Vorbereitung). 
Zwischen den Zieldimensionen für Multiplikatorinnen und Multiplikatoren früher MINT-Bildung und den Zieldimensionen einer Bildung für nachhaltige Entwicklung für Kinder, pädagogische Fach- und Leitungskräfte bestehen auf den ersten Blick Unterschiede, aber auch Gemeinsamkeiten und Überschneidungen, z. T. aber mit einer etwas anderen Schwerpunktsetzung. Ein direkter Vergleich ist nicht ganz einfach, da die Zielebenen der beiden Expertisen nicht identisch sind. In diesem Kapitel soll auf die Anforderungen für die Multiplikatorinnen und Multiplikatoren als Weiterbildnerinnen und Weiterbildner in der frühen Bildung fokussiert werden. 


\section{Zieldimensionen einer Bildung für nachhaltige Entwicklung}

Die Zieldimensionen einer Bildung für nachhaltige Entwicklung sind im folgenden Schichtenmodell abgebildet (siehe Abbildung 7). Eine ausführliche Darstellung und Erläuterung findet sich bei Kauertz et al. (in Vorbereitung). Im Zentrum finden sich die Zieldimensionen für Kinder, pädagogische Fachkräfte, die Kitaleitung und die Träger. Diese gelten inhaltlich auch für die Multiplikatorinnen und Multiplikatoren. Sie spielen als Weiterbildnerinnen und Weiterbildner eine wichtige Rolle in der Logik dieses in sich geschachtelten Systems. Die genannten Zielbereiche gelten demzufolge nicht nur für die Kinder, sondern auch für alle Akteurinnen und Akteure der jeweiligen Einrichtung bzw. für die Träger und die Gesellschaft.

Die Zieldimension Motivation beinhaltet Interesse und Neugierde, sich ausgehend vom eigenen Alltag Fragen zu Themenfeldern der Nachhaltigkeit zu stellen. Bei dieser Zieldimension sind auch die Selbstwirksamkeitserwartung und die Auseinandersetzung mit anderen über Nachhaltigkeit bedeutsam.

Unter der Zieldimension Verstehen und Erkennen werden Begriffe und Konzepte verstanden, die für eine nachhaltige Entwicklung relevant sind. Diese umfassen: Mensch-Natur-Verhältnis, Größenordnungen, Verständnis von Zeit (in kleinen und großen Zeiträumen), (Verständnis von) Raum, Mengen und Gruppengrößen, Systeme, Kreisläufe, Natur, Verantwortung für das eigene Handeln, Gemeinschaft und Gesellschaft, Konzept der demokratischen Teilhabe und Vorstellungen von Zukunft.

Die Zieldimension Werte und moralische Optionen umfasst die Reflexion von Werten und die Vorbereitung von Entscheidungen und Handlungen, die (weltweit geteilte) Werte wie Gerechtigkeit, Wertschätzung der Natur und sozialer Vielfalt einbeziehen.

Die Zieldimension Reflektieren und Bewerten beinhaltet die Identifikation von Problemen aus verschiedenen Perspektiven wie Ökonomie, Ökologie, Soziales/Kulturelles und die Einordnung von Informationen bzw. Erkenntnissen im Hinblick auf Nachhaltigkeit.

Unter der Zieldimension Handeln wird eine Entscheidungs- und Handlungsfähigkeit im Sinne einer nachhaltigen Entwicklung verstanden, insbesondere im Treffen von Entscheidungen, in der Handlungsplanung, im Aushandeln verschiedener Lösungen und in der selbstbestimmten Beteiligung an Veränderungen im eigenen Alltag. 


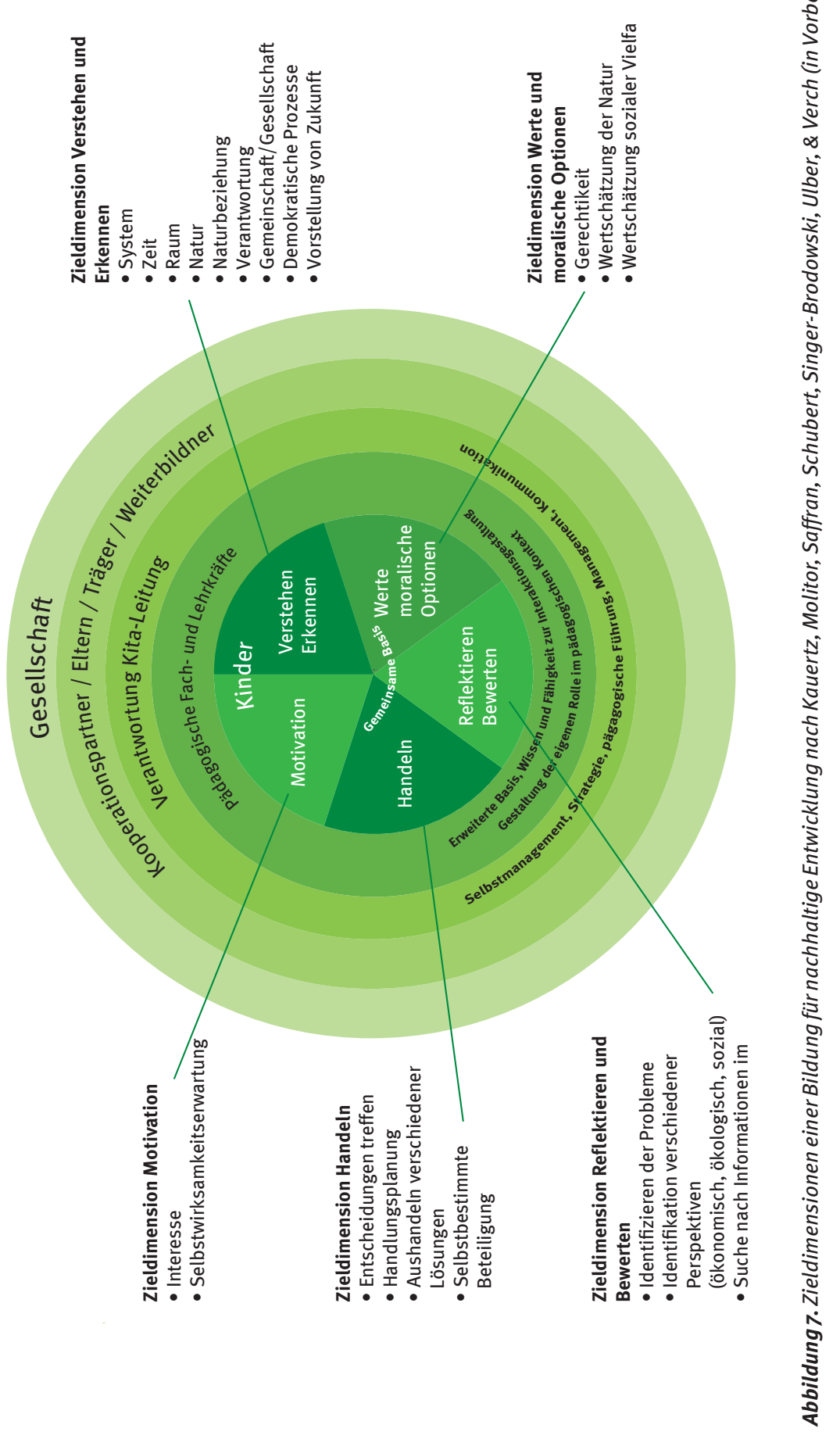




\section{Anforderungen an Multiplikatorinnen und Multiplikatoren einer (MINT-)Bildung für nachhaltige Entwicklung}

Im Vergleich der Zieldimensionen der MINT-Bildung für Multiplikatorinnen und Multiplikatoren und der Zieldimensionen für eine Bildung für nachhaltige Entwicklung fällt auf, dass es Überschneidungen in einer Reihe von Zielbereichen gibt, wie motivationale Orientierungen, Überzeugungen und Werthaltungen, MINT-Fachwissen, MINT Fachdidaktisches Wissen sowie Organisationswissen. Im Folgenden werden diese Zieldimensionen beschrieben und mögliche Erweiterungen aus der Perspektive einer nachhaltigen Entwicklung formuliert (siehe Abbildung 8).

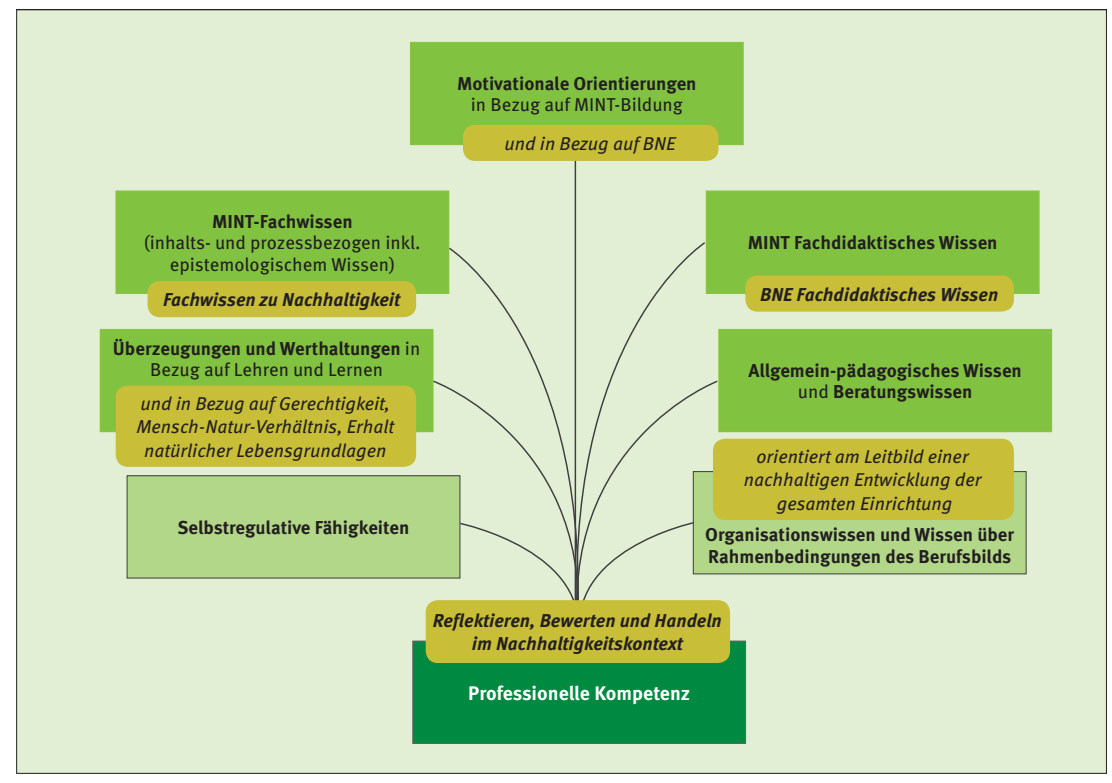

Abbildung 8. Kompetenzbereiche für Multiplikatorinnen und Multiplikatoren der MINTBildung ergänzt um Aspekte einer Bildung für nachhaltige Entwicklung (kursiv in gelben Kästen)

Grundlegend für beide Konzepte sind die motivationalen Orientierungen. Interesse und Begeisterung sowie Selbstwirksamkeitserwartungen sind nicht nur für Multiplikatoren und Multiplikatorinnen in der MINT-Bildung, sondern auch für Multiplikatoren und Multiplikatorinnen einer Bildung für nachhaltige Entwicklung elementar. Bei dieser Zieldimension gibt es folglich einen großen Überschneidungsbereich, der um nachhaltige Entwicklung bzw. Bildung für nachhaltige Entwicklung erweitert wird. 
MINT-Bildung und Bildung für nachhaltige Entwicklung unterliegen aneinander anschlussfähigen professionellen Rollen- und Selbstverständnissen. Dies gilt insbesondere für die Zieldimension der Überzeugungen und Werthaltungen bzw. der pädagogischen Werte. Vor allem ethische Werte wie Menschenwürde, Menschenrechte, Demokratie, Teilhabe und soziale Gerechtigkeit sind in der MINT-Bildung (siehe Kapitel 3.4 Fachdidaktisches Wissen im Abschnitt von Köller et al.) und in einer Bildung für nachhaltige Entwicklung bedeutsam (siehe auch Kauertz et al., in Vorbereitung). In einer Bildung für nachhaltige Entwicklung spielt vor dem Hintergrund zunehmender (globaler) Herausforderungen wie Klimawandel und dessen ökologische, soziale und ökonomische Folgen, weltweite Armut und Ungleichheit, Artensterben u. a. die Frage der Gerechtigkeit eine grundlegende und tragende Rolle. Der Gerechtigkeitsaspekt wird vor diesem Hintergrund zwischen den jetzt lebenden Menschen (globale Gerechtigkeit) sowie zwischen den zukünftig und jetzt lebenden Menschen (Generationengerechtigkeit) in Bezug auf die Befriedigung grundlegender Bedürfnisse differenziert. Darüber hinaus sind das Mensch-Natur-Verhältnis und der Erhalt der natürlichen Lebensgrundlagen in einer Bildung für nachhaltige Entwicklung elementar. Die Reflexion spezifischer Werte und moralischer Normen bildet einen Entscheidungs- und Handlungsrahmen für die Multiplikatorinnen und Multiplikatoren, aus dem sich eine (pädagogische) Haltung entwickeln kann. Diese Haltung beruht auf zentralen Aspekten im Leitbild einer nachhaltigen Entwicklung ergänzt sich mit Werthaltungen, die in der MINT-Bildung diskutiert werden.

MINT-Fachwissen und Wissensbereiche einer nachhaltigen Entwicklung beinhalten als Gemeinsamkeit sowohl mathematisches wie auch informatisches, naturwissenschaftliches, technisches und epistemologisches Fachwissen (siehe Kapitel 3.3 Fach- und fachdidaktisches Wissen im Abschnitt von Köller et al.). Wissen um Nachhaltigkeit umfasst dabei explizit auch gesellschaftliche und ökonomische Wissensbereiche und deren Vernetzung miteinander sowie das Wissen über (gesellschaftliche) Veränderungsprozesse (Kauertz et al., in Vorbereitung).

Für Multiplikatorinnen und Multiplikatoren der MINT-Bildung und einer Bildung für nachhaltige Entwicklung ist es wichtig, über ein angemessenes Fachwissen, über zentrale Konzepte und Vorgehensweisen sowie Charakteristika der Disziplinen und deren interdisziplinäre Verbindung zu verfügen, ohne aber überall ein in die Tiefe gehendes Detailwissen haben zu müssen (siehe Kapitel 3.3 Fach- und fachdidaktisches Wissen im Abschnitt von Köller et al.). So sind beispielsweise Kenntnisse mathematischer Grundformen wie Raum und Zeit nicht nur für MINT-Bildung, sondern auch für eine Bildung für nachhaltige Entwicklung grundlegend (siehe Kapitel 3.3 Fach- und fachdidaktisches Wissen im Abschnitt von Köller et al. sowie Kauertz et al., in Vorbereitung). Während für das MINT-Ver- 
stehen zentrale Konzepte der Naturwissenschaften wie Energie in Bezug zu ausgewählten naturwissenschaftlichen Inhalten mit Bezug zum Alltag der Kinder und der Kitas gesetzt werden, spielen bei einer (MINT-)Bildung für nachhaltige Entwicklung gleichrangig auch sozialwissenschaftliche Inhalte und ethische Fragen eine stärkere Rolle (s. u.). Bei der Bearbeitung des Themas Energie, das für MINT-Bildung und eine Bildung für nachhaltige Entwicklung gleichermaßen relevant ist, ist unter dem Fokus einer nachhaltigen Entwicklung eine Kontextualisierung des Energiethemas um Nachhaltigkeitsaspekte, insbesondere auch um die soziale Perspektive, notwendig. Die Industrieländer als die größten Energienutzer bzw. -verbraucher auf der Welt sind für den größten Anteil am weltweiten $\mathrm{CO}_{2}$-Ausstoß verantwortlich. Das hat vielfältige Folgen, z. B. Klimaerwärmung, Migration und Armut. Eine Reduzierung des Energieverbrauchs durch Effizienzmaßnahmen (technische Innovationen u. a.) und Suffizienz-Strategien (Umgang mit Energie, Lebensstile u. a.) sind daher unabdingbar (Molitor \& Ibisch, 2018).

Für den Elementar- und Primarbereich gilt es, diese komplexen Zusammenhänge didaktisch zu reduzieren und damit verstehbar zu machen, ohne moralische Schuldgefühle bei einer Generation zu wecken, die für die gegenwärtigen Zustände nicht verantwortlich ist. Dies stellt entsprechende Anforderungen an pädagogische Fach- und Lehrkräfte sowie entsprechend an Multiplikatorinnen und Multiplikatoren, die diese weiterbilden. Anschaulich ist der Weg unserer Nahrung vom Acker auf den Teller. Es wird viel Energie aufgewendet für Anbau, Aufzucht, Ernte, Transport, Verarbeitung, Verpackung und Verkauf. Je nach Anbauart, Transportweg, Verarbeitung und Verpackung ist der Energieverbrauch unterschiedlich hoch und die Folgen für die Natur anders. Neben dem Energieverbrauch kann auch die Erzeugung von Energie von erneuerbaren und/oder konventionellen Energieträgern kontextualisiert werden. Greifbar werden diese Themen durch konkrete Anschauung wie eine Solaranlage auf dem Dach der Kita (Rathgeber, 2012; Schubert et al. 2013). Das bedeutet, dass Multiplikatorinnen und Multiplikatoren nachhaltigkeitsrelevante Zusammenhänge an konkreten Konzepten der MINT-Bildung herstellen und diese didaktisch für pädagogische Fach- und Lehrkräfte aufbereiten können. Dafür sollten sie in ihrer eigenen Kompetenzentwicklung unterstützt werden - etwa durch die Qualifizierungsangebote der Stiftung „Haus der kleinen Forscher" für die Trainerinnen und Trainer, die als Multiplikatorinnen und Multiplikatoren in der Initiative fortbildnerisch tätig sind.

Die naturwissenschaftliche Erfahrung der Welt in der frühkindlichen Pädagogik kann durch die Aufnahme einer Nachhaltigkeitsperspektive in Bezug auf das fachdidaktische Wissen durch vielfältige Impulse bereichert werden. Neben dem Forschen, Entdecken, Experimentieren u. a. können narrative (sinnstiftende Erzählungen), ästhetische, ethische, physiomotorische (Aneignung von Natur durch 
Bewegungsvielfalt), partnerschaftliche Zugänge zu den Phänomenen und Themen gesucht werden. „Sich der Natur und Welt zu nähern und sie kompetent zu gestalten, kann vielleicht unter dem didaktischen wie bildungstheoretischen Paradigma des Staunens, Verfremdens, Kontrastierens, von Differenz/Heterogenität als eine verbindende Metapher zwischen MINT-Bildung und einer Bildung für nachhaltige Entwicklung, ein Narrativ, eine Handlungspraxis gesehen bzw. begründet werden“ (Kauertz et al., in Vorbereitung). Das heißt insbesondere auch, dass Zugänge zur Natur, die nicht nur auf objektivierbaren Methoden (beispielsweise eher distanzierten Beobachtungen), sondern auch auf einer subjektiven Herangehensweise (beispielsweise einer emotionalen oder ästhetischen Annäherung) basieren, möglich sind. Dieses Bestaunen und Verstehen kann in vielfältiger bildender Intention geschehen und dabei wesentlich auch die Suche nach neuartigen gesellschaftlichen Naturverhältnissen und Umweltgestaltungen auslösen, durch Beobachtung, Interpretation und Inszenierung oder durch demokratisch orientierte Methoden wie Kinderparlamente. So begeben sich Kinder frühkindlich explorierend in das Entdecken und Erfahren der Welt als kleine Forscherinnen und kleine Forscher (Kauertz et al., in Vorbereitung). Multiplikatorinnen und Multiplikatoren können dies in der Weiterbildung mit den pädagogischen Fach- und Lehrkräften thematisieren und Erfahrungsmöglichkeiten schaffen.

Das Organisationswissen bezieht sich $u$. a. auf die Entwicklung der gesamten Einrichtung im Sinne eines Whole Institution Approach (Ferreira, Ryan, \& Tilbury, 2006). Hier setzt eine Bildung für nachhaltige Entwicklung gezielt an und hat zum Ziel, Nachhaltigkeit als gelebtes Prinzip der Einrichtung für die Lernenden erfahrbar zu machen (Deutsche UNESCO-Kommission, 2014). Ein umfassender Organisationsentwicklungsprozess in Richtung Nachhaltigkeit gestaltet in einem partizipativen Prozess - ausgehend von einer Verankerung im Leitbild - eine Veränderung der Strukturen und Abläufe einer Organisation. Das können beispielsweise die Einführung von Energie- und Wassersparsystemen oder die Umstellung der Essensversorgung auf ein bio-regional-faires Angebot sein (Kauertz et al., in Vorbereitung). Das Handeln in der Organisation wird so einerseits am Leitbild der Nachhaltigkeit ausgerichtet und andererseits zum Lernraum für das pädagogische Arbeiten. Auch für die MINT-Bildungsqualität einer Einrichtung sind eine systemische Perspektive und Organisationswissen als Zieldimensionen für Multiplikatorinnen und Multiplikatoren wichtig (siehe Kapitel 3.5 Allgemein-pädagogisches Wissen, Beratungswissen und Organisationswissen im Abschnitt von Köller et al.). Für die Weiterbildung sind hier neben der Gruppe der pädagogischen Fachund Lehrkräfte insbesondere auch die pädagogischen Leitungen von Bedeutung, welche durch die Multiplikatorinnen und Multiplikatoren im Voranbringen ihrer Einrichtungsentwicklung unterstützt werden können. 
Mit den für eine Bildung für nachhaltige Entwicklung formulierten Zieldimensionen Reflektieren und Bewerten sowie Handeln wird der transformative Charakter dieses Bildungskonzeptes besonders deutlich. Diese transformative Perspektive wirkt bis in die Einrichtung. Im Sinne des Whole Institution Approach soll auch die pädagogische Einrichtung bzw. die Institution (als Ort der Alltagserfahrung) als Lernanlass dienen bzw. zum Lerngegenstand gemacht werden (Deutsche UNESCO-Kommission, 2014). Multiplikatorinnen und Multiplikatoren, die als Weiterbildnerinnen und Weiterbildner eine Einrichtung systemisch in ihrer Entwicklung unterstützen möchten, sehen sich somit einem erweiterten Aufgabenspektrum gegenüber.

Die Zieldimension Reflektieren und Bewerten ist in einer Bildung für nachhaltige Entwicklung durch einen suchenden Lernprozess bzw. eine suchende Lernkultur bei Problemen nachhaltiger und nicht-nachhaltiger Entwicklung insbesondere relevant. Urteile sind nicht per se vorgegeben. Die kritische Distanz und Urteilskompetenz hinterfragt Lösungen und wägt ökologische, ökonomische und soziale Aspekte miteinander ab. Multiplikatorinnen und Multiplikatoren können selber eine Reflexionsfähigkeit für gesellschaftliche Problemlagen entwickeln und ganzheitliche Bildung für pädagogische Fach-, Lehr- und Leitungskräfte ermöglichen. Durch entsprechende Interaktionen ermöglichen sie Reflexionsräume, die Differenzen, unterschiedliche Erfahrungen und Einschätzungen einbeziehen. Wichtig ist auch das Reflektieren des eigenen (beruflichen) Alltags (Kauertz et al., in Vorbereitung). Die Multiplikatorinnen und Multiplikatoren sind auch zur nachhaltigkeitsorientierten Selbstreflexion befähigt.

Auf Grundlage dessen ist ein wesentliches Ziel einer Bildung für nachhaltige Entwicklung die Entwicklung von Entscheidungs- und Handlungsfähigkeit über nachhaltigkeitsrelevante Themen und in nachhaltigkeitsrelevanten Problemfeldern. Multiplikatorinnen und Multiplikatoren haben die Aufgabe, pädagogische Fach-, Lehr- und Leitungskräfte dazu zu befähigen, Experimentier- und Handlungsräume zur Wahrnehmung, Thematisierung und Neuerzeugung von Situationen des nachhaltigen Handelns (in der Bildungseinrichtung) zu eröffnen. Dabei geht es nicht darum, konkrete Handlungen zur Lösung von nachhaltigkeitsrelevanten Problemen vorzuschreiben, sondern grundlegend (und eigenständig) Handlungsdispositionen zu befördern (Kauertz et al., in Vorbereitung). 


\section{Fazit}

MINT-Bildung und Bildung für nachhaltige Entwicklung können in einem konstruktiven Miteinander gegenseitige Reflexionsprozesse anregen. Die Kontextualisierung von Themen in das Feld der nachhaltigen Entwicklung hat eine starke Relevanz für die Lebens- und Arbeitswelt von Menschen. Bildung für nachhaltige Entwicklung hat vor dem Hintergrund globaler Herausforderungen (Klimawandel, Armut, Artensterben etc.) zum Ziel, (gesellschaftliche) Transformationsprozesse in Richtung einer nachhaltigen Entwicklung in Gang zu bringen. Das Leitbild einer nachhaltigen Entwicklung beinhaltet die Forderung nach Gerechtigkeit im Sinne eines guten Lebens für alle Menschen - für die, die jetzt leben, und die, die heute und in Zukunft auf diesem Planeten ihr Leben gestalten. MINT-Bildung ist dafür eine „gute Partnerin“, die in vielen hier beschriebenen Zieldimensionen anschlussfähig ist. Für mögliche Veränderungsprozesse in der Gesellschaft oder aber in der eigenen Kita sind Handlungsdimensionen einhergehend mit einem Reflexions- und Bewertungsprozess unabdingbar. Multiplikatorinnen und Multiplikatoren, die im Kontext einer solchen (MINT-)Bildung für nachhaltige Entwicklung in der Weiterbildung tätig sind, sollten in Bezug auf die oben geschilderten Anforderungen kenntnisreich und handlungssicher sein. 



\section{Fazit und Ausblick - Wie die Stiftung „Haus der kleinen Forscher“ mit den Erkenntnissen umgeht}

Stiftung Haus der kleinen Forscher

1 Die Zieldimensionen für Multiplikatorinnen und Multiplikatoren als Grundlage für die (Weiter-)Entwicklung der Stiftungsangebote

2 Das Qualitätssystem für Fortbildung

3 Verstetigung des Qualitätssystems und wissenschaftliche Begleitung

4 Ausblick 


\section{Die Zieldimensionen für Multiplikatorinnen und Multiplikatoren als Grundlage für die (Weiter-)Entwicklung der Stiftungsangebote}

Fachlich fundierte Zieldimensionen dienen der Stiftung als Orientierungsgrundlage für ihre inhaltlichen Angebote und spezifizieren, welche Ziele mit bestimmten Angeboten erreicht werden sollen. Darüber hinaus bilden die Zieldimensionen eine wichtige Grundlage für die wissenschaftliche Begleitforschung und Evaluation der Stiftungsarbeit. Bisher wurden Zieldimensionen früher naturwissenschaftlicher, technischer, mathematischer sowie informatischer Bildung für Kinder und pädagogische Fach- und Lehrkräfte erarbeitet und publiziert (Stiftung Haus der kleinen Forscher, 2013, 2015b, 2017c, 2018b). In 2019 erscheinen die Zieldimensionen einer Bildung für nachhaltige Entwicklung für Kinder und pädagogische Fach-, Lehr- und Leitungskräfte (Stiftung Haus der kleinen Forscher, in Vorbereitung).

Der vorliegende Band beinhaltet Zieldimensionen für die Multiplikatorinnen und Multiplikatoren früher MINT-Bildung, auch aus Sicht einer Bildung für nachhaltige Entwicklung, und die daraus resultierenden Empfehlungen für die (Weiter-)Entwicklung der inhaltlichen Stiftungsangebote für die Trainerinnen und Trainer der Initiative.

Die Expertise von Köller et al. beschreibt mit den Zieldimensionen für Multiplikatorinnen und Multiplikatoren früher MINT-Bildung, was professionelle Trainerinnen und Trainer in der frühen MINT-Bildung auszeichnet und damit zum Gelingen von Weiterbildungen beiträgt. Die Autorinnen und Autoren empfehlen insbesondere:

- Motivationale Orientierungen in Bezug auf MINT-Bildung,

- Überzeugungen und Werthaltungen,

- Fachwissen in Mathematik, Informatik, Naturwissenschaft und Technik,

- Fachdidaktisches Wissen in Mathematik, Informatik, Naturwissenschaft und Technik sowie

- Allgemein-pädagogisches Wissen, Beratungs- und Organisationswissen.

Die vorliegende Empfehlung erfolgte vor dem Hintergrund sowohl theoretischer als auch praxisorientierter Überlegungen. Motivationale Orientierungen in Bezug auf MINT-Bildung sind grundlegend für eine nachhaltige Implementierung früher MINT-Bildung in Kitas, Horten und Grundschulen. Denn sind Trainerin- 
nen und Trainer an MINT interessiert, sind eine positive emotionale Haltung zur MINT-Bildung und positive Selbstwirksamkeitserwartungen eher wahrscheinlich. Diese emotionalen und motivationalen Aspekte unterstützen wiederum bei der konkreten Umsetzung mathematischer, informatischer, naturwissenschaftlicher und technischer Bildung. Hierfür sollten Trainerinnen und Trainer ein grundlegendes Verständnis der (prozessbezogenen) Gemeinsamkeiten der MINT-Fachdisziplinen haben und zusätzlich vertieftes Fachwissen sowie fachdidaktisches Wissen in mindestens einer MINT-Fachdisziplin mitbringen. Über allgemeines pädagogisch-psychologisches Wissen und Können sollten sie verfügen, um effektive Lernumgebungen gestalten und Lernprozesse in Fortbildungen begleiten zu können. Denn erst die Begleitung von Lernprozessen führt dazu, dass Merkmale erfolgreicher Fortbildungen auch zum Tragen kommen und Wirkungen auf die professionelle Kompetenz der Fachkräfte, die Implementations- und Prozessqualität in der Bildungseinrichtung und darüber vermittelt auf die Kompetenzentwicklung der Kinder entfalten können.

Die Stiftung folgt diesen Empfehlungen zu den Zieldimensionen für Multiplikatorinnen und Multiplikatoren in der MINT-Bildung. Für die Trainerinnen und Trainer wurden zentrale Zieldimensionen mit relevanten Unterdimensionen zusammengefasst und mit leicht angepassten Bezeichnungen versehen (siehe Abbildung 9). Die empfohlene Zieldimension Motivationale Orientierungen in Bezug auf MINT-Bildung entspricht dem Zielbereich Motivation und Selbstvertrauen in Bezug auf MINT-Bildung. Die Zieldimension Überzeugungen und Werthaltungen geht ein in das Professionelle Rollen- und Selbstverständnis als Lernbegleitung. Die Zieldimension Fachwissen (inhalts- und prozessbezogen) findet sich im MINT Fach- und Vorgehenswissen. Die Zieldimension Fachdidaktisches Wissen entspricht den Pädagogischen Handlungsstrategien in Bezug auf MINT-Bildung und die Zieldimension Allgemein-pädagogisches Wissen und Beratungswissen dem Bereich Erwachsenenbildung (siehe Abbildung 6 und Abbildung 9). 


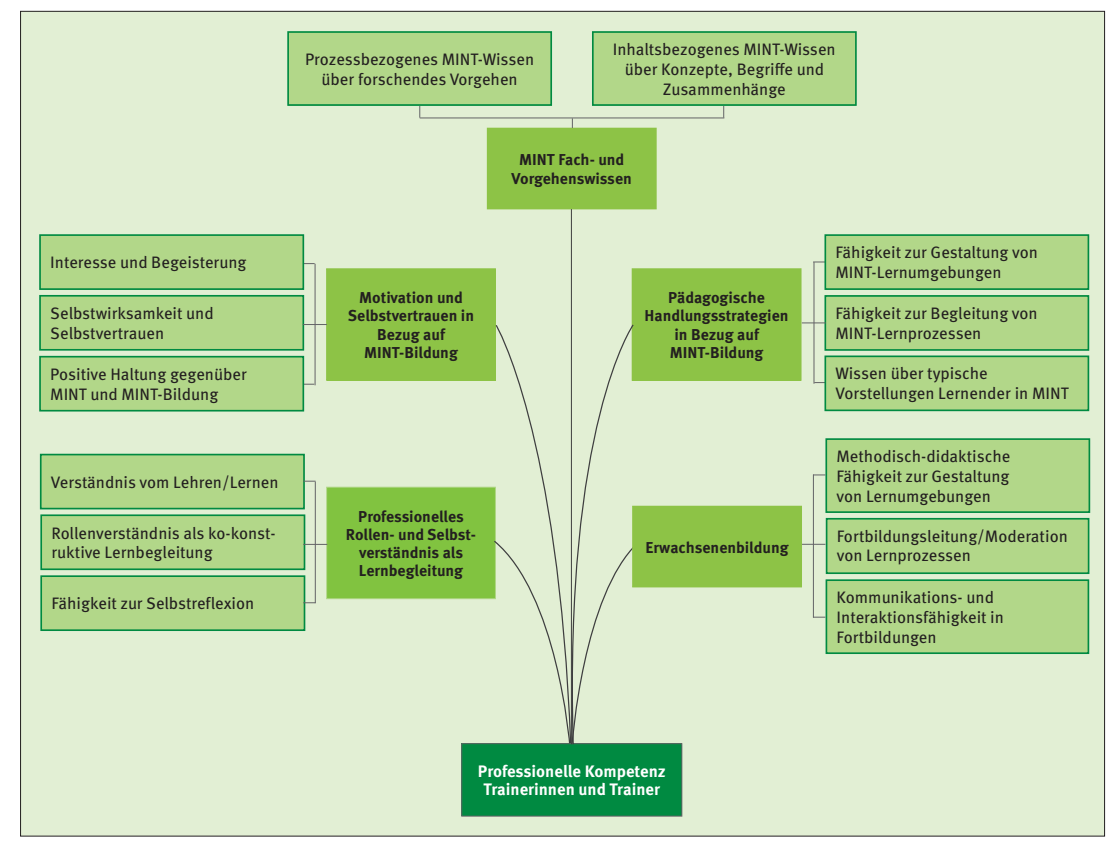

Abbildung 9. Fokussierte Zieldimensionen für Trainerinnen und Trainer des „Hauses der kleinen Forscher" (Stand 2018)

Die hier dargestellten Zieldimensionen für Trainerinnen und Trainer entsprechen dem im Jahr 2018 gewählten Fokus. Es ist gut denkbar, dass sich mit der Weiterentwicklung der Initiative neue Akzentuierungen in Bezug auf bestimmte Zieldimensionen ergeben. So verfolgt die Stiftung derzeit $u$. a. einen stärker systemischen Ansatz, der die Entwicklung gesamter Bildungseinrichtungen unterstützt - etwa in den Angeboten für Kita-Leitungen im Bereich einer Bildung für nachhaltige Entwicklung und in neuen Projekten. Zur Förderung der pädagogischen Qualität früher MINT-Bildung plant die Stiftung „Haus der kleinen Forscher“ ab Frühjahr 2019 zwei Projekte, die die Qualitätsentwicklung in Kitas aus einer systemischen Perspektive (im Sinne eines Whole Institution Approach), auch unter Berücksichtigung der Leitungsebene, in den Blick nimmt (siehe Kapitel 4 Ausblick).

Der Kommentar von Heike Molitor zeigt auf, dass MINT-Bildung und Bildung für nachhaltige Entwicklung in einem konstruktiven Miteinander Bildungs- und Reflexionsprozesse anregen können. Heike Molitor diskutiert die Anforderungen an Multiplikatorinnen und Multiplikatoren der frühen MINT-Bildung aus Sicht einer Bildung für nachhaltige Entwicklung und beschreibt eine Reihe von Überschneidungen zwischen beiden Bildungsbereichen. Ergänzend hebt sie Aspekte hervor, die in Bezug auf die Beförderung einer nachhaltigen Entwicklung in päda- 
gogischen Einrichtungen wie auch in der Gesellschaft von besonderer Bedeutung sind. Multiplikatorinnen und Multiplikatoren einer (MINT-)Bildung für nachhaltige Entwicklung sollten insbesondere Organisationswissen (zur Beförderung eines Whole Institution Approach, Ferreira, Ryan, \& Tilbury, 2006) sowie Motivation, Fachwissen und fachdidaktisches Wissen in Bezug auf nachhaltige Entwicklung mitbringen. Sie sollten reflektierte Werthaltungen zu Fragen der sozialen Gerechtigkeit und des Mensch-Natur-Verhältnisses haben und über Reflexions-, Bewertungs- und Handlungskompetenz in einem Nachhaltigkeitskontext verfügen.

Ähnliche Überlegungen finden sich auch in den Zieldimensionen für Multiplikatorinnen und Multiplikatoren früher MINT-Bildung, etwa zum epistemologischen Wissen (siehe Kapitel 3.3.5 Epistemologisches Wissen im Abschnitt von Köller et al.). So sollten Multiplikatorinnen und Multiplikatoren die historische, kulturelle und gesellschaftliche Bedingtheit der Wissenschaften (Science in Context) kennen und dies an Beispielen wie Umweltschutz erläutern können. Zudem sollten sie um die Bedeutung der MINT-Fächer für die berufliche und gesellschaftliche Teilhabe wissen. Die Auseinandersetzung mit Werten, Fragen der gesellschaftlichen Verantwortung, Technikfolgenabschätzung und Ethikdiskussionen sind wichtiger Bestandteil von Wissenschaft und damit auch von MINT-Bildung. Dabei geht es u. a. darum, Wissen durch geeignete Denk- und Handlungsweisen zu erwerben, zu erweitern, kritisch zu reflektieren und anzuwenden, grundlegende Zusammenhänge selbstständig zu erschließen, zu beurteilen und darauf beruhend Entscheidungen zu treffen (Gesellschaft für Didaktik des Sachunterrichts, 2002, 2013). Eine vertiefende Auseinandersetzung mit einer Bildung für nachhaltige Entwicklung und deren Bezüge zur frühen MINT-Bildung findet sich im 2019 erscheinenden Band 12 der wissenschaftlichen Schriftenreihe der Stiftung (Stiftung Haus der kleinen Forscher, in Vorbereitung).

Die Zieldimensionen für Multiplikatorinnen und Multiplikatoren früher MINT-Bildung und der Kommentar aus Sicht einer Bildung für nachhaltige Entwicklung sind Grundlage für das Qualitätssystem für Fortbildung in der Initiative „Haus der kleinen Forscher“, das im Rahmen des Projekts Trainerakademie 2.0 entwickelt wurde. Wesentliche Elemente des Qualitätssystems sind das Bewerbungs- und Akkreditierungsverfahren, eine am individuellen Bedarf orientierte Qualifizierung und Unterstützung sowie eine regelmäßige Re-Akkreditierung der Trainerinnen und Trainer. Das Qualitätssystem für Fortbildung wurde mit Trainerinnen und Trainern sowie mit Netzwerkpartnern gemeinsam entwickelt. Sie wirkten in Fokusgruppen mit, gaben kritisches Feedback (z. B. in Interviews), diskutierten mit der Stiftung (z. B. bei der Jahrestagung 2017) und erprobten alle neuen Angebote. Im Folgenden wird das Qualitätssystem für Fortbildung ausführlich beschrieben. 


\section{Das Qualitätssystem für Fortbildung}

Das Qualitätssystem für Fortbildung greift die bisherigen Maßnahmen zur Qualitätssicherung und Qualitätsentwicklung der Stiftungsangebote auf (wie z. B. die fachlich fundierte Entwicklung neuer Themen, Materialien und Fortbildungskonzepte, die Berücksichtigung von Rahmenlehrplänen, die Pilotierung von Fortbildungen in der Praxis, die Begleitung der Multiplikatorinnen und Multiplikatoren und die Evaluation der Bildungsangebote), systematisiert und erweitert diese.

Das Qualitätssystem für Fortbildung fokussiert auf die Qualität bei der Durchführung von Fortbildungen. Es beinhaltet die systematische Bewerbung, Akkreditierung und eine regelmäßige Re-Akkreditierung der Multiplikatorinnen und Multiplikatoren, bei der die Trainerinnen und Trainer in Absprache mit dem lokalen Netzwerk eine Vereinbarung mit der Stiftung zu bestimmten Qualitätsanforderungen schließen. Diese Qualitätsanforderungen sind in der Initiative (Website, Online-Plattform für Netzwerkpartner, Multiplikatorinnen und Multiplikatoren, Veröffentlichungen wie diese Schriftenreihe) transparent dargestellt. Sowohl bei der Bewerbung und Akkreditierung als auch bei der Re-Akkreditierung werden drei Ebenen abgebildet: die Passung zum Netzwerk, die Passung zur Stiftung „Haus der kleinen Forscher" und die fachliche Eignung als Trainerin bzw. Trainer (siehe Abbildung 10).

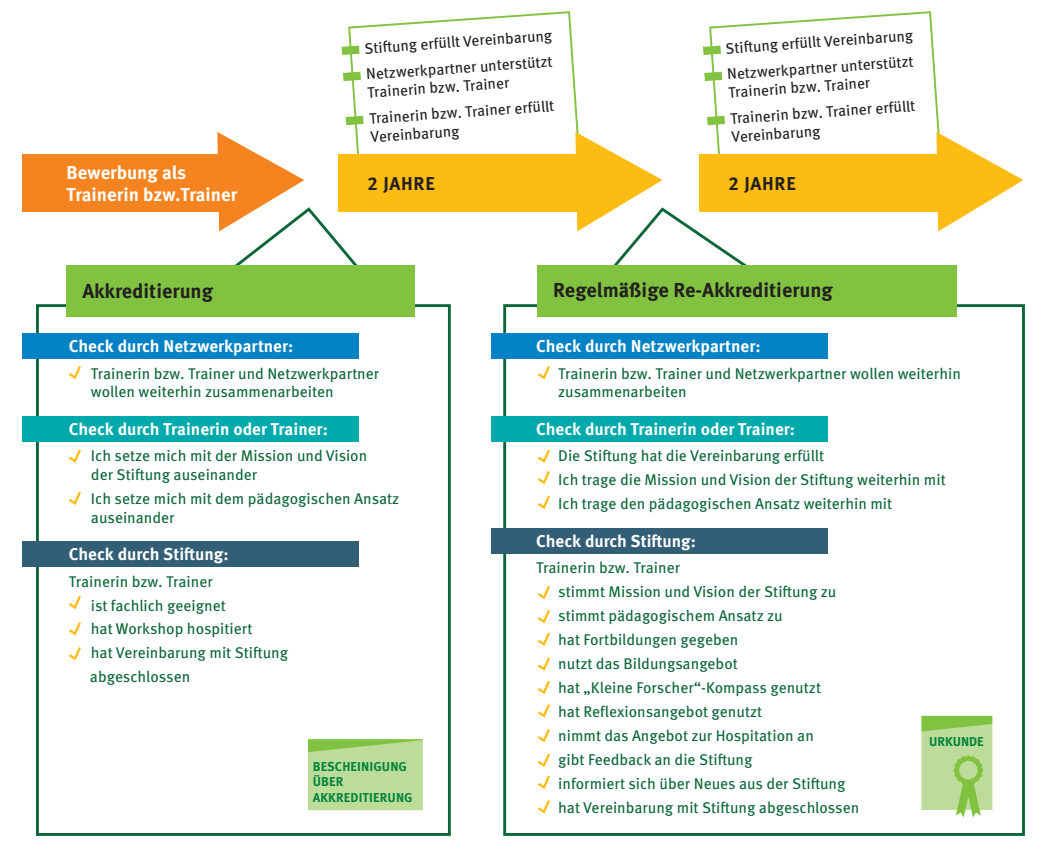

Abbildung 10. Prozess der Bewerbung, Akkreditierung und regelmäßigen Re-Akkreditierung der Trainerinnen und Trainer 
Das Qualitätssystem für Fortbildung systematisiert und erweitert auf Basis der Zieldimensionen die Anforderungen an Trainerinnen und Trainer und macht diese explizit. Die Gegenüberstellung der Sicht von (qualifizierenden) Trägern und Lehrenden (die Fortbildungen durchführen) im Projekt Konturen der Professionalisierung in der beruflichen Weiterbildung (Koproff) zeigt, dass solche transparenten Auswahl- und Qualifizierungsprozesse auf der Grundlage vorab definierter Kompetenzanforderungen in beiderseitigem Interesse sind. Denn Aufgaben und Kompetenzen in der frühpädagogischen Weiterbildung können von Trägern und Lehrenden unterschiedlich gewichtet werden (Buhl, Buschle, Freytag, Hippel \& Iller, 2014; Iller, 2015). Transparente Prozesse und Anforderungen führen nicht dazu, dass gegenseitige Erwartungen automatisch erfültt werden. Aber sie ermöglichen es, gegenseitige Erwartungen wahrzunehmen und vor dem Hintergrund eigener Erfahrungen mit den Vorstellungen von sich selbst und der eigenen beruflichen Rolle und Identität anzugleichen und ggf. in entsprechendes berufliches Handeln umzusetzen (Buschle, 2014). Dieses Handeln entspricht nicht unbedingt den formulierten Erwartungen, möglicherweise muss immer wieder der Konflikt sich widersprechender Erwartungshaltungen gelöst werden (Buschle, 2014).

Mit dem neuen Qualitätssystem möchte die Stiftung einen Rahmen für den regelmäßigen Dialog schaffen, in dem sich Trainerinnen und Trainer, Netzwerkpartner und Stiftung kontinuierlich über gegenseitige Erwartungen und Qualitätsanforderungen austauschen. Die im Qualitätssystem beschriebenen Qualitätsanforderungen leiten sich aus der fachlichen Fundierung zu Zieldimensionen für Weiterbildnerinnen und Weiterbildner in der frühen MINT-Bildung sowie aus den praktischen Empfehlungen und Erfahrungen ab, die die Stiftung im fortwährenden (und im Projekt Trainerakademie noch einmal intensivierten) Austausch mit den Trainerinnen und Trainern und Netzwerkpartnern gesammelt hat.

\subsection{Bewerbung, Akkreditierung und Re-Akkreditierung der Trainerinnen und Trainer}

Bei der Bewerbung werden die Voraussetzungen erfasst, die potenzielle Trainerinnen und Trainer mitbringen, vor allem auch solche Voraussetzungen, die sie bereits mitbringen sollten, weil sie durch die Teilnahme an Fortbildungen nur sehr schwer veränderbar sind. Dazu gehören die Motivation und das Selbstvertrauen, als Trainerin oder Trainer in der MINT-Bildung (für nachhaltige Entwicklung) tätig zu sein, die Motivation, sich als Trainerin oder Trainer weiterzuentwickeln, oder relevante Überzeugungen und Werthaltungen zum Lehren und Lernen (etwa das Mittragen des pädagogischen Ansatzes eines entdeckend-forschenden Lernens). Darüber hinaus werden der berufliche Hintergrund (Ausbildung, Studium, 
besuchte Fort- und Weiterbildungen) sowie die bisherige Berufserfahrung in der MINT-Bildung (für nachhaltige Entwicklung) erfasst, um Rückschlüsse auf das MINT Fach- und Vorgehenswissen, die Erfahrungen in der Erwachsenenbildung und in der Lernbegleitung von Kindern und Erwachsenen im Sinne pädagogischer Handlungsstrategien ziehen zu können. Trainerinnen und Trainer, die sich für eine Tätigkeit im Bereich (MINT-)Bildung für nachhaltige Entwicklung bewerben, werden zusätzlich nach ihrer Motivation für BNE, nach ihren bisherigen Erfahrungen und nach den besuchten wie selbst durchgeführten Fort- und Weiterbildungen zu Nachhaltigkeit oder zu Leitung und Führung im Bildungs- oder Sozialwesen gefragt. Erfüllen die potenziellen Trainerinnen und Trainer die Voraussetzungen an die motivationale wie fachliche Eignung und wollen Netzwerkpartner und Trainerin bzw. Trainer zusammenarbeiten, so werden sie als Fortbildnerinnen und Fortbildner in der Initiative „Haus der kleinen Forscher“ akkreditiert. Bei der Akkreditierung unterzeichnen die Stiftung und die Trainerinnen bzw. Trainer eine Vereinbarung.

Die Vereinbarung orientiert sich an den Zieldimensionen für Trainerinnen und Trainer und beinhaltet Folgendes:

- Trainerinnen und Trainer nutzen das Bildungsangebot bzw. die Qualifizierungsangebote der Stiftung und führen selbst Fortbildungen in der Bildungsinitiative durch.

Denn durch den Besuch und die Durchführung von Fortbildungen erwerben Trainerinnen und Trainer Wissen über Ko-Konstruktion, forschendes Lernen und Erwachsenenbildung. Zudem erwerben sie fachliches und fachdidaktisches Wissen und Erfahrungen für die Fortbildungsdurchführung. Diese Weiterbildungspraxis erweitert wiederum das Wissen über die Planung, Durchführung und Nachbereitung von Fortbildungen und stärkt die professionelle Handlungskompetenz.

- Trainerinnen und Trainer nutzen Reflexionsangebote der Stiftung wie das Reflexions- und Orientierungstool „Kleine Forscher“-Kompass, Videocoaching oder Hospitationen.

Durch die Nutzung dieser Angebote können Trainerinnen und Trainer ihr Wissen und ihr Handeln reflektieren (siehe Kapitel 2.3 Reflexionsangebote für Trainerinnen und Trainer). Hospitationen und Videocoaching ermöglichen es Trainerinnen und Trainern darüber hinaus, das Handeln in konkreten Fortbildungssituationen im persönlichen Austausch mit der Stiftung zu reflektieren und einzuschätzen. Die Stiftung kann auf dieser Grundlage individuell beraten und Rückmeldungen und Empfehlungen geben, mit welchen Qualifizie- 
rungsangeboten sich die Trainerinnen und Trainer in welchen Zieldimensionen weiterentwickeln können. Die Reflexion über die eigene Professionalität ist eine wesentliche Voraussetzung für das professionelle Handeln von Weiterbildnerinnen und Weiterbildnern (Buschle, 2014).

- Trainerinnen und Trainer halten sich über Neuigkeiten aus der Stiftung auf dem Laufenden, z. B. über angepasste Fortbildungskonzepte, und geben der Stiftung Feedback, z. B. über die Teilnahme an Befragungen, an Fokusgruppen oder im persönlichen Austausch. Denn nur so kann die Stiftung ihre Angebote stetig weiterentwickeln und verbessern.

Wichtiges Ziel der vereinbarten Maßnahmen ist es, die professionelle Kompetenz der Trainerinnen und Trainer zu stärken und die Qualität der Fortbildungen in der Initiative für pädagogische Fach- und Lehrkräfte kontinuierlich zu sichern.

Die Re-Akkreditierung erfolgt zwei Jahre nach der Akkreditierung und dann regelmäßig alle zwei Jahre. Bei der Re-Akkreditierung stimmen sich Trainerin bzw. Trainer mit der Stiftung und der Netzwerkkoordination bewusst ab, ob die Zusammenarbeit im gemeinsamen Verständnis weiter fortgesetzt wird. Denn Kompetenzen und Haltungen werden nicht einmalig, sondern über die gesamte Lebensspanne immer weiterentwickelt. An diesem Prozess ist der ganze Mensch mit seinen Motivationen, Überzeugungen, Gefühlen und biografischen Erfahrungen beteiligt (Fröhlich-Gildhoff et al., 2011). Bei der Re-Akkreditierung prüfen Trainerinnen und Trainer, ob sie weiterhin motiviert und gewillt sind, die Vision, die Mission und den pädagogischen Ansatz der Stiftung mitzutragen und sich mit der Initiative weiterzuentwickeln. Dabei wird geschaut, ob die Vereinbarung von Trainerinnen und Trainern und von der Stiftung eingehalten wurde. Denn auch die Stiftung verpflichtet sich mit der Vereinbarung, den Trainerinnen und Trainern entsprechende Angebote zur Verfügung zu stellen. Bei der Re-Akkreditierung erhalten die Trainerinnen und Trainer eine Urkunde, die das Engagement in der Initiative „Haus der kleinen Forscher" anerkennt und bescheinigt.

Transparente Prozesse und Anforderungen wie der Prozess rund um die Bewerbung, Akkreditierung und ReAkkreditierung bieten den Trainerinnen und Trainern die Grundlage, eine informierte Entscheidung für die Zusammenarbeit mit der Stiftung zu treffen, und der Stiftung einen Rahmen für die professionelle, individuelle Qualifizierung und Unterstützung der Trainerinnen und Trainer bei ihrer Tätigkeit als Multiplikatorinnen und Multiplikatoren in der frühen MINT-Bildung. Die individuelle Qualifizierung und Unterstützung der Trainerinnen und Trainer beinhaltet zum einen die weiter- und neuentwickelten Qualifizierungsangebote wie 
Profilfortbildungen und Online-Angebote und zum anderen Reflexionsangebote wie Videocoaching oder das internetbasierte Reflexions- und Orientierungstool „Kleine Forscher“-Kompass.

\subsection{Qualifizierungsangebote für Trainerinnen und Trainer}

Die Qualifizierungsangebote für Trainerinnen und Trainer beinhalteten vor dem Projekt Trainerakademie 2.0 vor allem Präsenzfortbildungen und pädagogische Materialien zu mathematischen, informatischen, naturwissenschaftlichen und technischen Themen sowie einzelne Online-Angebote, z. B. zum forschenden Lernen.

In einem ersten Schritt wurden die zu Projektbeginn bestehenden Qualifizierungsangebote mit den Zieldimensionen für Trainerinnen und Trainer abgeglichen, um festzustellen, für welche Zieldimensionen es bereits ausreichend viele und für welche es bisher zu wenige oder noch keine Angebote gibt.

Es zeigte sich, dass die Zieldimension Motivation und Selbstvertrauen im Bewerbungsverfahren und in allen thematischen Fortbildungen adressiert wird. Denn die Fortbildungen der Stiftung zielen in ihrer Gestaltung stets darauf ab, den pädagogischen Fach- und Lehrkräften wie auch den Trainerinnen und Trainern einen eigenen positiven Zugang zu MINT-Themen zu ermöglichen und eine offene, forschende Haltung zu entwickeln.

Das MINT Fach- und Vorgehenswissen wird ebenfalls in den thematischen Fortbildungen und in den zugehörigen pädagogischen Materialien gefördert. Denn die Fortbildungen zum Entdecken und Forschen orientieren sich am so genannten Forschungskreis (Marquardt-Mau, 2011; Stiftung Haus der kleinen Forscher 2015a) bzw. an Werkzeugen zur Lernbegleitung, die die Methoden der jeweiligen MINT-Disziplinen veranschaulichen (Mathematik-Kreis, Technik-Kreis, Informatik-Kreis, Stiftung Haus der kleinen Forscher, 2019). Diese Kreise beschreiben die prozesshafte, zyklische Vorgehensweise beim wissenschaftlichen Denken und Handeln und regen dazu an, sich durch eigene Aktivität und forschendes Vorgehen mathematische, informatische, naturwissenschaftliche und technische Zusammenhänge zu erschließen. Die pädagogischen Materialien wie etwa die Broschüre zum pädagogischen Ansatz, die Themenbroschüren und die Entdeckungs- und Forschungskarten enthalten ebenfalls Informationen zum entdeckend-forschenden Vorgehen und fachliche Hintergrundinformationen zu den jeweiligen Naturphänomenen sowie zu mathematischen, informatischen oder technischen Fragestellungen. 
Das professionelle Rollen- und Selbstverständnis und die pädagogischen Handlungsstrategien werden ebenfalls in allen thematischen Fortbildungen behandelt. Darüber hinaus bietet die Stiftung mit der so genannten „Forscherzeit“ am Lernort Forscherwelt Blossin ein besonderes Angebot für die Unterstützung von Pädagoginnen und Pädagogen beim forschenden Lernen mit Kindern. Hier stehen die Gestaltung kindgerechter Lernumgebungen, die Begleitung kindlicher Lernprozesse und typische Kindervorstellungen, die direkte Interaktion mit Kindern und eine individuelle Rückmeldung an die Teilnehmenden im Mittelpunkt. Während auch Trainerinnen und Trainer an dieser Forscherzeit teilnehmen können, gab es ein vergleichbares Angebot zur Unterstützung der Trainerinnen und Trainer bei ihrer erwachsenenpädagogischen Arbeit vor der Trainerakademie 2.0 noch nicht. Das heißt, dass besonders in den Bereichen Professionelles Rollenund Selbstverständnis, Pädagogische Handlungsstrategien sowie Erwachsenenbildung Angebote ausgebaut und ergänzt werden sollten.

In einem zweiten Schritt wurden für diese Zieldimensionen neue Qualifizierungsformate entwickelt (für die Zieldimension Professionelles Rollen- und Selbstverständnis wurden zudem weitere Reflexionsangebote entwickelt, siehe Kapitel 2.3.1 Videocoaching). Für die (Weiter-)Qualifizierung in den Bereichen Pädagogische Handlungsstrategien und Erwachsenenbildung wurden neue Präsenzfortbildungen entwickelt, so genannte Profilfortbildungen. Die Profilfortbildungen behandeln Themen wie Moderation, Kommunikation, Gruppendynamik, Visualisierung oder Lernbegleitung und werden seit 2017 zweimal jährlich in Form eines Sommer- bzw. Wintercampus für Multiplikatorinnen und Multiplikatoren angeboten. Beim Sommer- bzw. Wintercampus kommen die Trainerinnen und Trainer nach Berlin, besuchen zwei je zweitägige Profilfortbildungen, hospitieren in einer zertifizierten „Haus der kleinen Forscher“-Kita und nehmen an einem kulturellen Rahmenprogramm teil. Zudem werden die Profilfortbildungen auf Anfrage als netzwerkinterne Fortbildungen vor Ort organisiert, was zugleich den netzwerkinternen Austausch fördert. Am Sommer- bzw. Wintercampus und an weiteren Profilfortbildungen haben bislang 306 Trainerinnen und Trainer teilgenommen, die netzwerkinternen Profilfortbildungen nutzten bisher 45 Trainerinnen und Trainer (Stand 01.09.2018). Besonders nachgefragt sind die Themen Lernbegleitung sowie Moderation und Methoden, die zum Teil auch als Blended-Learning-Angebote existieren.

Zusätzlich zu den Profilfortbildungen und dem bestehenden Online-Campus für Trainerinnen und Trainer wurden verschiedene Online-Angebote entwickelt, die die Fortbildungen inhaltlich vertiefen oder ergänzen, z. B. ein offener Online-Kurs zur naturwissenschaftlichen Erkenntnisgewinnung, ein moderierter Online-Kurs 
zu Gruppendynamik und ein Webinar zur Körpersprache. Wie die Präsenzformate werden alle Online-Kurse fortlaufend evaluiert. Untersucht wird: 1) ob die Teilnehmenden zufrieden mit der zeitlichen Organisation, der Struktur, den Methoden und den Aufgaben sind und 2) ob die zur Verfügung gestellten Online-Tools auch genutzt werden. Insgesamt zeigt sich, dass die Trainerinnen und Trainer mit den Online-Angeboten sehr zufrieden sind und diese häufig auch weiterempfehlen. Auch die zur Verfügung gestellten Online-Tools wie Blogs, Chats oder Foren werden häufig genutzt. Weshalb manche Trainerinnen und Trainer die Online-Tools nicht nutzen (No show) oder sogar den Kurs abbrechen (Drop out) untersuchte das Umfragezentrum Bonn in einer Studie mit 17 Telefoninterviews (Krüger \& Laubach, 2018). Die Interviews zeigten, dass die Online-Angebote zwar eine hohe zeitliche und räumliche Flexibilität bieten, aber aufgrund des generellen Zeitmangels sowie aufgrund technischer Anforderungen und Schwierigkeiten von den Interviewten nur eingeschränkt genutzt werden. Zudem werden die Online-Angebote zum Teil als zu oberflächlich und zu verpflichtend wahrgenommen. Krüger und Laubach (2018) empfehlen daher, die Online-Angebote zu verschiedenen Tageszeiten anzubieten, eine technische Support-Hotline einzurichten und deutlich zu kommunizieren, dass die Online-Angebote das Präsenzangebot ergänzen und nicht ersetzen sollen. Darüber hinaus sollten die Online-Angebote im Rahmen von Präsenzfortbildungen beworben und erläutert werden. Die Stiftung hat diese Erkenntnisse aufgegriffen und entwickelt die Online-Angebote und die Rahmenbedingungen für ihre Nutzung entsprechend weiter.

In einem dritten Schritt wurden zusätzlich zur systematischen Analyse und Weiterentwicklung der Qualifizierungsangebote, basierend auf den Zieldimensionen für Trainerinnen und Trainer, die Beschreibungen aller Präsenz- und Online-Angebote angepasst. Diese werden nun verstärkt kompetenz- bzw. lernzielorientiert formuliert und zeigen auf, welche Zieldimensionen bzw. Kompetenzbereiche bestimmte Angebote adressieren. Denn die mit dem Kompetenzkonzept einhergehende Verschiebung von einer Input- zu einer Outcome-Orientierung führt auch zur Betonung des Könnens bzw. des beschreibbaren Verhaltens, das am Ende einer Qualifizierungsmaßnahme stehen soll (Müller et al., 2016). Zusammen mit den Empfehlungen im „Kleine Forscher“-Kompass sollen die Beschreibungen der Angebote die Orientierung im Bildungsangebot der Stiftung und die Auswahl geeigneter Fortbildungen erleichtern. 


\subsection{Reflexionsangebote für Trainerinnen und Trainer}

\subsubsection{Videocoaching}

Für die Zieldimension Professionelles Rollen- und Selbstverständnis wurden zusätzlich zum Hospitationsprogramm, das die Stiftung seit 2012 anbietet, fünf Reflexionsangebote für Trainerinnen und Trainer entwickelt: Videocoaching, Videocoaching im Team, Reflexionstreffen, Online-Coaching und Formate zur Unterstützung der Selbstreflexion.

Videocoaching ist eine Form der onlinegestützten Reflexion. Dabei zeichnen die Trainerinnen und Trainer Sequenzen einer von ihnen durchgeführten Fortbildung mit der Kamera auf und reflektieren diese anschließend mit einer Referentin oder einem Referenten der Stiftung in einem strukturierten Coaching-Gespräch. Reflexionsangebote wie Videocoachings entsprechen besonders gut der Kompetenz- und Handlungsorientierung der Zieldimensionen. Denn sie ermöglichen es - zusätzlich zur Erarbeitung theoretischen Wissens in den Fortbildungen und zusätzlich zur Erfassung des Wissens im „Kleine Forscher“-Kompass (s. u.) - das eigene praktische Können, begangene Fehler sowie Erfolge zu analysieren und auf dieser Basis künftige Arbeitsprozesse zu verändern. Müller et al. (2016) nennen dies die Zirkularität von Kognition und Handeln, die eine spezifische Anforderung für die methodisch-didaktische Gestaltung von Fort- und Weiterbildungen ist (siehe auch Fröhlich-Gildhoff et al., 2011). Die Angebote zur gemeinsamen Praxisreflexion wie das Videocoaching im Team berücksichtigen darüber hinaus, dass Professionalität nicht nur ein individuelles Vermögen ist, sondern immer auch durch die Situation, Dinge, Räume, weitere Personen und vieles mehr bestimmt ist (Starke, 2017).

Die Entwicklung des Videocoachings wurde von externen Expertinnen und Experten begutachtet und wissenschaftlich begleitet und evaluiert. Stefan Brée, Professor für Bildung in der Kindheit an der HAWK Hildesheim, hat das Videocoaching der Stiftung in der Forscherwelt Blossin selbst erlebt und daraufhin sein Forschungsprojekt „Forscherzeiten im Familienzentrum Rethen“ daran orientiert. Markus Bach, Leiter des Marte Meo Instituts, begrüßt die wertschätzende Kommunikation der Referenteninnen und Referenten beim Videocoaching und hebt deren ausgeprägte fachliche sowie zwischenmenschliche Professionalität hervor. Claudia M. König, Kommunikations- und Erziehungswissenschaftlerin und Videocoachin, zollt den begleitenden Materialien Anerkennung und ist überzeugt, dass die Trainerinnen und Trainer durch das Videocoaching ihr Selbstbild erweitern und sich selbst besser kennenlernen können. 
In einem Lehrforschungsprojekt an der FU Berlin wurde untersucht, wie Trainerinnen und Trainer das Videocoaching erleben - vor allem im Unterschied zur Hospitation einer Fortbildung vor Ort - und welche Effekte es auf ihre Haltung und ihre Selbstreflexion hat. Die Interviews zeigen, dass die Trainerinnen und Trainer die Feedbackgespräche beim Videocoaching und bei der Hospitation als gleichermaßen wertschätzend und hilfreich empfinden. Beim Videocoaching kommt hinzu, dass die Trainerinnen und Trainer gezielt an kleineren, selbst ausgewählten Fragestellungen arbeiten können, z. B. indem sie gezielt bestimmte Filmsequenzen auswählen und andere weglassen. Eine interviewte Trainerin sagte dazu: „Also bei dem Videofeedback wie gesagt war es ja so, ich hab / da hab ich auch / das ist ja kürzer und ich kann es selber auch steuern, was ausgewertet wird. Da hab ich ja einen Einfluss. "Negativ angemerkt werden vor allem organisatorische und technische Schwierigkeiten, so z. B. das fehlende persönliche Treffen oder Probleme bei der Bedienung der Kamera. In zwei Masterarbeiten in Kooperation mit der FU Berlin wurde untersucht, wie das Feedback bei Hospitationen und Videocoachings gestaltet werden sollte, damit es in der Praxis umgesetzt wird, und welche förderlichen bzw. hemmenden Faktoren die Teilnahme an Videocoachings beeinflussen. Es zeigte sich, dass Trainerinnen und Trainer eher an Videocoachings teilnehmen, wenn sie bereits an einer persönlichen Hospitation teilgenommen haben und wenn sie einen konkreten Bedarf haben, sich einschätzen und beraten zu lassen.

\subsection{2 „Kleine Forscher“-Kompass für Trainerinnen und Trainer}

Der „Kleine Forscher“-Kompass hilft Trainerinnen und Trainer, ihren Wissenstand zu einzelnen Bereichen selbst einzuschätzen und gibt Orientierung zu Angebotsempfehlungen zur Weiterentwicklung. Der „Kleine Forscher“-Kompass ist ein internetbasiertes Reflexions- und Orientierungstool für Trainerinnen und Trainer und läuft in drei Schritten ab. Zuerst beantworten die Trainerinnen und Trainer einige Fragen zu ihrem Wissen über zentrale theoretische Konzepte wie Ko-Konstruktion oder forschendes Lernen und über das Handeln in praktischen Situationen. Denn dieses Wissen kann das tatsächliche Handeln beeinflussen. Daraufhin erhalten die Trainerinnen und Trainer ihre Ergebnisse mit genauen Informationen über die richtigen Antworten und weiterführende Informationen, z. B. zu theoretischen Grundlagen. Zuletzt erhalten die Trainerinnen und Trainer individuelle Empfehlungen, welche Angebote des „Hauses der kleinen Forscher“ sie am besten dabei unterstützen, sich gezielt weiterzubilden. Die Empfehlungen beinhalten eine Information darüber, inwieweit die Angebote zu den Ergebnissen passen und weshalb sich die Angebote lohnen könnten. Die Entwicklung des „Kleine Forscher"-Kompasses erfolgte in mehreren, aufeinander aufbauenden Schritten, die 
hier exemplarisch für die Entwicklung neuer Angebote in der Stiftung etwas ausführlicher erläutert werden.

\section{Entwicklung der Fragen}

Im ersten Schritt wurden auf Grundlage der Zieldimensionen für Trainerinnen und Trainer verschiedene Wissensfragen entwickelt und Selbsteinschätzungsfragen recherchiert (siehe Abbildung 11). Diese beziehen sich nicht auf alle, sondern nur auf einige ausgewählte Dimensionen, die für die Fortbildungstätigkeit beim „Haus der kleinen Forscher" besonders relevant sind und die mittels Online-Fragebogen erfasst werden können. Die Zieldimensionen (siehe Abbildung 9) beinhalten jeweils mehrere Unterdimensionen: Erwachsenenbildung beinhaltet beispielsweise Didaktik und Methoden, Differenzierung, Integration und Förderung, Bildungsforschung, Leistungs- und Lernmotivation, Beratungswissen und Kommunikation etc. Von diesen Dimensionen wurden Didaktik und Methoden, Differenzierung, Integration und Förderung sowie Beratungswissen und Kommunikation genauer operationalisiert und in konkrete Wissens- und Selbsteinschätzungsfragen übersetzt. Im „Kleine Forscher“-Kompass sind insgesamt 82 Fragen zu fünf Zieldimensionen enthalten. Beispielhaft ist das in Abbildung 11 veranschaulicht.

Die Zieldimension Motivation und Selbstvertrauen beinhaltet 27 Selbsteinschätzungsfragen zur Selbstwirksamkeit als Trainerin bzw. Trainer beim „Haus der kleinen Forscher“, zum Interesse an MINT, zum Interesse an der Gestaltung von MINT-Lernumgebungen, zu den Fähigkeiten in MINT und zu den Fähigkeiten der Gestaltung von MINT-Lernumgebungen.

Die Zieldimension Professionelles Rollen- und Selbstverständnis wurde mit acht Wissensfragen zu Ko-Konstruktion und ko-konstruktiver Lernbegleitung operationalisiert. Denn erstens entspricht eine konstruktivistische Didaktik, die selbstorganisiertes und selbstgesteuertes Denken und Handeln fördert, besonders gut der Handlungsorientierung im Kompetenzdiskurs (Fröhlich-Gildhoff et al., 2011) und zweitens ist Ko-Konstruktion der zentrale pädagogische Ansatz der Stiftung.

Die Zieldimension Fach- und Vorgehenswissen wurde mit drei Wissensfragen zu Naturwissenschaften aus der Studie EASI Science erfasst (Early Steps into Science, Steffensky, Anders, Barenthien, Hardy, Leuchter, Oppermann, Taskinen, \& Ziegler, 2018).

Die Zieldimension Pädagogische Handlungsstrategien in der MINT-Bildung bezieht sich auf das forschende Lernen (Mathematik-, Informatik-, Forschungsund Technikkreis; Marquardt-Mau, 2011; Stiftung Haus der kleinen Forscher 2015a, 2019). Mit acht Wissensfragen wird das Wissen über Schritte und über Handlungsstrategien zur Begleitung forschenden Lernens erfasst. 


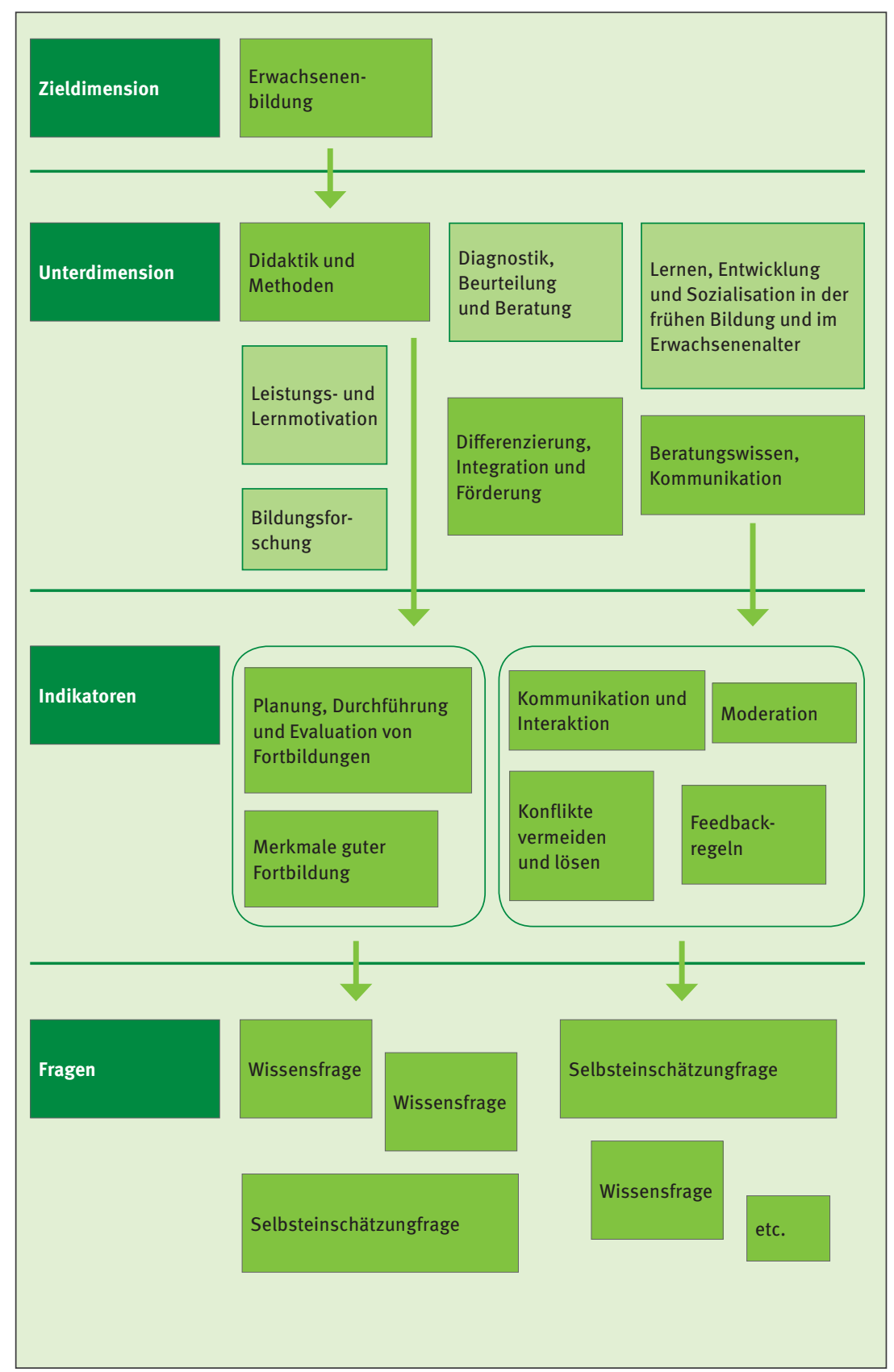

Abbildung 11. Von den Zieldimensionen zu den Fragen im „Kleine Forscher“-Kompass am Beispiel der Dimension Erwachsenenbildung 
Die Zieldimension Erwachsenenbildung wurde mit 16 Wissensfragen zu Didaktik und Methoden und Kommunikation - z. B. Fortbildungen planen, Feedback geben oder Konflikte bearbeiten - und mit 20 Selbsteinschätzungsfragen zur adaptiven Lehrkompetenz und zur Nachbereitung von Fortbildungen erfasst.

Mit dieser Auswahl an Wissensbereichen ist der „Kleine Forscher“-Kompass nicht nur an die Zieldimensionen für Trainerinnen und Trainer anschlussfähig, sondern auch an weitere Konzeptualisierungen des pädagogischen Wissens (für einen Überblick siehe Wißhak, 2017) und an empirische Befunde der Trainingsund Weiterbildungsforschung (Fröhlich-Gildhoff et al., 2011; Iller, 2015; Wißhak, 2017).

Die Wissensfragen haben immer mehrere Antwortmöglichkeiten, von denen jeweils eine als richtig gilt (Single Choice). Die richtigen (wie auch die nicht richtigen) Antworten basieren auf Theorien und Erkenntnissen aus der empirischen Bildungsforschung, den MINT-Fachdidaktiken sowie aus der Erwachsenenbildung und auf den praktischen Erfahrungen der Trainerinnen und Trainer. Die Wissensfragen sind unterschiedlich schwierig, damit sich sowohl Personen mit wenig als auch Personen mit mittlerem oder viel Wissen gut einschätzen können.

Die Selbsteinschätzungsfragen basieren auf validierten Selbsteinschätzungsskalen, z. B. der Skala zur allgemeinen Selbstwirksamkeitserwartung (Jerusalem \& Schwarzer, 1999). Bei den Selbsteinschätzungsfragen geht es um persönliche Auffassungen, Meinungen und Gewohnheiten.

\section{Erste Erprobung der Fragen}

Im zweiten Schritt wurden die Fragen und auch die Ergebnisrückmeldungen und Empfehlungen mehrfach getestet und weiterentwickelt. In einem ersten, stiftungsinternen Pretest wurden Rückmeldungen von 94 Mitarbeiterinnen und Mitarbeitern der Stiftung eingeholt, z. B. aus den Bereichen Inhalte und Fortbildungen oder Administration und Infrastruktur. Diese Rückmeldungen führten zur grundlegenden Überarbeitung der Wissensfragen: die Mehrzahl der bestehenden Wissensfragen wurde umformuliert, zum Teil wurden auch neue Wissensfragen formuliert. Zudem wurden aus jedem Themenbereich zwei Wissensfragen gestrichen, sodass jedes Thema nun mit sechs anstatt mit acht Wissensfragen erfasst wird. Bei den Selbsteinschätzungsfragen wurden aus Platzgründen ebenfalls elf Fragen zum Interesse und zu den Fähigkeiten in MINT gestrichen. In einem zweiten Pretest wurden die überarbeiteten Fragen von den Mitarbeiterinnen und Mitarbeitern des Bereichs Inhalte und Fortbildungen kommentiert. Daraufhin wurden einige der Fragen noch einmal umformuliert, Antwortoptionen überarbeitet etc. 


\section{Erprobung der Pilotversion}

Im dritten Schritt wurden die Trainerinnen und Trainer und das inhaltlich arbeitende Stiftungsteam zur Erprobung der so genannten Pilotversion des „Kleine Forscher"-Kompasses eingeladen. Die überarbeiteten Fragen, Ergebnisse und Empfehlungen wurden an 618 Trainerinnen und Trainer und an die Mitarbeiterinnen und Mitarbeiter des Bereichs Inhalte und Fortbildungen geschickt. Von den 618 Trainerinnen und Trainern haben in der Zeit vom 20. September bis zum 31. Oktober 2017241 den Link zum Tool geöffnet (39 Prozent), 198 mindestens eine Frage beantwortet (32 Prozent) und 183 alle Fragen beantwortet und die Ergebnisse und Empfehlungen angesehen (30 Prozent). Das dauerte insgesamt durchschnittlich 40 bis 45 Minuten. 173 Trainerinnen und Trainer haben zusätzlich die Befragung zu ihrem Eindruck vom „Kleine Forscher“-Kompass ausgefüllt (28 Prozent). Von den Mitarbeiterinnen und Mitarbeitern haben 31 den „Kleine Forscher"-Kompass genutzt. Im Anschluss wurden Itemstatistiken (z. B. Häufigkeiten, Schwierigkeiten), Nutzungsdaten (z. B. Abbruchstatistiken) und offene Kommentare zur Einstiegsseite, zu den Fragen, Ergebnissen und Empfehlungen ausgewertet.

Zusätzlich wurden die Trainerinnen und Trainer auf der Jahrestagung 2017 um Rückmeldung gebeten. Die Stiftung machte alle bisherigen Entwicklungen transparent und hörte aufmerksam im direkten Kontakt zu, welches Feedback die Trainerinnen und Trainer zu den Fragen und Empfehlungsansichten der Pilotversion gaben. Zudem wurden im Herbst 2017 mit zehn ausgewählten Trainerinnen und Trainern mehrstündige qualitative Nutzungstests und problemzentrierte Interviews zur Akzeptanz und zum subjektiven Nutzen des „Kleine Forscher“-Kompasses durchgeführt. Diese wurden in Kooperation mit der TU Berlin und IRIS e. V. inhaltsanalytisch und themenzentriert-komparativ ausgewertet (Wedel \& Fritz, 2018). Eine Übersicht über die erfolgten Datenerhebungen und Auswertungen findet sich in Tabelle 1. 
Tabelle 1. Datenerhebungen und Auswertungen der Pilotversion des „Kleine Forscher“Kompasses

\begin{tabular}{llll}
\hline Auswertungen & Quantitative & Quantitative & Qualitative \\
& Befragung TuT, & Befragung & Nutzungstests \\
& $N=183$ & MA, $\boldsymbol{N}=31$ & TuT, $\boldsymbol{N}=10$ \\
\hline
\end{tabular}

\title{
Itemanalysen
}

Häufigkeiten, Schwierigkeiten, deskriptive Statistiken, Trennschärfe,

$\mathrm{X}$

Summen- und Mittelwerte

\begin{abstract}
Plausibilitätsprüfung
Korrelation der einzelnen Wissensbereiche und des Gesamtergebnisses mit den Selbsteinschätzungen, mit der Anzahl besuchter und gegebener Fortbildungen, Unterschiede erfahrene und weniger erfahrene

Trainerinnen und Trainer
\end{abstract}

\section{Nutzungsdaten}

Klickstatistiken, Teilnahmezahlen, Abbruch, Filterführung etc.

\begin{tabular}{|c|c|c|c|}
\hline $\begin{array}{l}\text { Offene Kommentare zum Kompass } \\
\text { Inhaltsanalytische Auswertung der } \\
\text { Kommentare zur Einstiegsseite, zu } \\
\text { den Fragen, den Ergebnissen und } \\
\text { den Empfehlungen im Kompass }\end{array}$ & $X$ & $X$ & $X$ \\
\hline $\begin{array}{l}\text { Interviews zu Akzeptanz und } \\
\text { subjektivem Nutzen } \\
\text { Themenzentriert-komparative und } \\
\text { narrationsanalytische Auswertung }\end{array}$ & & & $x$ \\
\hline
\end{tabular}

Anmerkung. TuT $=$ Trainerinnen und Trainer, $\mathrm{MA}=$ Mitarbeiterinnen und Mitarbeiter der Stiftung.

Die Itemanalysen zeigten, dass die Trainerinnen und Trainer, die die Pilotversion erprobten, durchschnittlich 15 von 30 möglichen Punkten erreichen $(M=15.24$, $S D=2.64$ Punkte, Range $=9-21$ Punkte). Besonders viele Punkte erreichten sie in den Bereichen Kommunikation, Ko-Konstruktion sowie MINT Fach- und Vorgehenswissen, etwas weniger in den Bereichen forschendes Lernen und Erwachsenenbildung. Die einzelnen Fragen waren unterschiedlich schwierig, zum Teil auch zu schwer oder zu leicht. Die Plausibilitätsprüfungen zeigten, dass das Ergebnis des Wissenstests positiv mit der Anzahl gegebener und besuchter Fortbildungen zusammenhängt, aber nicht mit den Selbsteinschätzungen zu Interesse, Fähigkeiten und adaptiver Lehrkompetenz. Dabei ist das Ergebnis des Wissenstests bei besonders erfahrenen Trainerinnen und Trainern besser als bei den anderen Trainerinnen und Trainern, und zwar unabhängig vom beruflichen Hintergrund.

Die offenen Kommentare in der quantitativen Befragung der Trainerinnen und Trainer zeigten $u$. a., dass die Wissensfragen zur Reflexion und zum Lernen an- 
regen. Zudem wurden die Wissensfragen als praxisbezogen und abwechslungsreich, die Ergebnisse und Empfehlungen als hilfreich empfunden. Schwierig waren das Antwortformat und der sprachliche Anspruch der Wissensfragen. Darüber hinaus wünschten sich die Trainerinnen und Trainer detailliertere Informationen zu ihren Ergebnissen und noch passendere Empfehlungen.

Die offenen Kommentare in den qualitativen Nutzungstests bezogen sich vor allem auf einzelne Begriffe oder Formulierungen (z. B. auf der Einleitungsseite des Tools), auf Frageinhalte, die Darstellung der Ergebnisse und Angebote sowie auf Layout- und Designaspekte. Die zehn Trainerinnen und Trainer wünschten sich vor allem mehr Informationen zur Einbettung des „Kleine Forscher“-Kompasses in das Qualitätssystem für Fortbildung und detailliertere Rückmeldungen zu ihren Ergebnissen. Insgesamt empfanden die Trainerinnen und Trainer den „Kleine Forscher"-Kompass als eher interessant und hilfreich, als mittelmäßig verständlich und als weder zu kurz noch zu lang. Auch das Prinzip des „Kleine Forscher“-Kompasses mit Einstieg, Fragen, Ergebnissen und Empfehlungen wurde gut angenommen.

Die Interviews zu Akzeptanz und subjektivem Nutzen ergaben u. a., dass die Trainerinnen und Trainer dem „Kleine Forscher“-Kompass eher eine Steuerungsund Selektionsfunktion als eine Orientierungs- und Lernfunktion zuschreiben (Wedel \& Fritz, 2018). Die Trainerinnen und Trainer empfanden die in den Fragen formulierten, theoretisch basierten Handlungsoptionen als zu vage formuliert und implizit normativ. Der Transfer dieser theoretischen Inhalte in die eigene Alltagspraxis schien den interviewten Trainerinnen und Trainern schwerzufallen. Diese wünschten sich daher eine direkte und nachvollziehbare Ergebnisrückmeldung, die den aktuellen Wissenstand und ,Wissenslücken“ nachvollziehbar macht, um eine noch realistischere Selbstwahrnehmung zu unterstützen. Zudem wünschten sich die Trainerinnen und Trainer neben den Informationen zu passenden Weiterbildungen auch niedrigschwellige Verknüpfungen mit anderen selbstgesteuerten Lerngelegenheiten, z. B. Weblinks oder Literatur. Die Stiftung griff die Ergebnisse der Erprobung der Pilotversion auf und entwickelte den „Kleine Forscher“-Kompass wie auch die Erläuterung seiner Orientierungs- und Lernfunktion in der Folge weiter. Dabei wurden auch weitere Einschätzungen unabhängiger Expertinnen und Experten einbezogen.

\section{Reflexion mit unabhängigen Expertinnen und Experten}

Im vierten Schritt wurden die Ergebnisse der Pilotphase des „Kleine Forscher“-Kompasses gemeinsam mit der „Arbeitsgruppe Orientierungsdimensionen“ diskutiert. Die „Arbeitsgruppe Orientierungsdimensionen“ besteht aus externen Expertinnen und Experten der frühkindlichen Bildung, Bildungsforschung, Kompetenzmessung, pädagogischen Diagnostik und Professionalisierung frühpä- 
dagogischer Fach- und Lehrkräfte, die die Stiftung zur Konzeption und zum Aufbau des „Kleine Forscher“-Kompasses berät und fachliche wie praktische Empfehlungen für die Weiterentwicklung des „Kleine Forscher“-Kompasses gibt. Die Arbeitsgruppe besteht aus folgenden Expertinnen und Experten: Prof. Dr. Stefan Brée (HAWK Hildesheim), Prof. Dr. Carola Iller (Universität Hildesheim), Prof. Dr. Olaf Köller (IPN, Kiel), Dr. Bettina Schmidt (Kleine Forscher Hamburg), Lara Weiser (Universität Bonn) und Prof. Dr. Matthias Ziegler (Humboldt Universität zu Berlin).

\section{Entwicklung des „Kleine Forscher“-Kompasses als Teil des Qualitätssystems}

Im fünften Schritt wurde auf Grundlage der Ergebnisse der Pilotphase und des Arbeitsgruppentreffens der „Kleine Forscher“-Kompass konzeptionell, inhaltlich und technisch weiterentwickelt. Ziele der Weiterentwicklung waren vor allem, die Nutzungsfreundlichkeit (Usability) des Tools zu steigern sowie präzisere Empfehlungen zu geben, z. B. durch eine Navigationsleiste, einfachere Filtermöglichkeiten, Informationen über richtig oder falsch beantwortete Fragen, passendere Empfehlungen auf der Basis einzelner Themen statt auf der Basis ganzer Wissensbereiche. Insgesamt sollte der „Kleine Forscher“-Kompass in sich möglichst gut nutzbar und verständlich sein und seine Funktion als Reflexions- und Orientierungsinstrument im Qualitätssystem für Fortbildung gut erfüllen können.

Die Einstiegsseite wurde inhaltlich überarbeitet und durch Antworten auf häufige Fragen (FAQ, englisch Frequently Asked Questions) zum „Kleine Forscher"-Kompass ergänzt. Die Fragen zu den Zieldimensionen wurden angepasst. Die Fragen zu Motivation und Selbstvertrauen gehen nun im neu gestalteten Bewerbungsverfahren auf, die Fragen zum MINT Fach- und Vorgehenswissen im Bereich Fachdidaktisches Wissen über pädagogische Handlungsstrategien. Die Selbsteinschätzungen zu adaptiver Lehrkompetenz und Nachbereitung von Fortbildungen wurden aufgrund des fehlenden Zusammenhangs mit den objektiven Ergebnissen des Wissenstests und aufgrund des geringen Beitrags zu den konkreten Angebotsempfehlungen gestrichen. Dafür wurde das in den Zieldimensionen für Multiplikatorinnen und Multiplikatoren priorisierte, aber bislang nicht abgefragte Thema Differenzierung, Integration und Förderung mit ins Tool aufgenommen. Die restlichen Fragen wurden sprachlich und $z$. T. auch inhaltlich überarbeitet.

Die Ergebnisse werden nun detaillierter und gestuft zurückgemeldet. Auf der ersten Stufe der Ergebnisrückmeldung sehen die Trainerinnen und Trainer, wie viele Fragen sie in den Bereichen Ko-Konstruktion, Forschendes Lernen und Erwachsenenbildung richtig beantwortet haben. Auf der zweiten Stufe sehen sie optional, welche Fragen zu welchem Bereich gehören, welche Antworten sie angekreuzt haben und welches die richtigen Antworten waren. Auf der dritten Stufe erhalten sie optional eine Erläuterung zum theoretischen Hintergrund der Fragen, der richtigen und der falschen Antworten. 
Die Empfehlungen basieren auf einem Abgleich des individuellen Wissens in den Themenbereichen mit den Zielen und Inhalten der Qualifizierungsangebote des „Hauses der kleinen Forscher“. Empfohlen werden spezifische Profilfortbildungen, Online-Kurse, Webinare, Printmaterialien usw. Thematische Fortbildungen wie „Zahlen, Zählen, Rechnen“ oder „Forschen mit Luft“ werden nicht individuell empfohlen, da diese vorrangig in Abstimmung mit dem Netzwerk besucht werden. Die Empfehlungen machen nun explizite Angaben zum Lernformat (z. B. Präsenz- oder Online-Format), zu den adressierten Zieldimensionen und dazu, ob ein Angebot bereits besucht wurde und warum es sich lohnen könnte. Die Beschreibungen beziehen sich zudem immer genau auf die Zieldimensionen, bei denen die jeweilige Person Verbesserungspotenzial hat. Darüber hinaus erleichtert eine Filterfunktion die Übersicht der Empfehlungen.

Seit der Einführung des Qualitätssystems für Fortbildung im September 2018 ist die Nutzung des „Kleine Forscher“-Kompasses fester Bestandteil der Akkreditierung bzw. der Re-Akkreditierung für Trainerinnen und Trainer. Der „Kleine Forscher"-Kompass gibt den Trainerinnen und Trainern eine individuelle Rückmeldung und Empfehlungen, welche Angebote der Stiftung sie am besten dabei unterstützen, sich gezielt weiterzubilden. Zudem dienen die Erkenntnisse aus häufigen Antworten und Empfehlungen als eine Basis für die (Weiter-)Entwicklung zunehmend passgenauer Angebote für Trainerinnen und Trainer.

Der „Kleine Forscher“-Kompass wie auch die Reflexionsangebote Hospitation und Videocoaching sollen die Reflexion der Arbeit der Trainerinnen und Trainer unterstützen und ihnen wie auch der Stiftung Orientierung geben, welche Qualifizierungs- und Unterstützungsangebote die Multiplikatorinnen und Multiplikatoren der Initiative brauchen, um bestmöglich gerüstet Fortbildungen für pädagogische Fach- und Lehrkräfte zu geben. 


\section{Verstetigung des Qualitätssystems und wissenschaftliche Begleitung}

Die Zieldimensionen für Multiplikatorinnen und Multiplikatoren sind Grundlage für das Qualitätssystem für Fortbildung mit optimierter Bewerbung, Akkreditierung, Re-Akkreditierung, Qualifizierung und Unterstützung der Trainerinnen und Trainer. Das Qualitätssystem gilt seit dem 1. September 2018 verbindlich für alle bestehenden sowie für alle neuen Trainerinnen und Trainer in der Initiative „Haus der kleinen Forscher“. Die im Projekt Trainerakademie 2.0 entwickelten Qualifizierungs- und Reflexionsangebote für Trainerinnen und Trainer werden verstetigt und in das reguläre Bildungsangebot der Stiftung überführt. Darüber hinaus werden auf Grundlage der Zieldimensionen sowie auf Grundlage des „Kleine Forscher"-Kompasses neue, bedarfsgerechte Qualifizierungsangebote für Trainerinnen und Trainer entwickelt. Im Zuge der digitalen Weiterentwicklung der Stiftung sollen vor allem die Blended-Learning- und die Online-Angebote ausgebaut werden. Hierfür und auch für alle anderen Qualifizierungsangebote ist es den Autorinnen und Autoren der Expertise zufolge „dringend geboten, [...] die Qualität der Fortbildungen und deren Wirkungen, z. B. auf Überzeugungen sowie das bereichsspezifische Interesse und professionelles Wissen, zu überprüfen“ (siehe Kapitel 5 Fazit und Empfehlungen im Abschnitt von Köller et al.).

Entsprechend wird das Qualitätssystem bei der Einführung und auch in der Zukunft wissenschaftlich begleitet und regelmäßig überprüft. Dazu gehört ein kontinuierlicher Austausch mit Trainerinnen und Trainern (z. B. bei Befragungen, bei der Jahrestagung und weiteren Feedbackmöglichkeiten) und mit Expertinnen und Experten (z. B. im Wissenschaftlichen Beirat der Stiftung und in der Arbeitsgruppe Orientierungsdimensionen). Zudem wird im Winter 2018/19 eine Akzeptanzstudie vom unabhängigen Institut uzbonn durchgeführt, bei der alle Trainerinnen und Trainer sowie Netzwerkkoordinatorinnen und Netzwerkkoordinatoren der Initiative zu ihrer Einschätzung und Bewertung des Bewerbungs-, Akkreditierungs- und Re-Akkreditierungsprozesses befragt werden. Die Ergebnisse der Untersuchung wird die Stiftung nutzen, um die Einführungsphase des Qualitätssystems fundiert zu begleiten. Darüber hinaus ist auch das Qualitätsmanagement weiterhin ein zentrales Element der fortlaufenden Optimierung der Stiftungsangebote für Trainerinnen und Trainer. Das Qualitätsmanagement beinhaltet die regelmäßige und systematische Beschaffung und Analyse von Informationen über die Bildungsangebote und die Bildungspraxis des „Hauses der kleinen Forscher“ sowie die Beratung bei der Entwicklung von Angeboten. 
Mit bedarfsorientierten Befragungen der Trainerinnen und Trainer und mithilfe von Feedback zu Fortbildungen und Materialien wird u. a. geprüft, inwieweit die Angebote des „Hauses der kleinen Forscher“ grundsätzlich geeignet sind, um die beabsichtigten Wirkungen zu erzielen. Die beabsichtigten Wirkungen und die Prozesse auf dem Weg von der Teilnahme an einer Fortbildung bis zu den Kompetenzen sind in der so genannten Wirkungskette definiert. Bei der Erstellung bzw. Überarbeitung der Wirkungskette spielen die Zieldimensionen für Multiplikatorinnen und Multiplikatoren eine zentrale Rolle. Die Zieldimensionen beschreiben die Wirkungsziele, die durch die Fortbildungen auf Ebene der Trainerinnen und Trainer erreicht werden sollen, und leiten die Entwicklung eines neuen Feedbacksystems für Fortbildungen wie auch die Ausrichtung von Projekt- oder Angebotsevaluationen

Bei der Entwicklung von Bildungsangeboten wird sichergestellt, dass neue Qualifizierungs- und Reflexionsangebote auf die Zieldimensionen abgestimmt sind, d. h. dass neue Qualifizierungsangebote die Zielkompetenzen für Trainerinnen und Trainer explizit berücksichtigen. So wird beispielsweise bei der Überarbeitung des Hospitationsprozesses verstärkt auf Aspekte der Fachdidaktik und Erwachsenenbildung geachtet. Begleitend zur inhaltlichen Entwicklung der Prozesse und Qualifizierungsangebote für Trainerinnen und Trainer wird die Stiftung weiterhin den fachlichen Austausch mit Expertinnen und Experten aus Wissenschaft und Praxis pflegen. Die Stiftungskonzepte werden regelmäßig auf Fachtagungen präsentiert und mit Fachleuten anderer Institutionen und Praxisinitiativen diskutiert. Zudem begleitet der Wissenschaftliche Beirat die Stiftung bei Forschungsfragen und bei der fachlichen Fundierung des Stiftungsangebotes. 


\section{Ausblick}

Das Qualitätssystem kann perspektivisch an neue Anforderungen in den Netzwerken oder neue Tätigkeitsbereiche und -profile der Trainerinnen und Trainer angepasst werden, um die Qualität der Fortbildungen für pädagogische Fach- und Lehrkräfte auch bei neuen Schwerpunkten der Bildungsinitiative zu sichern und zu stärken. Neue Anforderungen und Tätigkeitsbereiche für Trainerinnen und Trainer ergeben sich u. a. aus den im Frühjahr 2019 beginnenden Projekten der Stiftung zur Qualitätsentwicklung in Kitas und zu Fortbildungen für den Primarbereich.

Das Projekt PRIMA! wird gefördert vom Bundesministerium für Bildung und Forschung sowie von den beteiligten Bundesländern und zielt auf die Öffnung der Stiftungsangebote für Grundschullehrkräfte im Unterricht. PRIMA! qualifiziert Trainerinnen und Trainer für Grundschulen. Die hierfür erforderlichen Kompetenzen und Fortbildungskonzepte werden auf das erarbeitete Kompetenzmodell für Multiplikatorinnen und Multiplikatoren früher MINT-Bildung aufbauen und bereits entwickelte Weiterbildungsangebote wie Profilfortbildungen sinnvoll nutzen. Dabei wird das Qualitätssystem für Fortbildung angepasst, um die Fortbildungsarbeit mit Grundschullehrkräften angemessen zu berücksichtigen und auch die intensivere Nutzung digitaler Lernangebote zu stärken.

Zur Förderung der pädagogischen Qualität früher MINT-Bildung startet die Stiftung „Haus der kleinen Forscher“ zudem zwei Projekte, die die Qualitätsentwicklung in Kitas aus einer systemischen Perspektive (im Sinne eines Whole Institution Approach, Ferreira, Ryan, \& Tilbury, 2006), auch unter Berücksichtigung der Leitungsebene, in den Blick nehmen. Das erste Projekt Kita-Qualitätsoffensive - Forschen im Alltag (kurz: KiQ-Off) wird vom Bundesministerium für Bildung und Forschung gefördert und im März 2019 starten. Die Qualitätsoffensive hat zum Ziel, einen Beitrag zur Stärkung der pädagogischen Qualität früher MINT-Bildung in Kitas zu leisten und ein wirksames Instrument zur Kompetenz- und Entwicklungsförderung von Kindern zu sein. Es sollen Maßnahmen entwickelt werden, die Kitas bei der Etablierung des forschenden Lernens als pädagogisches Prinzip im Kita-Alltag unterstützen. Das zweite Projekt, eine Initiative zur Kitaentwicklung, das ebenfalls zum März 2019 startet und von der Robert Bosch Stiftung gefördert wird, widmet sich dem Thema Organisationsentwicklung und Qualitätsentwicklung in Kitas. Es zeichnet sich durch eine globale, systemische Herangehensweise an das Thema aus und will übergeordnete Lösungsansätze für das System Kita aus wissenschaftlicher, empirisch-praktischer und bildungspolitischer Sicht erarbeiten. 
Mit diesem verstärkten systemorientierten Bildungsansatz der Stiftung sowie in Bezug auf die Bildungsaktivitäten zur nachhaltigen Entwicklung ist erwartbar, dass insgesamt oder für Teilgruppen von Trainerinnen und Trainern insbesondere der Bereich Organisationswissens für Multiplikatoren in der Initiative an Bedeutung gewinnt. Im Projekt KiQ-Off etwa sollen Prozessbegleitungen (z. B. aus der Gruppe der Trainerinnen und Trainer) qualifiziert werden, um Kitas beim Etablieren des forschenden Lernens in ihrem Alltag professionell zu begleiten und zu unterstützen. Prozessbegleitungen benötigen umfangreiche Beratungskompetenzen und Organisationswissen zum System Kita; diese sollen in einem ersten Schritt auf Grundlage der Zieldimensionen für Multiplikatorinnen und Multiplikatoren genauer beschrieben werden. In einem zweiten Schritt werden entsprechende Qualifizierungsformate für diese Gruppe konzipiert und durchgeführt. Das Projekt KiQ-Off kann bei wichtigen Projektbausteinen wie Beschreibung eines Kompetenzprofils, Auswahl und Qualifizierung von Prozessbegleitungen auf Konzepte, Erfahrungen und geschaffene Strukturen des Qualitätssystems für Fortbildung zurückgreifen.

Diese systemische Herangehensweise und auch die Bildungsaktivitäten zur nachhaltigen Entwicklung werden die Bedeutung des Organisationswissens für Multiplikatorinnen und Multiplikatoren in der Initiative „Haus der kleinen Forscher“ künftig stärken.

Zusammenfassend lässt sich festhalten: Die Zieldimensionen für Multiplikatorinnen und Multiplikatoren in der frühen MINT-Bildung (auch zur Unterstützung einer nachhaltigen Entwicklung) sind Grundlage für ein Qualitätssystem für Fortbildung, das eine bedarfsgerechte Qualifizierung und Unterstützung der Trainerinnen und Trainer und damit auch für die Qualitätssicherung und Qualitätsentwicklung der Fortbildungen für pädagogische Fach- und Lehrkräfte ermöglicht. Dies soll pädagogische Fach- und Lehrkräfte dabei unterstützen, förderliche Lernumgebungen für Kinder zu gestalten, die Freude an Mathematik, Informatik, Naturwissenschaft, Technik und nachhaltiger Entwicklung zu stärken und so zur einer guten Bildung und Entwicklung der Kinder beizutragen. 


\section{Literatur}

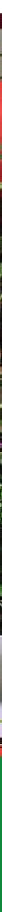




\section{Literatur}

\section{Einleitung -}

Stiftung Haus der kleinen Forscher

Anders, Y. (2013). Stichwort: Auswirkungen frühkindlicher institutioneller Betreuung und Bildung. Zeitschrift für Erziehungswissenschaft, 16(2), S. 237-275.

Anders, Y., \& Ballaschk, I. (2014). Studie zur Untersuchung der Reliabilität und Validität des Zertifizierungsverfahrens der Stiftung „Haus der kleinen Forscher“. In Stiftung Haus der kleinen Forscher (Hrsg.), Wissenschaftliche Untersuchungen zur Arbeit der Stiftung „Haus der kleinen Forscher“" (Band 6, S. 35-116). Schaffhausen: Schubi Lernmedien AG.

Baumert, J., \& Kunter, M. (2006). Stichwort: Professionelle Kompetenz von Lehrkräften. Zeitschrift für Erziehungswissenschaft, 9(4), 469-520.

Beher, K., \& Walter, M. (2012). Qualifikationen und Weiterbildung frühpädagogischer Fachkräfte. Bundesweite Befragung von Einrichtungsleitungen und Fachkräften in Kindertageseinrichtungen: Zehn Fragen-Zehn Antworten. Weiterbildungsinitiative Frühpädagogische Fachkräfte. WiFF Studien, 15.

Blossfeld, H.-P. (2013). Kompetenzentwicklung, Bildungsentscheidungen und Chancenungleichheit in Vorschule und Schule. Neue Ergebnisse aus der Forschung zur Bedeutung von Familien im Bildungssystem. In D. Deißler (Hrsg.), Chancen bilden. Wege zu einer gerechteren Bildung. Ein internationaler Erfahrungsaustausch (S. 37-55). Wiesbaden: Springer.

BMBF (2017). Profis für die Kita. Ergebnisse und Impulse der Forschung zur Aus-, Fort- und Weiterbildung von pädagogischen Fachkräften.

Böhm, J., Jungmann, T., \& Koch, K. (2017). Professionalisierung pädagogischer Fachkräfte. In T. Jungmann \& K. Koch (Hrsg.), Professionalisierung pädagogischer Fachkräfte in Kindertageseinrichtungen. Konzept und Wirksamkeit des KOMPASS-Projektes (S. 9-27). Wiesbaden: Springer.

Buschle, C. (2014). Das professionelle pädagogische Selbstbild von WeiterbildnerInnen frühpädagogischer Fachkräfte (Doctoral dissertation, LMU München).

Fröhlich-Gildhoff, K., Nentwig-Gesemann, I., \& Pietsch, S. (2011). Kompetenzorientierung in der Qualifizierung frühpädagogischer Fachkräfte. Expertise für die Weiterbildungsinitiative.

Fukkink, R. G., \& Lont, A. (2007). Does training matter? A meta-analysis and review of caregiver training studies. Early Childhood Research Quarterly, 22(3), 294-311.

Hippel, A. von (2011). Fortbildung in pädagogischen Berufen - zentrale Themen, Gemeinsamkeiten und Unterschiede der Fortbildung in Elementarbereich, Schule und Weiterbildung. Zeitschrift für Pädagogik, 57, 248-267.

Hippel, A. von, \& Grimm, R. (2010). Qualitätsentwicklungskonzepte in der Weiterbildung Frühpädagogischer Fachkräfte. WiFF Expertise, 3. 
Iller, C. (2015). Kompetenzprofile von Lehrpersonen in der Weiterbildung frühpädagogischer Fachkräfte. In O. Hartungs \& M. Rumpf (Hrsg.), Lehrkompetenzen in der wissenschaftlichen Weiterbildung. Theorie und Empirie Lebenslangen Lernens (S. 89-108). Wiesbaden: Springer.

Jungmann, T., \& Koch, K. (2017). Einleitung. In T. Jungmann \& K. Koch (Hrsg.), Professionalisierung pädagogischer Fachkräfte in Kindertageseinrichtungen. Konzept und Wirksamkeit des KOMPASS-Projektes (S. 1-8). Wiesbaden: Springer.

Kauertz, A., Molitor, H., Saffran, A., Schubert, S., Singer-Brodowski, M., Ulber, D., \& Verch, J. (in Vorbereitung). Zieldimensionen einer Bildung für nachhaltige Entwicklung (BNE) für Kinder, pädagogische Fachkräfte und Leitungskräfte. In Stiftung Haus der kleinen Forscher (Hrsg.), Frühe Bildung für nachhaltige Entwicklung - Ziele und Gelingensbedingungen. Wissenschaftliche Untersuchungen zur Arbeit der Stiftung „Haus der kleinen Forscher“(Band 12). Opladen, Berlin, Toronto: Verlag Barbara Budrich.

Kovacevic, J., \& Nürnberg, C. (2014). Kompetenzorientierung als ein didaktischer Ansatz frühpädagogischer Weiterbildung. Weiterbildungsinitiative Frühpädagogische Fachkräfte. WIFF Studien, 23.

König, A., \& Friederich, T. (2015). Qualität durch Weiterbildung. Konzeptionelle Denkanstöße für die Frühe Bildung. WiFF-Reihe: Perspektive Frühe Bildung.

Lau, M., Fritz, M., \& Hille, K. (2012). Abschlussbericht - Trainerinnen und Trainer im „Haus der kleinen Forscher“. In Stiftung Haus der kleinen Forscher (Hrsg.), Wissenschaftliche Untersuchungen zur Arbeit der Stiftung „Haus der kleinen Forscher“ (Band 3, S. 1382). Köln: Bildungsverlag EINS.

Lencer, S., \& Strauch, A. (2016). Das GRETA-Kompetenzmodell für Lehrende in der Erwachsenen- und Weiterbildung. Abgerufen von www.die-bonn.de/doks/2016-erwachsenenbildung-02.pdf [Stand 22.06.2018].

Müller, M., Faas, S., \& Schmidt-Hertha, B. (2016). Qualitätsmanagement in der frühpädagogischen Weiterbildung. Konzepte, Standards und Kompetenzanerkennung. WiFF Expertise, 45.

Rißmann, M. (2016). Weiterbildung pädagogischer Fachkräfte in Kindertageseinrichtungen. In W. Thole, B. Milbradt, S. Göbel, \& M. Rißmann (Hrsg.), Wissen und Reflexion. Der Alltag in Kindertageseinrichtungen im Blick der Professionellen. (S. 105-130). Wiesbaden: Springer VS.

Starke, S. (2017). Frühpädagogische Professionalisierung - Das Ringen um Anerkennung als professionelles Selbst. In K. Jergus \& C. Thompson (Hrsg.), Autorisierungen des pädagogischen Selbst: Studien zu Adressierungen der Bildungskindheit. (S. 131-173). Wiesbaden: Springer.

Steffensky, M., Anders, Y., Barenthien, J., Hardy, I., Leuchter, M., Oppermann, E., Taskinen, P. \& Ziegler T. (2018). Wirkungen früher naturwissenschaftlicher Bildungsangebote auf die naturwissenschaftlichen Kompetenzen von Fachkräften und Kindern (EASI Science: Early Steps into Science). In Stiftung Haus der kleinen Forscher (Hrsg.), Wirkungen naturwissenschaftlicher Bildungsangebote auf pädagogische Fachkräfte und Kinder. Wissenschaftliche Untersuchungen zur Arbeit der Stiftung „Haus der kleinen Forscher“ (Band 10, S. 50-137). Opladen, Berlin, Toronto: Verlag Barbara Budrich.

Stiftung Haus der kleinen Forscher (Hrsg.) (2013). Wissenschaftliche Untersuchungen zur Arbeit der Stiftung „Haus der kleinen Forscher“ (Band 5). Schaffhausen: Schubi Lernmedien AG. Verfügbar unter: www.haus-der-kleinen-forscher.de. 
Stiftung Haus der kleinen Forscher (2015a). Der pädagogische Ansatz der Stiftung „Haus der kleinen Forscher“. 5. Auflage. Berlin: Stiftung Haus der kleinen Forscher. Verfügbar unter: www.haus-der-kleinen-forscher.de.

Stiftung Haus der kleinen Forscher (Hrsg.) (2015b). Wissenschaftliche Untersuchungen zur Arbeit der Stiftung „Haus der kleinen Forscher“ (Band 7). Schaffhausen: Schubi Lernmedien AG. Verfügbar unter: www.haus-der-kleinen-forscher.de.

Stiftung Haus der kleinen Forscher (2016). DOKU 2016: „Der Forschergeist ist erwacht“. Berlin: Stiftung Haus der kleinen Forscher. Verfügbar unter: www.haus-der-kleinen-forscher.de.

Stiftung Haus der kleinen Forscher (2017a). Monitoring-Bericht 2016/2017 der Stiftung „Haus der kleinen Forscher“. Berlin: Stiftung Haus der kleinen Forscher. Verfügbar unter: www.haus-der-kleinen-forscher.de.

Stiftung Haus der kleinen Forscher (Hrsg.) (2017b). Zertifizierung für Kitas, Horte und Grundschulen. So wird Ihre Einrichtung ein „Haus der kleinen Forscher“. Berlin: Stiftung Haus der kleinen Forscher. Verfügbar unter: www.haus-der-kleinen-forscher.de.

Stiftung Haus der kleinen Forscher (Hrsg.) (2017c). Frühe mathematische Bildung - Ziele und Gelingensbedingungen für den Elementar- und Primarbereich. Wissenschaftiche Untersuchungen zur Arbeit der Stiftung „Haus der kleinen Forscher“(Band 8). Opladen, Berlin, Toronto: Verlag Barbara Budrich. Verfügbar unter: www.haus-der-kleinen-forscher.de.

Stiftung Haus der kleinen Forscher (2018a). Wirkungen naturwissenschaftlicher Bildungsangebote auf pädagogische Fachkräfte und Kinder. Wissenschaftliche Untersuchungen zur Arbeit der Stiftung „Haus der kleinen Forscher“(Band 10). Opladen, Berlin, Toronto: Verlag Barbara Budrich. Verfügbar unter: www.haus-der-kleinen-forscher.de.

Stiftung Haus der kleinen Forscher (Hrsg.) (2018b). Frühe informatische Bildung - Ziele und Gelingensbedingungen für den Elementar- und Primarbereich. Wissenschaftliche Untersuchungen zur Arbeit der Stiftung „Haus der kleinen Forscher“(Band 9). Opladen, Berlin, Toronto: Verlag Barbara Budrich. Verfügbar unter: www.haus-der-kleinen-forscher.de.

Stiftung Haus der kleinen Forscher (2019). Der pädagogische Ansatz der Stiftung „Haus der kleinen Forscher“. 6. Auflage. Berlin: Stiftung Haus der kleinen Forscher. Verfügbar unter: www.haus-der-kleinen-forscher.de.

Stiftung Haus der kleinen Forscher (in Vorbereitung). Frühe Bildung für nachhaltige Entwicklung - Ziele und Gelingensbedingungen. Wissenschaftliche Untersuchungen zur Arbeit der Stiftung „Haus der kleinen Forscher“ (Band 12). Opladen, Berlin, Toronto: Verlag Barbara Budrich. Verfügbar unter: www.haus-der-kleinen-forscher.de.

Tietze, W., Roßbach, H.-G., \& Grenner, K. (2005). Kinder von 4 bis 8 Jahren. Zur Qualität der Erziehung und Bildung im Kindergarten, Grundschule und Familie. Weinheim: Beltz.

Tippelt, R., Hippel, A. von, \& Fuchs, S. (2009). Fortbildung der Weiterbildner/innen als Grundlage für Qualität. In A. von Hippel \& R. Tippelt (Hrsg.), Fortbildung der WeiterbildnerInnen - eine Analyse der Interessen und Bedarfe aus verschiedenen Perspektiven (S. 11-17). Weinheim: Beltz.

Wahl, D. (2013). Lernumgebungen erfolgreich gestalten: Vom trägen Wissen zum kompetenten Handeln. Bad Heilbrunn: Julius Klinkhardt.

Wißhak, S. (2017). Qualifizierung und professionelles Wissen von Lehrpersonen in der berufsbezogenen Weiterbildung (Dissertation, Universität Konstanz). 


\section{Zieldimensionen für Multiplikatorinnen und Multiplikatoren früher MINT-Bildung -}

Olaf Köller, Johannes Magenheim, Uwe Pfenning, Jörg Ramseger, Mirjam

Steffensky, Christian Wiesmüller, Esther Winther, Bernd Wollring

Ambos, I., Egetenmeyer, R., \& Scheller, F. (2008). wbmonitor 2008: Personalentwicklung und wirtschaftliches Klima bei Weiterbildungsanbietern. Bonn: Bundesinstitut für Berufsbildung und Deutsches Institut für Erwachsenenbildung.

Anders, Y. (2013). Stichwort: Auswirkungen frühkindlicher institutioneller Betreuung und Bildung. Zeitschrift für Erziehungswissenschaft, 16(2), 237-275.

Anders, Y., Hardy, I., Pauen, S., \& Steffensky, M. (2013). Zieldimensionen naturwissenschaftlicher Bildung im Kita-Alter und ihre Messung. In Stiftung Haus der kleinen Forscher (Hrsg.), Wissenschaftliche Untersuchungen zur Arbeit der Stiftung „Haus der kleinen Forscher" (Band 5, S. 19-82). Schaffhausen: Schubi Lernmedien.

Anders, Y., Hardy, I., Sodian, B., \& Steffensky, M. (2013). Zieldimensionen naturwissenschaftlicher Bildung im Grundschulalter und ihre Messung. In Stiftung Haus der kleinen Forscher (Hrsg.), Wissenschaftliche Untersuchungen zur Arbeit der Stiftung „Haus der kleinen Forscher" (Band 5, S. 83-164). Schaffhausen: Schubi Lernmedien.

Arnold, R., \& Siebert, H. (1995). Konstruktivistische Erwachsenenbildung. Von der Deutung zur Konstruktion von Wirklichkeit. Baltmannsweiler: Schneider.

Barzel, B., \& Selter, C. (2015). Die DZLM-Gestaltungsprinzipien für Fortbildungen. Journal für Mathematik-Didaktik, 36 (2), 259-284.

Bastian, H. (1997). Kursleiterprofile und Angebotsqualität. Bad Heilbrunn: Klinkhardt.

Baumert, J., \& Kunter, M. (2006). Stichwort: Professionelle Kompetenz von Lehrkräften. Zeitschrift für Erziehungswissenschaft, 9(4), 469-520.

Baumert, J., \& Kunter, M. (2011). Das Kompetenzmodell von COACTIV. In M. Kunter, J. Baumert, W. Blum, U. Klusmann, S. Krauss, \& M. Neubrand (Hrsg.), Professionelle Kompetenz von Lehrkräften - Ergebnisse des Forschungsprogramms COACTIV (S. 29-53). Münster: Waxmann.

Benz, C., Grüßing, M., Lorenz, J. H., Selter, C., \& Wollring, B. (2017). Zieldimensionen mathematischer Bildung im Elementar- und Primarbereich. In Stiftung Haus der kleinen Forscher (Hrsg.), Frühe mathematische Bildung - Ziele und Gelingensbedingungen für den Elementar- und Primarbereich. Wissenschaftliche Untersuchungen zur Arbeit der Stiftung „Haus der kleinen Forscher“ (Band 8, S. 32-177). Opladen, Berlin, Toronto: Verlag Barbara Budrich.

Bergner, N., Köster, H., Magenheim, J., Müller, K., Romeike, R., Schröder, U., \& Schulte, C. (2018). Zieldimensionen informatischer Bildung im Elementar- und Primarbereich. In Stiftung Haus der kleinen Forscher (Hrsg.), Frühe informatische Bildung - Ziele und Gelingensbedingungen für den Elementar- und Primarbereich. Wissenschaftliche Untersuchungen zur Arbeit der Stiftung „Haus der kleinen Forscher“ (Band 9, S. 38-267). Opladen, Berlin, Toronto: Verlag Barbara Budrich.

Bilger (2013). Weiterbildungsverhalten in Deutschland: AES 2012 Trendbericht. Bonn: Bundesministerium für Bildung und Forschung. 
Blömeke, S., Jenßen, L., Dunekacke, S., Suhl, U., Grassmann, M., \& Wedekind, H. (2015). Leistungstests zur Messung der professionellen Kompetenz frühpädagogischer Fachkräfte. Zeitschrift für pädagogische Psychologie, 29, 177-191.

Blömeke, S., Kaiser, G., \& Lehmann, R. (Hrsg.) (2010). TEDS-M 2008 - Professionelle Kompetenz und Lerngelegenheiten angehender Primarstufenlehrkräfte im internationalen Vergleich. Münster: Waxmann.

Brandt, P. (2011). Stichwort: Stiefkind Fachdidaktik. DIE - Zeitschrift für Erwachsenenbildung, 18, 20-21.

Bromme, R. (1992). Der Lehrer als Experte. Bern: Huber.

Burger, K. (2010). How does early childhood care and education affect cognitive development? An international review of the effects of early interventions for children from different social backgrounds. Early childhood research quarterly, 25(2), 140-165.

Egetenmeyer, R. \& Strauch, A. (2009). Adult educators in Germany: Challenges towards professionalisation in Europe. Journal of Educational Sciences/Revista de Stiintele Educatiei, 11(1), 87-94.

Gruber, H. (2004). Kompetenzen von Lehrerinnen und Lehrern - Ein Blick aus der Expertiseforschung (Forschungsbericht Nr. 13). Regensburg: Universität Regensburg.

Hartz, S. (2011). Qualität. In K. P. Horn, H. Kemnitz, W. Marotzki, \& U. Sandfuchs (Hrsg.), Lexikon der Erziehungswissenschaft (S. 51-52). Bad Heilbrunn: Klinkhardt.

Heid, H. (2000). Über die Qualität der Argumente, mit denen das Erfordernis lebenslangen Lernens begründet wird. In F. Achtenhagen, \& W. Lempert, (Hrsg.), Lebenslanges Lernen im Beruf - seine Grundlegung im Kindes- und Jugendalter. Band 5: Erziehungstheorie und Bildungsforschung (S. 22-29). Opladen : Leske + Budrich.

Hof, C. (2001). Konzepte des Wissens. Eine empirische Studie zu den wissenstheoretischen Grundlagen des Unterrichtens. Bielefeld: Bertelsmann.

Holzkamp, K. (1993). Lernen: Subjektwissenschaftliche Grundlegung. Stuttgart: Campus-Verlag.

Kade, S. (1990). Handlungshermeneutik: Qualifizierung durch Fallarbeit. Bad Heilbrunn/ Obb: Julius Klinkhardt.

Kade, S. (1990). Handlungshermeneutik: Qualifizierung durch Fallarbeit. Julius Klinkhardt.

KMK (2004). Standards für die Lehrerbildung: Bildungswissenschaften. Beschluss der Kultusministerkonferenz vom 16.12.2004. Berlin: Sekretariat der Ständigen Konferenz der Kultusminister der Länder in der Bundesrepublik Deutschland.

KMK, \& JMK (2004). Gemeinsamer Rahmen der Länder für die frühe Bildung in Kindertageseinrichtungen. Bonn: Beta Verlag \& Marketinggesellschaft mbH.

Kosack, W., Jeretin-Kopf, M., \& Wiesmüller, C. (2015). Zieldimensionen technischer Bildung im Elementar- und Primarbereich. In Stiftung Haus der kleinen Forscher (Hrsg.), Wissenschaftliche Untersuchungen zur Arbeit der Stiftung „Haus der kleinen Forscher“ (Band 7, S. 30-157). Schaffhausen: Schubi Lernmedien AG.

Kosubek, T., Schwickerath, A., Kollmannsberger, M., \& Hippel, A. von (2009). Fortbildungsmotivation und Barrieren. In A. von Hippel, \& R. Tippelt (Hrsg.), Fortbildung der Weiterbildner/innen - eine Analyse der Interessen und Bedarfe aus verschiedenen Perspektiven (S. 114-142). Weinheim: Beltz.

Kraft, S., \& Seitter, W. (2008). Konstruktive Annäherungen an EQF und DQR. Das Modell „Grund- und Fortbildungszertifikat für Lehrende in der Weiterbildung“. DIE - Zeitschrift für Erwachsenenbildung, 15(4), 39-42. 
Kunter, M., Kleickmann, T., Klusmann, U., \& Richter, D. (2011). Die Entwicklung professioneller Kompetenz von Lehrkräften. Professionelle Kompetenz von Lehrkräften. Ergebnisse des Forschungsprogramms COACTIV, 1, 55-68.

Kunter, M., Klusmann, U., Baumert, J., Richter, D., Voss, T., \& Hachfeld, A. (2013). Professional competence of teachers: Effects on instructional quality and student development. Journal of Educational Psychology, 105(3), 805-820.

Lencer, S., \& Strauch, A. (2016). Das GRETA-Kompetenzmodell für Lehrende in der Erwachsenen- und Weiterbildung. Abgerufen von www.die-bonn.de/doks/2016-erwachsenenbildung-02.pdf [Stand 22.06.2018].

Leser, I., Mey, G., Mruck, K., Ramseger, J., \& Vock, R. (2011). prima(r)forscher. Naturwissenschaftliches Lernen im Grundschulnetzwerk. Abschlussbericht der wissenschaftlichen Begleitung. Bonn, Berlin: Deutsche Telekom Stiftung und Deutsche Kinder- und Jugendstiftung.

Lipowsky, F., \& Rzejak, D. (2012). Lehrerinnen und Lehrer als Lerner - Wann gelingt der Rollentausch? Merkmale und Wirkungen wirksamer Lehrerfortbildungen. Schulpädagogik heute, 3 (5), 1-17.

Lipowsky, F., \& Rzejak, D. (2015a). Lehrerfortbildungen lernwirksam gestalten - Ein Überblick über den Forschungsstand. ZfL Magazin der Universität Münster, 1(1), 5-10.

Lipowsky, F., \& Rzejak, D. (2015b). Was wir über gelingende Lehrerfortbildungen wissen. Journal für LehrerInnenbildung, 15(4), 26-32.

Martin, A., \& Langemeyer, I. (2014). Demographie, sozioökonomischer Status und Stand der Professionalisierung - das Personal der Weiterbildung im Vergleich. In Deutsches Institut für Erwachsenenbildung (Hrsg.), Trends der Weiterbildung: DIE-Trendanalyse 2014 (S. 43-88). Bielefeld: Bertelsmann.

NICHD Early Child Care Research Network. (2002). Child-care structure....; process $\cdots \cdot ;$ outcome: Direct and indirect effects of child-care quality on young children's development. Psychological science, 13(3), 199-206.

Nittel, D. (2000). Von der Mission zur Profession? Stand und Perspektiven der Verberuflichung in der Erwachsenenbildung. Bielefeld: Bertelsmann.

Nittel, D. (2006). Das „Haus des lebenslangen Lernens in Dreieich“ - Eine innovative Organisation des Bildungswesens. Hessische Blätter für Volksbildung, 3, 247-259.

Richter, D., Kuhl, P., Haag, N., \& Pant, H. A. (2012). Aspekte der Aus-und Fortbildung von Mathematik- und Naturwissenschaftslehrkräften im Ländervergleich. IQB-Ländervergleich, 367-390.

Ropohl, G. (1979). Eine Systemtheorie der Technik. München und Wien.

Rosenbladt, B. von, \& Thebis, F. (Hrsg.) (2004). Berufliche und soziale Lage von Lehrenden in der Weiterbildung. Berlin: Bundesministerium für Bildung und Forschung.

Roßbach, H. G. (2005). Effekte qualitativ guter Betreuung, Bildung und Erziehung im frühen Kindesalter auf Kinder und ihre Familien. In Sachverständigenkommission Zwölfter Kinder- und Jugendbericht (Hrsg.), Bildung, Betreuung und Erziehung von Kindern unter sechs Jahren. Materialien zum 12. Kinder- und Jugendbericht (S. 55-174). München: Deutsches Jugendinstitut.

Rotthaus, W. (2001): Wozu erziehen? Entwurf einer systemischen Erziehung. Heidelberg: Carl-Auer-Systeme Verlag. 
Schöb, S., Sahlender, M., Brandt, P., Fischer, M., \& Wintermann, O. (2015). Information und Vernetzung - Bedarfe und Erwartungen von Lehrkräften an online-gestützte Fortbildungsangebote. Bonn: Deutsches Institut für Erwachsenenbildung.

Schrader, J., \& Goeze, A. (2016). Professionelle Kompetenzen von Lehrkräften in der Erwachsenen- und Weiterbildung - Ein Rahmenmodell für Forschung, Rekrutierung und Fortbildung. Manuskript in Vorbereitung.

Spelke, E. (1994). Initial knowledge: Six suggestions. Cognition, 50(1-3), 431-445.

Sylva, Sammons, Chan, Melhuish, Siraj-Blatchford, \& Taggart (2013). Sylva, K., Sammons, P., Chan, L. L., Melhuish, E., Siraj-Blatchford, I., \& Taggart, B. (2013). The effects of early experiences at home and pre-school on gains in English and mathematics in primary school: a multilevel study in England. Zeitschrift für Erziehungswissenschaft, 16(2), 277-301.

Tietgens, H. (1988). Professionalität für die Erwachsenenbildung. In W. Gieseke (Hrsg.), Professionalität und Professionalisierung (S. 28-75). Bad Heilbrunn/Obb.: Julius Klinkhardt.

Tippelt, R., \& Hippel, A. von (2007). Kompetenzförderung von ErwachsenenbildnerInnen im Bereich Teilnehmer-, Adressaten- und Milieuorientierung als Beitrag zur Professionalität in der Erwachsenenbildung. In U. Heuer, \& R. Siebers (Hrsg.), Weiterbildung am Beginn des 21. Jahrhunderts. Festschrift für Wiltrud Gieseke (S. 118-129). Münster: Waxmann.

Weinert, S., Doil, H., \& Frevert, S. (2008). Bildungsforschung. Kindliche Kompetenzen im Elementarbereich: Förderbarkeit, Bedeutung und Messung. Berlin: Bundesministerium für Bildung und Forschung.

Wiesner, G. (2009). Kompetenzpass für Weiterbildner/innen. Erwachsenenpädagogische Kompetenzen bilanzieren. Dresden: Technische Universität Dresden.

Wigger, L. (2004). Didaktik. In D. Benner, \& J. Oelkers (Hrsg.), Historisches Wörterbuch der Pädagogik (S. 244-278). Weinheim und Basel: Beltz.

WSF (2005). Erhebung zur beruflichen und sozialen Lage von Lehrenden in Weiterbildungseinrichtungen: Schlussbericht. Kerpen: Bundesministerium für Bildung und Forschung.

\section{Zieldimensionen für Multiplikatorinnen und Multiplikatoren früher MINT-Bildung aus Sicht einer Bildung für nachhaltige Entwicklung -}

Heike Molitor

Davis, J. M. (2012, June). ESD Starts Where STEM Stops: Integrating the Social Sciences into STEM. Paper presented at the 2nd International STEM in Education Conference, Athens, Greece. Abgerufen von http://citeseerx.ist.psu.edu/viewdoc/download;jsessionid=DD673589960A60D3285CCCDE1D1FF8FD?doi=10.1.1.663.7578\&rep=rep1 \&type $=$ pdf [Stand: 20.08.2018].

Deutsche UNESCO-Kommission (2014). UNESCO Roadmap zur Umsetzung des Weltaktionsprogramms „Bildung für nachhaltige Entwicklung“. Bonn: Deutsche UNESCO-Kommission. 
Ferreira, J., Ryan, L., \& Tilbury, D. (2006). Whole-School Approaches to Sustainability: A review of models for professional development in pre-service teacher education. Canberra: Australian Government Department of the Environment and Heritage and the Australian Research Institute in Education for Sustainability (ARIES).

Gesellschaft für Didaktik des Sachunterrichts (Hrsg.) (2002). Perspektivrahmen Sachunterricht. Bad Heilbrunn: Klinkhardt.

Gesellschaft für Didaktik des Sachunterrichts (Hrsg.) (2013). Perspektivrahmen Sachunterricht. Bad Heilbrunn: Klinkhardt.

Kauertz, A., Molitor, H., Saffran, A., Schubert, S., Singer-Brodowski, M., Ulber, D., \& Verch, J. (in Vorbereitung). Zieldimensionen einer Bildung für nachhaltige Entwicklung (BNE) für Kinder, pädagogische Fachkräfte und Leitungskräfte. In Stiftung Haus der kleinen Forscher (Hrsg.), Frühe Bildung für nachhaltige Entwicklung - Ziele und Gelingensbedingungen. Wissenschaftliche Untersuchungen zur Arbeit der Stiftung „Haus der kleinen Forscher"(Band 12). Opladen, Berlin, Toronto: Verlag Barbara Budrich.

Molitor, H., \& Ibisch, P. L. (2018). Nachhaltigkeit als Reaktion: Was bisher geschah. In P. L. Ibisch, H. Molitor, A. Conrad, H. Walk, V. Mihotovic, \& J. Geyer (Hrsg.), Der Mensch im globalen Ökosystem. Eine Einführung in die nachhaltige Entwicklung (S. 35-58). München: oekom.

Rathgeber, M. (2012): Energie - ein Thema für den Kindergarten? In S. Schubert, Y. Salewski, E. Späth, \& A. Steinberg (Hrsg.), Nachhaltigkeit entdecken, verstehen, gestalten. Kindergärten als Bildungsorte nachhaltiger Entwicklung (S. 64-69). Weimar, Berlin: das netz.

Schubert, S., Salewski, Y., Späth, E., \& Steinberg, A. (2013). Was macht die Kuh im Kühlschrank? Bildung für nachhaltige Entwicklung im Zuhause und in der Kita. Weimar, Berlin: das netz.

\section{Fazit und Ausblick -}

\section{Stiftung Haus der kleinen Forscher}

Buhl, M., Buschle, C., Freytag, T., Hippel, A. von, \& Iller, C. (2014). Kompetenzorientierung in der Weiterbildung: gemeinsame Aufgabe in verteilten Zuständigkeiten. In DJI (Hrsg.), Kompetenzorientierte Gestaltung von Weiterbildungen. Grundlagen für die Frühpädagogik (S. 54-69). WiFF Wegweiser, Band 7. München: DJI.

Buschle, C. (2014). Das professionelle pädagogische Selbstbild von WeiterbildnerInnen frühpädagogischer Fachkräfte (Doctoral dissertation, LMU München).

Ferreira, J., Ryan, L., \& Tilbury, D. (2006). Whole-School Approaches to Sustainability: A review of models for professional development in pre-service teacher education. Canberra: Australian Government Department of the Environment and Heritage and the Australian Research Institute in Education for Sustainability (ARIES).

Fröhlich-Gildhoff, K., Nentwig-Gesemann, I., \& Pietsch, S. (2011). Kompetenzorientierung in der Qualifizierung frühpädagogischer Fachkräfte. Expertise für die Weiterbildungsinitiative.

Gesellschaft für Didaktik des Sachunterrichts (Hrsg.) (2002). Perspektivrahmen Sachunterricht. Bad Heilbrunn: Klinkhardt. 
Gesellschaft für Didaktik des Sachunterrichts (Hrsg.) (2013). Perspektivrahmen Sachunterricht. Bad Heilbrunn: Klinkhardt.

Iller, C. (2015). Kompetenzprofile von Lehrpersonen in der Weiterbildung frühpädagogischer Fachkräfte. In O. Hartungs \& M. Rumpf (Hrsg.), Lehrkompetenzen in der wissenschaftlichen Weiterbildung. Theorie und Empirie Lebenslangen Lernens (S. 89-108). Wiesbaden: Springer.

Krüger, T, \& Laubach, B. (2018). Nutzung der Online-Lernangebote der Stiftung „Haus der kleinen Forscher". Bonn: Umfragezentrum Bonn.

Marquardt-Mau, B. (2011). Der Forschungskreislauf: Was bedeutet forschen im Sachunterricht? In Deutsche Telekom Stiftung und Deutsche Kinder- und Jugendstiftung (Hrsg.), Wie gute naturwissenschaftliche Bildung an Grundschulen gelingt. Ergebnisse und Erfahrungen aus Primarforscher. Berlin: DKJS.

Müller, M., Faas, S., \& Schmidt-Hertha, B. (2016). Qualitätsmanagement in der frühpädagogischen Weiterbildung. Konzepte, Standards und Kompetenzanerkennung. WiFF Expertise, 45.

Schwarzer, R., \& Jerusalem, M. (Hrsg.) (1999). Skalen zur Erfassung von Lehrer- und Schülermerkmalen. Dokumentation der psychometrischen Verfahren im Rahmen der wissenschaftlichen Begleitung des Modellversuchs Selbstwirksame Schulen. Berlin: Freie Universität Berlin.

Starke, S. (2017). Frühpädagogische Professionalisierung - Das Ringen um Anerkennung als professionelles Selbst. In K. Jergus \& C. Thompson (Hrsg.), Autorisierungen des pädagogischen Selbst: Studien zu Adressierungen der Bildungskindheit (S. 131-173). Wiesbaden: Springer.

Steffensky, M., Anders, Y., Barenthien, J., Hardy, I., Leuchter, M., Oppermann, E., Taskinen, P., \& Ziegler T. (2018). Wirkungen früher naturwissenschaftlicher Bildungsangebote auf die naturwissenschaftlichen Kompetenzen von Fachkräften und Kindern (EASI Science: Early Steps into Science). In Stiftung Haus der kleinen Forscher (Hrsg.), Wirkungen naturwissenschaftlicher Bildungsangebote auf pädagogische Fachkräfte und Kinder. Wissenschaftliche Untersuchungen zur Arbeit der Stiftung „Haus der kleinen Forscher“ (Band 10, S. 50-137). Opladen, Berlin, Toronto: Verlag Barbara Budrich.

Stiftung Haus der kleinen Forscher (Hrsg.) (2013). Wissenschaftliche Untersuchungen zur Arbeit der Stiftung „Haus der kleinen Forscher“ (Band 5). Schaffhausen: Schubi Lernmedien AG. Verfügbar unter: www.haus-der-kleinen-forscher.de.

Stiftung Haus der kleinen Forscher (2015a). Der pädagogische Ansatz der Stiftung „Haus der kleinen Forscher“. 5. Auflage. Berlin: Stiftung Haus der kleinen Forscher. Verfügbar unter: www.haus-der-kleinen-forscher.de.

Stiftung Haus der kleinen Forscher (Hrsg.) (2015b). Wissenschaftliche Untersuchungen zur Arbeit der Stiftung „Haus der kleinen Forscher“ (Band 7). Schaffhausen: Schubi Lernmedien AG. Verfügbar unter: www.haus-der-kleinen-forscher.de.

Stiftung Haus der kleinen Forscher (Hrsg.) (2017c). Frühe mathematische Bildung - Ziele und Gelingensbedingungen für den Elementar- und Primarbereich. Wissenschaftliche Untersuchungen zur Arbeit der Stiftung „Haus der kleinen Forscher“ (Band 8). Opladen, Berlin, Toronto: Verlag Barbara Budrich. Verfügbar unter: www.haus-der-kleinen-forscher.de. Verfügbar unter: www.haus-der-kleinen-forscher.de. 
Stiftung Haus der kleinen Forscher (Hrsg.) (2018b). Frühe informatische Bildung - Ziele und Gelingensbedingungen für den Elementar- und Primarbereich. Wissenschaftliche Untersuchungen zur Arbeit der Stiftung „Haus der kleinen Forscher“(Band 9). Opladen, Berlin, Toronto: Verlag Barbara Budrich. Verfügbar unter: www.haus-der-kleinen-forscher.de.

Stiftung Haus der kleinen Forscher (2019). Der pädagogische Ansatz der Stiftung „Haus der kleinen Forscher“. 6. Auflage. Berlin: Stiftung Haus der kleinen Forscher. Verfügbar unter: www.haus-der-kleinen-forscher.de.

Stiftung Haus der kleinen Forscher (in Vorbereitung). Frühe Bildung für nachhaltige Entwicklung - Ziele und Gelingensbedingungen. Wissenschaftliche Untersuchungen zur Arbeit der Stiftung „Haus der kleinen Forscher“ (Band 12). Opladen, Berlin, Toronto: Verlag Barbara Budrich. Verfügbar unter: www.haus-der-kleinen-forscher.de.

Wedel, A., \& Fritz, M. (2018). Qualitative Studie „Kleine Forscher“-Kompass. Akzeptanz und subjektiver Nutzen des Online-Tools im Haus der kleinen Forscher (Forschungsbericht). Berlin und Dresden: Technische Universität Berlin, IRIS e. V.

Wißhak, S. (2017). Qualifizierung und professionelles Wissen von Lehrpersonen in der berufsbezogenen Weiterbildung (Dissertation, Universität Konstanz). 



\section{Anhang}

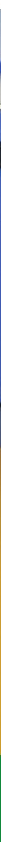




\section{Anhang: Zieldimensionen für Multiplikatorinnen und Multiplikatoren früher MINT-Bildung}

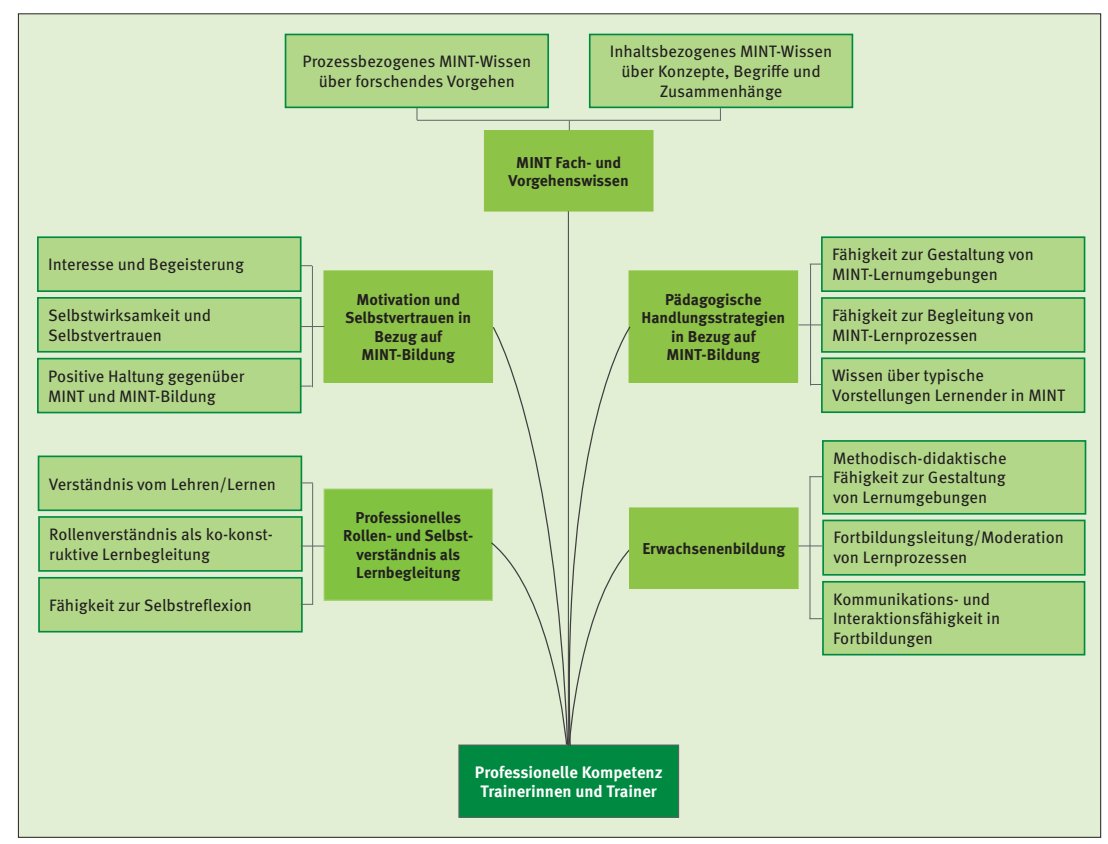

Quelle: Stiftung Haus der kleinen Forscher (Hrsg.); nach Köller et al. 2019, In: Zieldimensionen für Multiplikatorinnen und Multiplikatoren früher MINT-Bildung. Wissenschaftliche Untersuchungen zur Arbeit der Stiftung „Haus der kleinen Forscher“ (Band 11) 


\section{Bildquellenverzeichnis}

Seite 44, 48, 124, 136: (C) Thomas Ernst/Stiftung „Haus der kleinen Forscher“ Seite 12: () Bettina Volke/Stiftung „Haus der kleinen Forscher“

Seite 22: ( B Birte Filmer/Stiftung „Haus der kleinen Forscher“

Seite 75, 98: @ Frank Bentert/Stiftung „Haus der kleinen Forscher“

Seite 86: () Stiftung „Haus der kleinen Forscher“ 


\section{Über die Stiftung „Haus der kleinen Forscher“}

Die gemeinnützige Stiftung „Haus der kleinen Forscher“ engagiert sich für gute frühe Bildung in den Bereichen Mathematik, Informatik, Naturwissenschaften und Technik (MINT) - mit dem Ziel, Mädchen und Jungen stark für die Zukunft zu machen und zu nachhaltigem Handeln zu befähigen. Gemeinsam mit ihren Netzwerkpartnern vor Ort bietet die Stiftung bundesweit ein Bildungsprogramm an, das pädagogische Fach- und Lehrkräfte dabei unterstützt, Kinder im Kita- und Grundschulalter qualifiziert beim Entdecken, Forschen und Lernen zu begleiten. Das „Haus der kleinen Forscher“ verbessert Bildungschancen, fördert Interesse am MINT-Bereich und professionalisiert dafür pädagogisches Personal. Partner der Stiftung sind die Helmholtz-Gemeinschaft, die Siemens Stiftung, die Dietmar Hopp Stiftung und die Deutsche Telekom Stiftung. Gefördert wird sie vom Bundesministerium für Bildung und Forschung.

\section{Vision und Mission der Stiftung „Haus der kleinen Forscher"6 \\ Vision der Stiftung „Haus der kleinen Forscher“: \\ Fragen - Forschen - Zukunft gestalten}

Alle Kinder in Deutschland erleben Bildungsorte, in denen sie ihren eigenen Fragen nachgehen und forschend die Welt entdecken können. Solche „Häuser der kleinen Forscher“ machen Mädchen und Jungen stark für die Zukunft. Sie befähigen Kinder, selbstbestimmt zu denken und verantwortungsvoll zu handeln.

Technologisierung und Digitalisierung sowie Folgen des Klimawandels und der sozialen Ungleichheit beeinflussen zunehmend unseren Alltag. Wir tragen dazu bei, dass sich Menschen in unserer schnell verändernden Welt orientieren können und offen für Neues bleiben.

Die alltägliche Auseinandersetzung mit Natur und Technik fördert Neugier, Lern- und Denkfreude der Mädchen und Jungen. Wir sehen frühe Bildung als Schlüssel, um den Herausforderungen einer komplexen Welt erfolgreich begegnen zu können. 


\section{Mission der Stiftung „Haus der kleinen Forscher“:}

Die Stiftung „Haus der kleinen Forscher“ ”...

befördert eine fragend-forschende Haltung bei Kindern,

- gibt Mädchen und Jungen schon in jungen Jahren die Chance, eigene Talente und Potenziale in den Bereichen Naturwissenschaften, Technik, Mathematik und Informatik zu entdecken

und legt den Grundstein für einen reflektierten Umgang mit technologischen und gesellschaftlichen Veränderungen im Sinne einer nachhaltigen Entwicklung.

Gemeinsam mit ihren Bezugspersonen erleben die Kinder Spaß und Freude am Entdecken und Verstehen dieser Welt. Kinder gestalten Bildungsprozesse aktiv mit und erleben sich dadurch als kompetent und selbstwirksam in ihrem Alltag. Beim forschenden Lernen können Kinder Problemlösekompetenzen entwickeln, eigene Antworten finden und Selbstvertrauen spüren („Ich kann!“) - Erfahrungen und Fähigkeiten, die weit über die Kindheit hinaus für die Persönlichkeitsentwicklung und die spätere Berufsbiografie von Bedeutung sind.

In einem praxisnahen und qualitativ hochwertigen Professionalisierungsansatz unterstützt die Stiftung pädagogische Fach- und Lehrkräfte dabei, Kinder im Alter bis 10 Jahren beim Entdecken, Forschen und Lernen zu begleiten. Über vielfältige Fortbildungsangebote erleben Fach- und Lehrkräfte die Faszination eigenen Forschens für sich selbst. Sie erweitern ihre Kenntnisse und pädagogischen Kompetenzen und setzen sie in ihrer alltäglichen Arbeit mit Kindern um.

Die Initiative unterstützt Bildungseinrichtungen darin, sich als „Ort des forschenden Lernens“ nachhaltig weiterzuentwickeln und in diesem Sinn als „Haus der kleinen Forscher“ förderliche Lernumgebungen für Kinder zu schaffen. 


\section{Bisher erschienen in der Wissenschaftlichen Schriftenreihe der Stiftung „Haus der kleinen Forscher“}

Band 1 (2011)

Dagmar Berwanger, Petra Evanschitzky, Elke Heller, Christa Preissing, Ursula Rabe-Kleberg, Franziska Schulze, Anna Spindler

Der erste Band der Schriftenreihe „Wissenschaftliche Untersuchungen zur Arbeit der Stiftung ,Haus der kleinen Forscher““ stellt vier wissenschaftliche Expertisen aus den Jahren 2009 und 2010 vor, die von renommierten Wissenschaftlerinnen und Wissenschaftlern aus dem Bereich der frühkindlichen Pädagogik verfasst wurden. Die vorliegenden wissenschaftlichen Beiträge reflektieren den pädagogischen Ansatz und das Multiplikatorenmodell der Stiftung vor dem Hintergrund der eigenen Zielvorstellungen, analysieren die Umsetzungspraxis in den Netzwerken und Kitas und zeigen weitere Entwicklungsmöglichkeiten auf.

\section{Band 2 (2011)}

\section{Franziska Kramer, Ursula Rabe-Kleberg}

Die Studie von Ursula Rabe-Kleberg und Franziska Kramer bildet eine logische Konsequenz und Ergänzung zur ersten Untersuchung der Autorinnen „Erzieherinnen und ihre Haltung zu Naturwissenschaft und Technik für Jungen und Mädchen“, die in Band 1 dieser Schriftenreihe veröffentlicht wurde (siehe oben). Die Autorinnen untersuchen die Gestaltung der Lernprozesse durch Erzieherinnen im Detail. Mit hoher Präzision und Sensibilität im Umgang mit den Möglichkeiten qualitativer Sozialforschung gelingt es den Autorinnen dabei, ko-konstruktive Augenblicke des gemeinsamen Lernens in Kitas einzufangen und intensiv im Hinblick auf wichtige Einflussvariablen zu reflektieren.
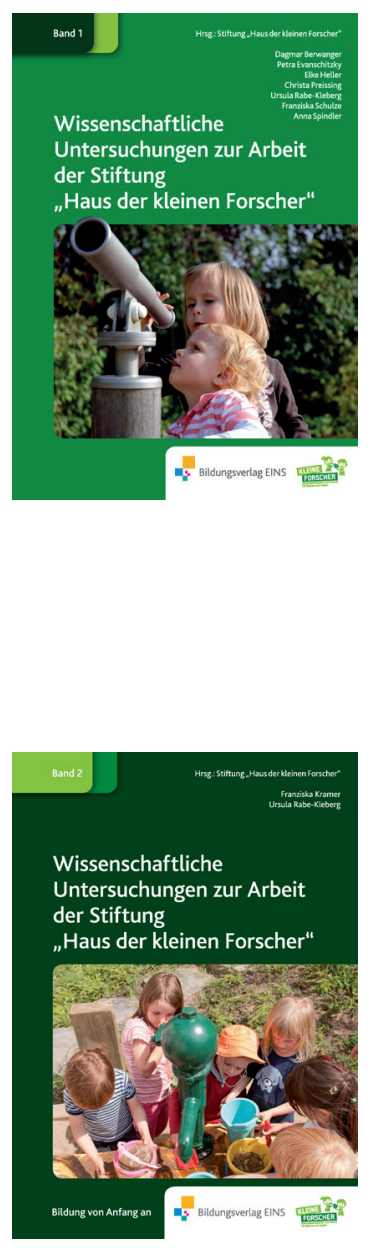


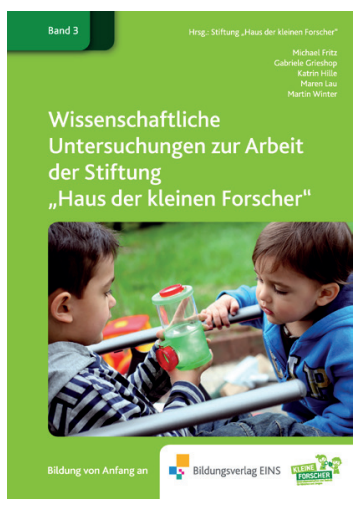

Band 3 (2012)

Michael Fritz, Gabriele Grieshop, Katrin Hille, Maren Lau, Martin Winter

Im dritten Band werden zwei Studien vorgestellt, die sich mit der Rolle der Bedeutung der Trainerinnen und Trainer in der Initiative „Haus der kleinen Forscher“ aus jeweils unterschiedlichen Perspektiven beschäftigen: In der Studie von Maren Lau, Michael Fritz und Katrin Hille (ZNL) stehen das Rollen- und Selbstverständnis der Trainerinnen und Trainer sowie ihr subjektives Kompetenzempfinden im Mittelpunkt. In der Untersuchung von Gabriele Grieshop und Martin Winter (Institut für Didaktik der Mathematik und des Sachunterrichts (IFD), Universität Vechta) wird - im Rahmen einer vorwiegend formativen Implementierungsevaluation am Beispiel Mathematik - die Beteiligung der Trainerinnen und Trainer an der Konzept- und Materialentwicklung von Angeboten der Stiftung „Haus der kleinen Forscher" betrachtet.

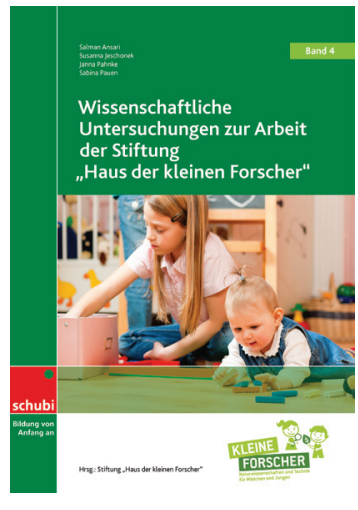

Band 4 (2012)

Salman Ansari, Susanna Jeschonek, Janna Pahnke, Sabina Pauen

Band 4 enthält vier Expertisen, die basierend auf aktuellen entwicklungspsychologischen Erkenntnissen Empfehlungen für die Entwicklung weiterer naturwissenschaftlicher, technischer und mathematischer Themenschwerpunkte der Stiftung „Haus der kleinen Forscher“ aussprechen, auf mögliche Stolpersteine hinweisen und Vorschläge für die Praxis aufzeigen. Die Expertise von Janna Pahnke und Sabina Pauen gibt einen Überblick über die Entwicklung des mathematischen und naturwissenschaftlichen Denkens und Wissens in der frühen Kindheit und zieht Schlussfolgerungen für eine darauf aufbauende frühe Bildung in diesen Bereichen. Susanna Jeschoneks Expertisen behandeln die Entwicklung des kindlichen Verständnisses der Bereiche ,Magnetismus“ und ,Akustik“ und geben Empfehlungen für die Aufbereitung von Bildungsangeboten zu diesen Themenschwerpunkten in der Praxis. In der Expertise von Salman Ansari stehen Prozesse des Lehrens und Lernens aus der Sicht der kognitiven Wissenschaften im Mittelpunkt. Ansari geht auf verschiedene Konzepte und Angebote der Stiftung „Haus der kleinen Forscher“ ein, spricht Empfehlungen für die Weiterentwicklung dieser Themen aus und verdeutlicht dies anhand von konkreten Beispielen für die praktische Umsetzung. 
Band 5 (2013)

Yvonne Anders, Ilonca Hardy, Sabina Pauen, Jörg Ramseger, Beate Sodian, Mirjam Steffensky

Der fünfte Band stellt Ziele naturwissenschaftlicher Bildung für Kinder und pädagogische Fach-und Lehrkräfte sowie prozessbezogene Qualitätskriterien für den naturwissenschaftlichen Unterricht im Elementar- und Primarbereich in den Fokus. Yvonne Anders, Ilonca Hardy, Sabina Pauen, Beate Sodian und Mirjam Steffensky spezifizieren in ihren Expertisen pädagogisch-inhaltliche Zieldimensionen naturwissenschaftlicher Bildung im Kita- und Grundschulalter. Neben einer theoretischen Fundierung verschiedener Zielbereiche werden Instrumente für deren Messung aufgeführt. Jörg Ramseger formuliert in seiner Expertise zehn Qualitätskriterien für den naturwissenschaftlichen Unterricht. Diese prozessbezogenen Kriterien können pädagogische Fach- und Lehrkräfte bei der Unterrichtsplanung sowie bei der Selbstevaluation naturwissenschaftlicher Angebote im Elementar- und Primarbereich unterstützen.

\section{Band 6 (2014)}

\section{Yvonne Anders, Itala Ballaschk, Wolfgang Tietze}

Im sechsten Band der Schriftenreihe mit einem Geleitwort von Wolfgang Tietze berichten Yvonne Anders und Itala Ballaschk die Ergebnisse ihrer Studie zur Untersuchung der Reliabilität und Validität des Zertifizierungsverfahrens der Stiftung „Haus der kleinen Forscher“, mit dem sich Bildungseinrichtungen nach bestimmten Qualitätskriterien als „Haus der kleinen Forscher“ zertifizieren lassen können. Insgesamt konnte in der Studie das Potenzial des Verfahrens für die Messung der naturwissenschaftsbezogenen Bildungsqualität in pädagogischen Einrichtungen belegt und Ansatzpunkte für weitere Optimierungen aufgezeigt werden.
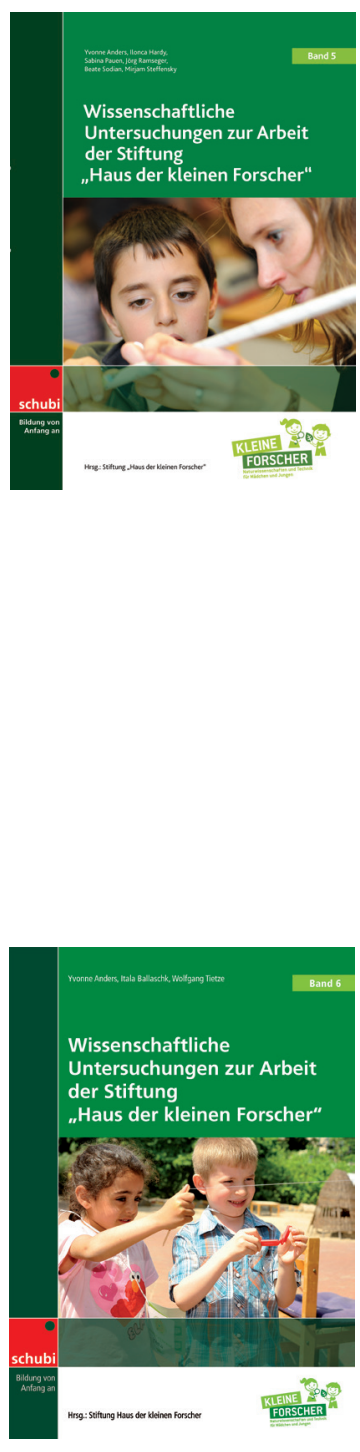


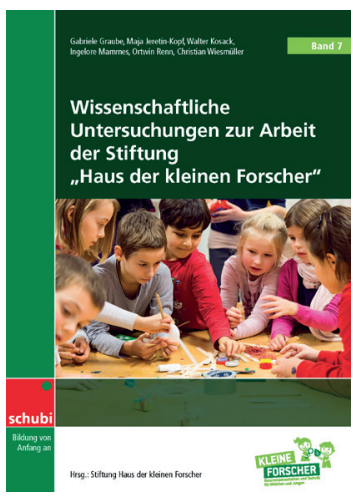

Band 7 (2015)

Gabriele Graube, Maja Jeretin-Kopf, Walter Kosack, Ingelore Mammes, Ortwin Renn, Christian Wiesmüller

Der siebte Band der Reihe mit Geleitwort von Ortwin Renn fokussiert Ziele und Konzepte technischer Bildung im Elementar- und Primarbereich. Walter Kosack, Maja Jeretin-Kopf und Christian Wiesmüller spezifizieren in ihrer Expertise pädagogisch-inhaltliche Zieldimensionen technischer Bildung im Kita- und Grundschulalter. Neben einer theoretischen Fundierung verschiedener Zielbereiche werden Instrumente für deren Messung aufgeführt. Die Autoren stellen in zwei Berichten die Ergebnisse empirischer Studien dar. Zum einen wurde der Einfluss verschiedener technikdidaktischer Materialsysteme auf die kindliche Motivation, problemlösendes Denken und technische Kreativität, und zum anderen der Einfluss verschiedener technikdidaktischer Methoden auf die kindliche Motivation sowie technikspezifische Denk- und Handlungsweisen untersucht. Gabriele Graube und Ingelore Mammes beschreiben in ihrem Beitrag ein didaktisches Konzept zur Unterstützung des professionellen Handelns pädagogischer Fach- und Lehrkräfte bei der Begleitung kindlicher Bildungsprozesse in ihrer Auseinandersetzung mit Natur und Technik.

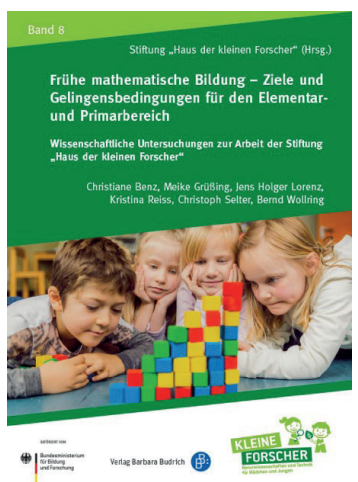

\section{Band 8 (2017)}

Christiane Benz, Meike Grüßing, Jens Holger Lorenz, Kristina Reiss, Christoph Selter, Bernd Wollring

Der achte Band der Reihe mit einem Geleitwort von Kristina Reiss stellt die Ziele und Gelingensbedingungen mathematischer Bildung im Elementar- und Primarbereich in den Fokus. Christiane Benz, Meike Grüßing, Jens Holger Lorenz, Christoph Selter und Bernd Wollring spezifizieren in ihrer Expertise pädagogisch-inhaltliche Zieldimensionen mathematischer Bildung im Kita- und Grundschulalter. Neben einer theoretischen Fundierung verschiedener Zielbereiche werden Instrumente für deren Messung aufgeführt. Des Weiteren erörtern die Autorinnen und Autoren Gelingensbedingungen für eine effektive und wirkungsvolle frühe mathematische Bildung in der Praxis. Sie geben zudem Empfehlungen für die Weiterentwicklung der Stiftungsangebote und die wissenschaftliche Begleitung der Stiftungsarbeit im Bereich Mathematik. Das Schlusskapitel des Bandes beschreibt die Umsetzung dieser fachlichen Empfehlungen in den inhaltlichen Angeboten der Stiftung „Haus der kleinen Forscher“. 
Band 9 (2018)

Nadine Bergner, Peter Hubwieser, Hilde Köster, Johannes Magenheim, Kathrin Müller, Ralf Romeike, Ulrik Schroeder, Carsten Schulte

Der neunte Band mit einem Geleitwort/Streitgespräch von Peter Hubwieser und Johannes Magenheim stellt die Ziele und Gelingensbedingungen informatischer Bildung im Elementar- und Primarbereich in den Fokus. Nadine Bergner, Hilde Köster, Johannes Magenheim, Kathrin Müller, Ralf Romeike, Ulrik Schroeder und Carsten Schulte spezifizieren in ihrer Expertise pädagogisch-inhaltliche Zieldimensionen informatischer Bildung im Kita- und Grundschulalter. Neben einer theoretischen Fundierung verschiedener Zielbereiche erörtern die Autorinnen und Autoren Gelingensbedingungen für eine effektive und wirkungsvolle frühe informatische Bildung in der Praxis. Sie geben zudem Empfehlungen für die Entwicklung der Stiftungsangebote und die wissenschaftliche Begleitung der Stiftungsarbeit im Bereich Informatik. Nadine Bergner und Kathrin Müller beschreiben in ihrer Fachempfehlung eine Auswahl an Informatiksystemen für Kinder im Kita- und Grundschulalter und geben anhand fachlicher Kriterien Empfehlungen für besonders geeignete Informatiksysteme und deren Verwendung im Elementar- und Primarbereich.

Das Schlusskapitel des Bandes beschreibt die Umsetzung dieser fachlichen Empfehlungen in den inhaltlichen Angeboten der Stiftung „Haus der kleinen Forscher“ - mit und ohne Computer.

\section{Band 10 (2018)}

Yvonne Anders, Julia Barenthien, Ilonca Hardy, Andreas Hartinger,

Miriam Leuchter, Elisa Oppermann, Sabina Pauen, Astrid Rank, Hans-Günther Roßbach, Mirjam Steffensky, Päivi Taskinen, Sabrina Tietze, Anja Wildemann, Tobias Ziegler

Der zehnte Band der Reihe mit einem Geleitwort von Hans-Günther Roßbach stellt die Ergebnisse der Studien zu Wirkungen naturwissenschaftlicher Bildungsangebote auf pädagogische Fachkräfte und Kinder im Vorschulalter vor. Mirjam Steffensky, Yvonne Anders, Julia Barenthien, Ilonca Hardy, Miriam Leuchter, Elisa Oppermann, Päivi Taskinen und Tobias Ziegler untersuchten die Wirkungen naturwissenschaftlicher Bildungsangebote auf die kognitiven und motivationalen Kompetenzen von pädagogischen Fachkräften und Kindern in Kitas. Insgesamt konnte aufgezeigt
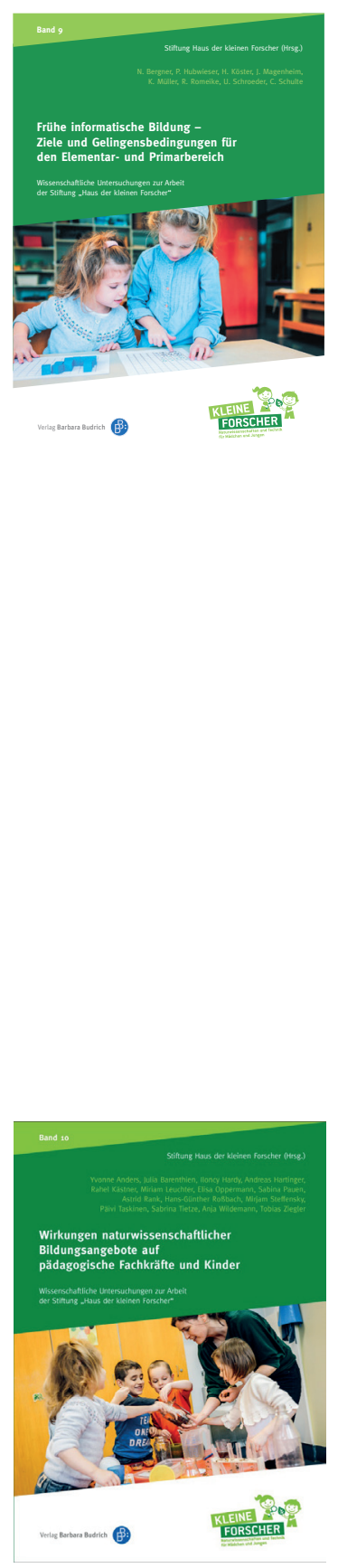
werden, dass die Teilnahme an naturwissenschaftlichen Fortbildungen in einem positiven Zusammenhang mit den naturwissenschaftlichen professionellen Kompetenzen pädagogischer Fachkräfte steht und dass Kinder aus Einrichtungen mit einem expliziten naturwissenschaftlichen Schwerpunkt höhere Lernfreude und höheres Selbstvertrauen in Bezug auf Naturwissenschaften zeigen als Kinder in Einrichtungen ohne naturwissenschaftlichen Schwerpunkt. Astrid Rank, Anja Wildemann, Sabina Pauen, Andreas Hartinger, Sabrina Tietze und Rahel Kästner untersuchten die Interaktionsqualität und mögliche sprachliche Bildungswirkungen im Kontext naturwissenschaftlicher Bildungsangebote bei Vorschulkindern. Die Studie legt nahe, dass naturwissenschaftliche Fortbildungen die naturwissenschaftlichen Kompetenzen wie auch den Spracherwerb der Kinder unterstützen und die Prozessqualität der Fachkräfte in naturwissenschaftsbezogenen Situationen bestimmen können. Das Schlusskapitel des Bandes beschreibt die Umsetzung der wissenschaftlichen Empfehlungen in den inhaltlichen Angeboten der Stiftung „Haus der kleinen Forscher“ und ihrer fachlichen Weiterentwicklung. 


\section{Zieldimensionen für Multiplikatorinnen und Multiplikatoren früher MINT-Bildung}

Im Rahmen der Schriftenreihe „Wissenschaftliche Untersuchungen zur Arbeit der Stiftung ,Haus der kleinen Forscher““ werden regelmäßig wissenschaftliche Beiträge von renommierten Expertinnen und Experten aus dem Bereich der frühen Bildung veröffentlicht. Diese Schriftenreihe dient einem fachlichen Dialog zwischen Stiftung, Wissenschaft und Praxis, mit dem Ziel, allen Kitas, Horten und Grundschulen in Deutschland fundierte Unterstützung für ihren Bildungsauftrag zu geben.

Der elfte Band der Schriftenreihe mit einem Geleitwort von Rudolf Tippelt fokussiert die Zieldimensionen für Multiplikatorinnen und Multiplikatoren früher MINT-Bildung sowie einer frühen Bildung für nachhaltige Entwicklung. Olaf Köller und Esther Winther formulieren, basierend auf den Merkmalen erfolgreicher Lehrkräfte- und Erwachsenenbildung, ein Modell professioneller Kompetenz für Multiplikatorinnen und Multiplikatoren früher MINT-Bildung. Olaf Köller, Johannes Magenheim, Uwe Pfenning, Jörg Ramseger, Mirjam Steffensky, Christian Wiesmüller, Esther Winther und Bernd Wollring erörtern zentrale Zieldimensionen guter MINT-Erwachsenenbildnerinnen und -bildner wie die motivationalen Orientierungen und Überzeugungen, die selbstregulativen Fähigkeiten, die fachspezifischen und fachdidaktischen sowie die pädagogischpsychologischen Zieldimensionen des Professionswissens. Sie geben zudem Empfehlungen für die Priorisierung der Zieldimensionen und für die (Weiter-)Entwicklung der Stiftungsangebote. Heike Molitor kommentiert diese Zieldimensionen aus Sicht einer Bildung für nachhaltige Entwicklung und beschreibt, welchen Anforderungen Multiplikatorinnen und Multiplikatoren im Kontext einer (MINT-)Bildung für nachhaltige Entwicklung begegnen.

Das Fazit des Bandes beschreibt die Umsetzung der fachlichen Empfehlungen in die inhaltlichen Angebote der Stiftung „Haus der kleinen Forscher" und gibt einen Ausblick auf die weitere wissenschaftliche Begleitung der Stiftungsarbeit.

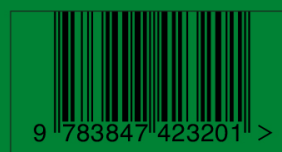

www.budrich-verlag.de

www.haus-der-kleinen-forscher.de 